\title{
Palladium-Catalyzed Tandem Regioselective Oxidative Coupling from Indoles and Maleimides: One-Pot Synthesis of Indolopyrrolocarbazoles and Related Indolylmaleimides
}

Yu-Long An ${ }^{\mathrm{a}}$, Zhen-Hua Yang ${ }^{\mathrm{a}}$, He-Hui Zhang ${ }^{\mathrm{a}}$ and Sheng-Yin Zhao ${ }^{\text {a,b }}$

${ }^{a}$ Department of chemistry, Donghua University, No.2999 North Renmin Road, Shanghai 201620, China;

${ }^{\mathrm{b}}$ State Key Laboratory of Bioorganic \& Natural Products Chemistry, Shanghai Institute of Organic

Chemistry, Chinese Academy of Sciences, Shanghai 200032, China.

Fax: (+) 8621 67792608-805;

E-mail: syzhao8@dhu.edu.cn

\section{Supporting Information}

\section{Contents:}

General information

General procedure for the synthesis of products

Procedure for compounds 3a-k (page 2), 31 (page 8), and 3m (page 8-9)

Procedure for compounds 4a-e (page 9), and 4f-g (page 12)

Procedure for compounds 5a (page 13), 5b-c (page 14-15), 5d-e (page16)

Procedure for compounds 7a-b (page 17)

Procedure for compounds 8a-b, 9a-b (page 18-19)

Procedure for $\mathbf{3 c}, \mathbf{3 g}$ from 9 in the presence of $\mathrm{SeO}_{2}$ (page 21)

Procedure for $\mathbf{3 c}, \mathbf{3 g}$ from $\mathbf{9}$ in the palladium-catalyzed protocol (page 21)

Characterization of compounds

NMR spectra 


\section{General information}

Unless otherwise noted, all the materials were purchased from commercial suppliers and used without further purification. Petroleum ether (PE) refers to a hydrocarbon mixture with a boiling range of $60-90{ }^{\circ} \mathrm{C}$. TLC was performed on silica gel polygram SILG/UV 254 plates and visualized by quenching of UV fluorescence $\left(\lambda_{\max }=254 \mathrm{~nm}\right)$. Silica gel $(100-200$ microns $)$ was used for all chromatographic separations. Melting points were determined with RY-1 apparatus and uncorrected. IR spectra were recorded as $\mathrm{KBr}$ pellets on a Shimadzu model 470 spectrophotometer. NMR $\left({ }^{1} \mathrm{H}\right.$ and ${ }^{13} \mathrm{C}$ ) spectra were recorded using a Bruker AV $400 \mathrm{MHz}$ spectrometer in DMSO- $d_{6}$ and $\mathrm{CDCl}_{3}$ with TMS as internal standard. Chemical shifts $(\delta)$ were recorded in ppm. Mass spectra were acquired on Waters micromass GCT premier, Agilent technologies 5973N and thermo Fisher scientific LTQ FT Ultra.

General procedure for the synthesis of indolo[3,2-a]pyrrolo[3,4-c]carbazole-6,8-diones (3a-k) A mixture of indole 1 (1.5 mmol, 2 equiv), maleimide 2 ( $0.75 \mathrm{mmol}, 1$ equiv), $\mathrm{Pd}(\mathrm{OAc})_{2}(16.8 \mathrm{mg}$, $0.075 \mathrm{mmol}, 0.1$ equiv), $\mathrm{Cu}(\mathrm{OAc})_{2}(204.3 \mathrm{mg}, 1.125 \mathrm{mmol}, 1.5$ equiv) and DMF/DMSO ( $3 \mathrm{~mL}, \mathrm{~V}: \mathrm{V}=$ 1:5) were stirred at $80{ }^{\circ} \mathrm{C}$ for $18 \mathrm{hr}$ (TLC monitoring, acetone-PE, 1:1 4). The mixture was cooled to room temperature before quenched with $\mathrm{H}_{2} \mathrm{O}(3 \mathrm{~mL})$ and extracted with ethyl acetate $(50 \mathrm{~mL})$. Filtering out the insoluble substances, the organic phases were washed with $\mathrm{H}_{2} \mathrm{O}(3 \times 25 \mathrm{~mL})$, and dried $\left(\mathrm{Na}_{2} \mathrm{SO}_{4}\right)$. The solvent was removed and the residue was purified by silica gel column chromatography (eluent: acetone-PE, 1:3 12) to yield the pure products. The products were characterized by IR, ${ }^{1} \mathrm{H}$ NMR, ${ }^{13} \mathrm{C}$ NMR, MS and HRMS analyses.

$5 H$-indolo[3,2-a]pyrrolo[3,4-c]carbazole-6,8(7H,13H)-dione (3a)<smiles>O=c1[nH]c(=O)c2c3[nH]c4ccccc4c3c3[nH]c4ccccc4[nH]c3c12</smiles>

Yield $182 \mathrm{mg}(75 \%)$ as yellow solid; Mp: >350 ${ }^{\circ} \mathrm{C}$. IR (KBr): 3308, 1732, 1690, 1649, 1607, 1463, 1373, 1328, 1235, $733 \mathrm{~cm}^{-1} .{ }^{1}$ H NMR (400 MHz, DMSO-d $\left.\boldsymbol{d}_{6}\right): \delta 7.63-7.22(\mathrm{~m}, 4 \mathrm{H}), 7.75$ (s, 2H), 8.79 
(s, 1H) , 8.95 (s, 1H), $11.06(\mathrm{~s}, 1 \mathrm{H}), 12.19(\mathrm{~s}, 1 \mathrm{H}), 12.32$ (s, 1H); ${ }^{13}$ C NMR (101 MHz, DMSO-d $)_{\text {) }} \delta$ $107.52,110.88,111.60,111.96,112.30,120.15,120.20,120.39,121.31,121.85,123.80,124.76$, 126.27, 126.32, 132.54, 135.32, 138.31, 141.15, 170.30, 171.10; MS (ESI): $\mathrm{m} / \mathrm{z}=326.1[\mathrm{M}+\mathrm{H}]^{+}$, HRMS (ESI): $\mathrm{m} / \mathrm{z}$ calcd for $\mathrm{C}_{20} \mathrm{H}_{12} \mathrm{~N}_{3} \mathrm{O}_{2}: 326.0924$, found 326.0920 .

2,10-dimethoxy-5H-indolo[3,2-a]pyrrolo[3,4-c]carbazole-6,8(7H,13H)-dione (3b)<smiles>COc1ccc2c(c1)-c1[nH]c3c(c4c([nH]c5ccc(OC)cc54)c-3c3c1C(=O)NC3=O)N2</smiles>

Yield $233 \mathrm{mg}(81 \%)$ as red brown solid; Mp: 325-328 ${ }^{\circ} \mathrm{C}$. IR (KBr): 3355, 3304, 2920, 2843, 1730, 1690, 1636, 1476, 1280, 1216, 1027, 864, $621 \mathrm{~cm}^{-1} .{ }^{1} \mathbf{H}$ NMR (400 MHz, DMSO-d 6 ): $\delta 3.91$ (s, 3H), $3.98(\mathrm{~s}, 3 \mathrm{H}), 7.18(\mathrm{~d}, J=8.6 \mathrm{~Hz}, 2 \mathrm{H}), 7.64(\mathrm{~d}, J=8.5 \mathrm{~Hz}, 2 \mathrm{H}), 8.27(\mathrm{~s}, 1 \mathrm{H}), 8.55(\mathrm{~d}, J=13.1 \mathrm{~Hz}, 1 \mathrm{H})$, 10.99 (s, 1H), 11.97 (s, 1H), 12.12 (s, 1H); ${ }^{13}$ C NMR (101 MHz, DMSO-d (1) $^{\delta} \delta$ 56.03, 56.61, 104.96, $107.25,107.68,111.19,111.99,112.41,113.31,115.21,115.95,121.00,122.40,125.18,133.30$, 136.10, 136.31, 139.31, 154.45, 154.75, 170.72, 171.59; MS (ESI): m/z=386.1[M+H] ${ }^{+}$, HRMS (ESI): $\mathrm{m} / \mathrm{z}$ calcd for $\mathrm{C}_{22} \mathrm{H}_{16} \mathrm{~N}_{3} \mathrm{O}_{4}: 386.1135$, found 386.1132 .

5, 13-dimethyl-5H-indolo[3,2-a]pyrrolo[3,4-c]carbazole-6,8(7H,13H)-dione (3c)<smiles></smiles>

Yield $152 \mathrm{mg}(60 \%)$ as red brown solid; Mp: 294-296 ${ }^{\circ} \mathrm{C}$. IR (KBr): 3455, 3385, 2923, 2850, 1783 , 1716, 1668, 1543, 1460, 1376, 1309, 1229, 970, $736 \mathrm{~cm}^{-1} .{ }^{\mathbf{1}}$ H NMR (400 MHz, DMSO-d $\boldsymbol{d}_{\boldsymbol{6}}$ : $\delta 4.12$ (s, 3H), $4.30(\mathrm{~s}, 3 \mathrm{H}), 7.32(\mathrm{~s}, 2 \mathrm{H}), 7.57(\mathrm{~d}, J=15.7 \mathrm{~Hz}, 4 \mathrm{H}), 8.40(\mathrm{~d}, J=7.0 \mathrm{~Hz}, 1 \mathrm{H}), 9.00(\mathrm{~d}, J=6.9 \mathrm{~Hz}$, 1H),11.02 (s, 1H); ${ }^{13}$ C NMR (101 MHz, DMSO-d $\left.{ }_{6}\right): \delta 34.28,35.74,107.74,110.20,110.58,111.04$, $113.01,119.97,120.72,121.04,121.47,123.72,124.69,126.55,126.68,126.87,136.63,141.60$, 
142.05, 143.12, 169.63,170.37; MS (ESI): $\mathrm{m} / \mathrm{z}=354.1[\mathrm{M}+\mathrm{H}]^{+}$, HRMS (ESI): $\mathrm{m} / \mathrm{z}$ calcd for $\mathrm{C}_{22} \mathrm{H}_{16} \mathrm{~N}_{3} \mathrm{O}_{2}: 354.1237$, found 354.1233.

2,10-dichloro-5H-indolo[3,2-a]pyrrolo[3,4-c]carbazole-6,8(7H,13H)-dione (3d)<smiles>O=C1NC(=O)c2c1c1[nH]c3ccc(Cl)cc3c1c1[nH]c3ccc(Cl)cc3c21</smiles>

Yield $162 \mathrm{mg}(55 \%)$ as red brown solid; Mp: >350 ${ }^{\circ} \mathrm{C}$. IR (KBr): 3468, 3404, 1790, 1719, 1687, 1642, 1511, 1453, 1312, 1264, $790 \mathrm{~cm}^{-1} .{ }^{1} \mathbf{H}$ NMR (400 MHz, DMSO-d $\left.)_{6}\right): \delta .51(\mathrm{t}, J=8.7 \mathrm{~Hz}, 2 \mathrm{H})$, $7.70(\mathrm{t}, J=8.7 \mathrm{~Hz}, 2 \mathrm{H}), 8.86(\mathrm{~d}, J=19.7 \mathrm{~Hz}, 2 \mathrm{H}), 11.13(\mathrm{~s}, 1 \mathrm{H}), 12.30(\mathrm{~s}, 1 \mathrm{H}), 12.67(\mathrm{~s}, 1 \mathrm{H}) ;{ }^{13} \mathbf{C}$ NMR (101 MHz, DMSO-d $\left.\boldsymbol{d}_{6}\right): \delta 107.88,109.81,110.55,112.92,113.47,120.98,121.02,122.18$, $122.54,124.32,124.69,125.51,125.76,125.96,132.99,138.39,139.35(2 \mathrm{C}), 169.79,170.72$; MS (ESI): $\mathrm{m} / \mathrm{z}=394.0[\mathrm{M}+\mathrm{H}]^{+}$, HRMS (ESI): $\mathrm{m} / \mathrm{z}$ calcd for $\mathrm{C}_{20} \mathrm{H}_{10} \mathrm{Cl}_{2} \mathrm{~N}_{3} \mathrm{O}_{2}: 394.0145$, found 394.0144. 2,10-dimethoxy-7-methyl-5H-indolo[3,2-a]pyrrolo[3,4-c]carbazole-6,8(7H,13H)-dione (3e)

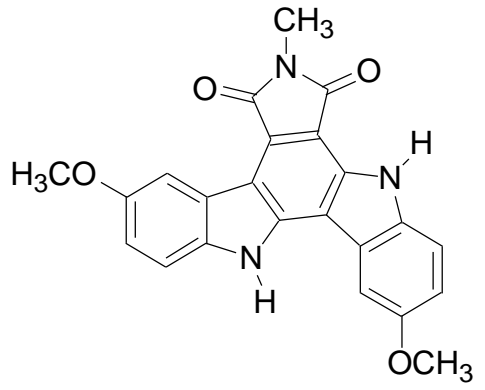

Yield $245 \mathrm{mg}$ (82\%) as red brown solid; Mp: 310-313 ${ }^{\circ} \mathrm{C}$. IR (KBr): 3378, 3330, 2923, 2846, 1745,

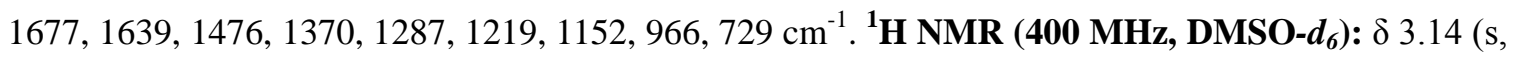
3H), $3.90(\mathrm{~s}, 3 \mathrm{H}), 3.98(\mathrm{~s}, 3 \mathrm{H}), 7.16(\mathrm{td}, J=8.6,1.8 \mathrm{~Hz}, 2 \mathrm{H}), 7.62(\mathrm{dd}, J=13.4,8.8 \mathrm{~Hz}, 2 \mathrm{H}), 8.52(\mathrm{~s}$, 1H), $8.23(\mathrm{~s}, 1 \mathrm{H}), 11.93$ (s, 1H),12.07 (s, 1H); ${ }^{13}$ C NMR (101 MHz, DMSO-d 6 ): $\delta$ 23.43, 55.33, $56.03,104.41,105.98,106.19,110.50,111.63,111.87,112.69,114.95,115.37,120.44,121.66,123.59$, 132.71, 135.56, 135.76, 138.58, 153.87, 154.17, 168.75, 169.62; MS (ESI): $\mathrm{m} / \mathrm{z}=400.1[\mathrm{M}+\mathrm{H}]^{+}$, HRMS (ESI): $\mathrm{m} / \mathrm{z}$ calcd for $\mathrm{C}_{23} \mathrm{H}_{18} \mathrm{~N}_{3} \mathrm{O}_{2}: 400.1292$, found 400.1289 . 
7-methyl-5H-indolo[3,2-a]pyrrolo[3,4-c]carbazole-6,8(7H,13H)-dione (3f)

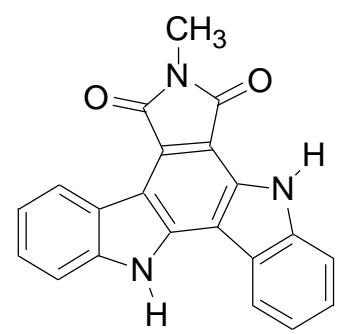

Yield $185 \mathrm{mg}(73 \%)$ as yellow solid; Mp: $>350{ }^{\circ} \mathrm{C}$. IR (KBr): 3461, 3356, 2923, 2850, 1780, 1745, 1687, 1645, 1604, 1553, 1453, 1421, 1367, 1328, 1226, 986, 941, 867, $739 \mathrm{~cm}^{-1} .{ }^{\mathbf{1}}$ H NMR (400 MHz, DMSO-d $\left.\boldsymbol{d}_{6}\right): \delta 3.15(\mathrm{~s}, 3 \mathrm{H}), 7.33(\mathrm{t}, J=6.9 \mathrm{~Hz}, 1 \mathrm{H}), 7.47-7.37(\mathrm{~m}, 1 \mathrm{H}), 7.53(\mathrm{~d}, J=7.8 \mathrm{~Hz}, 2 \mathrm{H})$, $7.74(\mathrm{dd}, J=17.4,7.8 \mathrm{~Hz}, 2 \mathrm{H}), 8.76(\mathrm{~d}, J=7.4 \mathrm{~Hz}, 1 \mathrm{H}), 8.92(\mathrm{~d}, J=7.4 \mathrm{~Hz}, 1 \mathrm{H}), 12.15(\mathrm{~s}, 1 \mathrm{H}), 12.28$ (s, 1H); ${ }^{13}$ C NMR (101 MHz, DMSO-d d $_{\text {) }} \delta$ 24.04, 106.83, 111.16, 112.00, 112.52, 112.67, 120.55, $120.63,120.78,121.62,122.28,124.09,124.19,126.68,126.79,132.95,138.61,141.59,141.64$, 169.32, 170.05; MS (ESI): $\mathrm{m} / \mathrm{z}=340.1[\mathrm{M}+\mathrm{H}]^{+}$, HRMS (ESI): $\mathrm{m} / \mathrm{z}$ calcd for $\mathrm{C}_{21} \mathrm{H}_{14} \mathrm{~N}_{3} \mathrm{O}_{2}: 340.1081$, found 340.1080 .

7-phenyl-5H-indolo[3,2-a]pyrrolo[3,4-c]carbazole-6,8(7H,13H)-dione (3g)

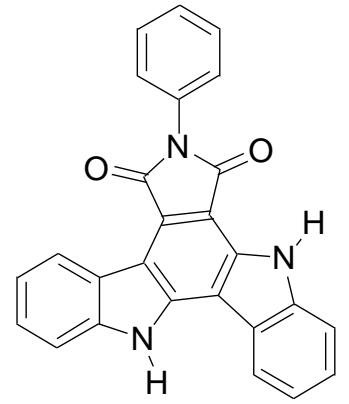

Yield $240 \mathrm{mg}(80 \%)$ as yellow solid; Mp: >350 ${ }^{\circ} \mathrm{C}$. IR (KBr): 3433, 3366, 1744, 1728, 1686, 1648, 1546, 1458, 1369, 1252, 975, 854, $728 \mathrm{~cm}^{-1} .{ }^{1}$ H NMR (400 MHz, DMSO-d 6 ): $\delta 7.54(\mathrm{~m}, 11 \mathrm{H}), 8.80$ (s, 1H), 8.95 (s, 1H), 12.28 (s, 1H), 12.39 (s, 1H); ${ }^{13}$ C NMR (101 MHz, DMSO-d N $^{2} \delta$ 106.61, 111.58, $112.14,112.76,112.83,120.65,120.72,120.95,121.64,122.38,123.76,124.24,126.90,126.99$, 128.01(2C), 128.08, 129.32(2C), 132.98, $133.23,138.95,141.74,141.76,168.20,169.00$; MS (ESI): $\mathrm{m} / \mathrm{z}=402.1[\mathrm{M}+\mathrm{H}]^{+}, \mathbf{H R M S}(\mathbf{E S I}): \mathrm{m} / \mathrm{z}$ calcd for $\mathrm{C}_{26} \mathrm{H}_{16} \mathrm{~N}_{3} \mathrm{O}_{2}: 402.1237$, found 402.1231 . 2,10-dimethoxy-7-phenyl-5H-indolo[3,2-a]pyrrolo[3,4-c]carbazole-6,8(7H,13H)-dione (3h) 


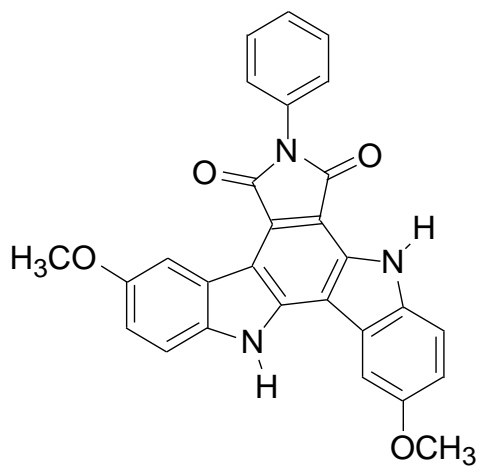

Yield $293 \mathrm{mg}$ (85\%) as yellow brown solid; Mp: 300-303 'C. IR (KBr): 3397, 2918, 2853, 1777, 1707, 1594, 1485, 1447, 1373, 1279, 1198, 961, 890, $746 \mathrm{~cm}^{-1} .{ }^{1}$ H NMR (400 MHz, DMSO-d 6 ): $\delta$ $3.88(\mathrm{~s}, 3 \mathrm{H}), 3.99(\mathrm{~s}, 3 \mathrm{H}), 7.19(\mathrm{t}, J=8.4 \mathrm{~Hz}, 2 \mathrm{H}), 7.49(\mathrm{~s}, 1 \mathrm{H}), 7.64(\mathrm{~m}, 6 \mathrm{H}), 8.28(\mathrm{~s}, 1 \mathrm{H}), 8.54(\mathrm{~s}$, 1H) 12.07 (s, 1H), 12.19 (s, 1H); ${ }^{13}$ C NMR (101 MHz, DMSO-d d $^{2}$ : $\delta$ 55.91, 56.64, 105.01, 106.33, $106.74,111.47,112.43,112.58,113.41,115.81,121.02,122.24,123.70,128.10(2 \mathrm{C}), 129.26$, 129.31(2C), 132.97, 133.50, 136.22, 136.47, 139.45, 154.53, 154.84, 168.19, 169.15; MS (ESI): m/z $=462.1[\mathrm{M}+\mathrm{H}]^{+}$, HRMS $\left(\right.$ESI): $\mathrm{m} / \mathrm{z}$ calcd for $\mathrm{C}_{28} \mathrm{H}_{20} \mathrm{~N}_{3} \mathrm{O}_{4}: 462.1448$, found 462.1448 .

2,10-bis(benzyloxy)-7-phenyl-5H-indolo[3,2-a]pyrrolo[3,4-c]carbazole-6,8 $(7 H, 13 H)$-dione (3i)

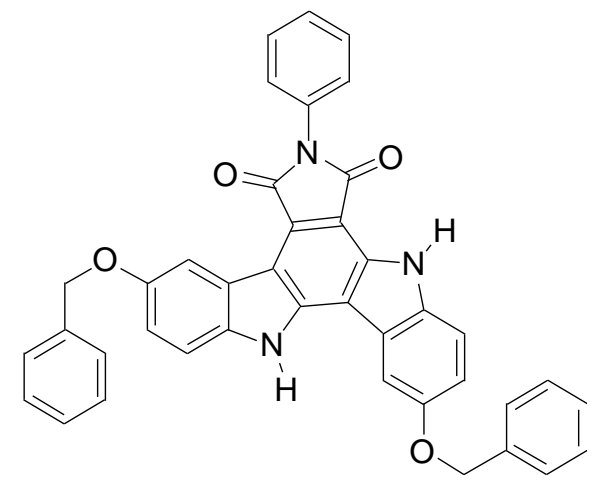

Yield $358 \mathrm{mg}$ (78\%) as red brown solid; Mp: 268-270 ${ }^{\circ} \mathrm{C}$. IR (KBr): 3385, 2917, 2853, 1745, 1697, 1642, 1588, 1482, 1367, 1280, 1219, 1117, 954, 861, 736, $697 \mathrm{~cm}^{-1} .{ }^{1} \mathbf{H}$ NMR (400 MHz, DMSO-d $)$ : $\delta 5.19(\mathrm{~s}, 2 \mathrm{H}), 5.29(\mathrm{~s}, 2 \mathrm{H}), 7.99-7.11(\mathrm{~m}, 19 \mathrm{H}), 8.41(\mathrm{~s}, 1 \mathrm{H}), 8.63(\mathrm{~s}, 1 \mathrm{H}), 12.07(\mathrm{~s}, 1 \mathrm{H}), 12.16(\mathrm{~s}$, 1H); ${ }^{13}$ C NMR (101 MHz, DMSO-d $\left.\boldsymbol{d}_{\boldsymbol{\sigma}}\right): \delta$ 70.38, 71.03, 106.36, 106.55, 108.32, 111.41, 112.39, $112.56,113.38,116.33,116.36,116.47,121.08,122.24,123.78,128.10(2 \mathrm{C}), 128.27,128.35$ (2C), $128.43,128.60$ (2C), 128.88 (2C), 128.97 (2C), 129.33 (2C), 133.00, 133.60, 136.42, 136.67, 137.79, 
137.84, 139.48, 153.58, 153.94, 168.18, 169.12; MS (ESI): $\mathrm{m} / \mathrm{z}=614.2[\mathrm{M}+\mathrm{H}]^{+}$, HRMS (ESI): m/z calcd for $\mathrm{C}_{40} \mathrm{H}_{28} \mathrm{~N}_{3} \mathrm{O}_{4}: 614.2074$, found 614.2066.

\section{4,12-dimethyl-7-phenyl-5H-indolo[3,2-a]pyrrolo[3,4-c]carbazole-6,8(7H,13H)-dione $(3 \mathbf{j})$}

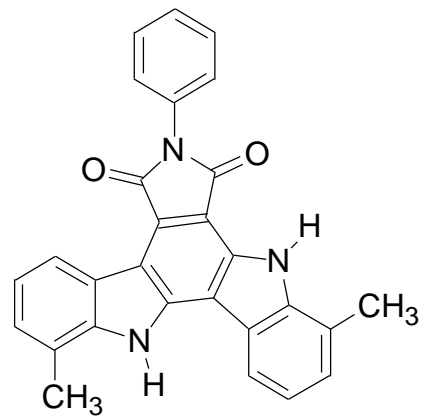

Yield $267 \mathrm{mg}$ (83\%) as yellow brown solid; Mp: 310-312 ${ }^{\circ} \mathrm{C}$. IR (KBr): 3458, 3404, 2911, 2850, 1745, 1687, 1642, 1553, 1488, 1364, 1229, 1152, 986, 886, 739, $697 \mathrm{~cm}^{-1} .{ }^{1}$ H NMR (400 MHz, DMSO-d $\left.\boldsymbol{d}_{6}\right): \delta 2.73(\mathrm{~s}, 3 \mathrm{H}), 2.77(\mathrm{~s}, 3 \mathrm{H}), 7.25-7.36(\mathrm{~m}, 4 \mathrm{H}), 7.47(\mathrm{~s}, 1 \mathrm{H}), 7.60(\mathrm{~s}, 4 \mathrm{H}), 8.87(\mathrm{~d}, J=6.2$ $\mathrm{Hz}, 2 \mathrm{H}), 11.47$ (s, 1H), $11.72(\mathrm{~s}, 1 \mathrm{H}) ;{ }^{13}$ C NMR (101 MHz, DMSO-d $\left.\boldsymbol{d}_{\boldsymbol{6}}\right): \delta 18.13$ (2C), 107.13, 112.73, $113.85,120.71,120.75,120.95,121.20,121.71,121.80,121.85,122.16,123.58,127.89$ (2C), 127.97, 128.01, 128.06, 129.28 (2C), 132.99, 133.64, 139.19, 140.85, 140.88, 167.80, 168.85; MS (ESI): m/z $=430.2[\mathrm{M}+\mathrm{H}]^{+}$, HRMS (ESI): $\mathrm{m} / \mathrm{z}$ calcd for $\mathrm{C}_{28} \mathrm{H}_{20} \mathrm{~N}_{3} \mathrm{O}_{2}: 430.1550$, found 430.1545 .

\section{7-benzyl-5H-indolo[3,2-a]pyrrolo[3,4-c]carbazole-6,8(7H,13H)-dione (3k)}

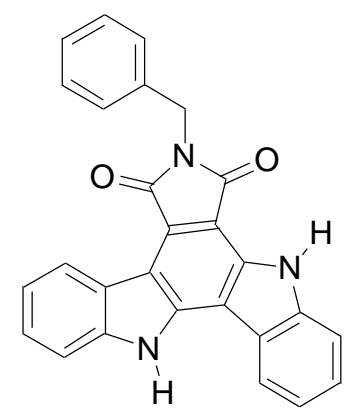

Yield 196 mg (63\%) as brown solid; Mp: 270-272 ${ }^{\circ} \mathrm{C} . \mathbf{I R}(\mathrm{KBr}): 3407,3359,2927,2853,1741,1703$, 1642, 1549, 1508, 1453, 1376, 1351, 1226, 1146, 976, 880, 742, 697, $630 \mathrm{~cm}^{-1} .{ }^{\mathbf{1}} \mathbf{H}$ NMR (400 MHz, DMSO-d $\left.\boldsymbol{d}_{\boldsymbol{\sigma}}\right): \delta 4.91(\mathrm{~s}, 2 \mathrm{H}), 7.48-7.23(\mathrm{~m}, 7 \mathrm{H}), 7.55(\mathrm{q}, J=7.5 \mathrm{~Hz}, 2 \mathrm{H}), 7.83-7.66(\mathrm{~m}, 2 \mathrm{H}), 8.79(\mathrm{~d}$, $J=7.9 \mathrm{~Hz}, 1 \mathrm{H}), 8.93(\mathrm{~d}, J=7.9 \mathrm{~Hz}, 1 \mathrm{H}), 12.24(\mathrm{~s}, 1 \mathrm{H}), 12.36(\mathrm{~s}, 1 \mathrm{H}) ;{ }^{13} \mathbf{C}$ NMR (101 MHz, DMSO-d $\left.\boldsymbol{d}_{6}\right): \delta 40.57,99.41,105.98,110.85,111.53,112.15,112.17,120.04,120.10,120.33,120.98$, $121.78,123.29,123.57,126.30,126.42,127.22,127.31(2 \mathrm{C}), 128.52(2 \mathrm{C}), 132.55,137.31,138.25$, 
141.09, 141.15, 168.41, 169.19; MS (ESI): m/z $=416.1[\mathrm{M}+\mathrm{H}]^{+}$, HRMS (ESI): m/z calcd for $\mathrm{C}_{27} \mathrm{H}_{18} \mathrm{~N}_{3} \mathrm{O}_{2}:$ 416.1394, found 416.1390 .

\section{2-methoxy-5H-indolo[3,2-a]pyrrolo[3,4-c]carbazole-6,8(7H,13H)-dione (3I)}

A mixture of 5-methoxyindole $\mathbf{1 b}(161.9 \mathrm{mg}, 1.1 \mathrm{mmol}, 1.1$ equiv), 3-(1H-indol-3-yl)-succinimide $\mathbf{6 a}$ (224.2 mg, $1.0 \mathrm{mmol}, 1$ equiv), $\mathrm{Pd}(\mathrm{OAc})_{2}\left(22.4 \mathrm{mg}, 0.1 \mathrm{mmol}, 0.1\right.$ equiv), $\mathrm{Cu}(\mathrm{OAc})_{2}(272.4 \mathrm{mg}, 1.5$ mmol, 1.5 equiv) and DMF/DMSO ( $3 \mathrm{~mL}, \mathrm{~V}: \mathrm{V}=1: 5)$ were stirred at $80{ }^{\circ} \mathrm{C}$ for $18 \mathrm{hr}$. The mixture was cooled to room temperature before quenched with $\mathrm{H}_{2} \mathrm{O}(3 \mathrm{~mL})$ and extracted with ethyl acetate (50 mL). Filtering out the insoluble subatances, the organic phases were washed with $\mathrm{H}_{2} \mathrm{O}(3 \times 25$ $\mathrm{mL})$, and dried $\left(\mathrm{Na}_{2} \mathrm{SO}_{4}\right)$. The solvent was removed and the residue was purified by silica gel column chromatography (eluent: acetone-PE, 1:5 4) to yield the pure products $\mathbf{3 l}$ and $\mathbf{5 c}$.<smiles>COc1ccc2[nH]c3c4c(c5c6ccccc6[nH]c5c3c2c1)C(=O)NC4=O</smiles>

Yield $124 \mathrm{mg}(35 \%)$ as saffron yellow solid; Mp: >350 ${ }^{\circ} \mathrm{C}$. IR (KBr): 3466, 3234, 2925, 2853, 1700, 1623, 1572, 1490, 1331, 972, 868, $745 \mathrm{~cm}^{-1} .{ }^{1}$ H NMR (400 MHz, DMSO-d $\boldsymbol{d}_{\text {) }} \delta 3.91$ (s, 3H), 7.21 $(\mathrm{dd}, J=8.8,2.6 \mathrm{~Hz}, 1 \mathrm{H}), 7.35(\mathrm{t}, J=8 \mathrm{~Hz}, 1 \mathrm{H}), 7.55(\mathrm{t}, J=8 \mathrm{~Hz}, 1 \mathrm{H}), 7.74(\mathrm{~d}, J=8.8 \mathrm{~Hz}, 1 \mathrm{H}), 7.81$ $(\mathrm{d}, J=8.2 \mathrm{~Hz}, 1 \mathrm{H}), 8.58(\mathrm{~s}, 1 \mathrm{H}), 8.99(\mathrm{~d}, J=7.9 \mathrm{~Hz}, 1 \mathrm{H}), 10.98(\mathrm{~s}, 1 \mathrm{H}), 11.56(\mathrm{~s}, 1 \mathrm{H}), 11.74(\mathrm{~s}, 1 \mathrm{H})$; ${ }^{13}$ C NMR (101 MHz, DMSO-d $\left.\boldsymbol{d}_{6}\right): \delta 56.00,107.08,112.49,113.25,115.74,116.03,116.50,120.01$, $120.44,120.69,122.11,122.56,124.71,127.11,129.53,130.10,135.63,140.66,154.32,171.81$, 171.86; MS (EI): $\mathrm{m} / \mathrm{z}=355[\mathrm{M}]^{+}, 340,312,241,212,207,143,141,88$. HRMS (EI): m/z calcd for $\mathrm{C}_{21} \mathrm{H}_{13} \mathrm{~N}_{3} \mathrm{O}_{3}: 355.0957$, found: 355.0960 .

\section{2-methoxy-7-phenyl-5H-indolo[3,2-a]pyrrolo[3,4-c]carbazole-6,8(7H,13H)-dione (3m)}

A mixture of 5-methoxyindole $\mathbf{1 b}(161.9 \mathrm{mg}, 1.1 \mathrm{mmol}, 1.1$ equiv), $N$-phenyl-3-(1H-indol-3-yl) -maleimide $4 \mathbf{g}$ (288.3 mg, $1.0 \mathrm{mmol}, 1$ equiv), $\mathrm{Pd}(\mathrm{OAc})_{2}$ (22.4 mg, $0.1 \mathrm{mmol}, 0.1$ equiv), $\mathrm{Cu}(\mathrm{OAc})_{2}$ (272.4 mg, $1.5 \mathrm{mmol}, 1.5$ equiv) DMF/DMSO (3 mL, V:V = 1:5) were stirred at $80{ }^{\circ} \mathrm{C}$ for $18 \mathrm{hr}$. The 
mixture was cooled to room temperature before quenched with $\mathrm{H}_{2} \mathrm{O}(3 \mathrm{~mL})$ and extracted with ethyl acetate $(50 \mathrm{~mL})$. Filtering out the insolubes, the organic phases were washed with $\mathrm{H}_{2} \mathrm{O}(3 \times 25 \mathrm{~mL})$, and dried $\left(\mathrm{Na}_{2} \mathrm{SO}_{4}\right)$. The solvent was removed and the residue was purified by silica gel column chromatography (eluent: acetone-PE, 1:6 5) to yield the pure product $\mathbf{3 m}$ and $\mathbf{5 e}$.

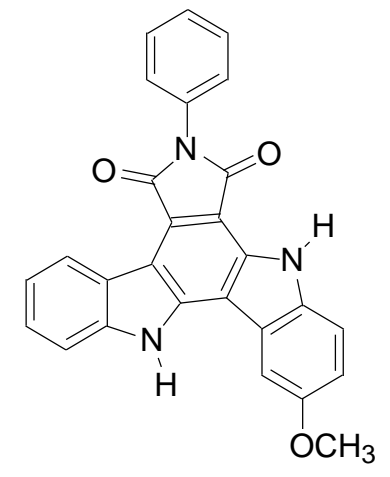

Yield $138 \mathrm{mg}(32 \%)$ as yellow brown solid; Mp: 180-182 ${ }^{\circ} \mathrm{C}$. IR (KBr): 3393, 2920, 1738, 1690, 1629, 1562, 1486, 1365, 866, $748 \mathrm{~cm}^{-1} .{ }^{1}$ H NMR (400 MHz, DMSO-d $\left.\boldsymbol{d}_{\boldsymbol{6}}\right): \delta 3.89$ (s, 3H), 7.22 (d, $J=$ $8.8 \mathrm{~Hz}, 1 \mathrm{H}), 7.36(\mathrm{dd}, J=14.7,7.1 \mathrm{~Hz}, 1 \mathrm{H}), 7.49(\mathrm{dd}, J=12.0,8.2 \mathrm{~Hz}, 1 \mathrm{H}), 7.58(\mathrm{dd}, J=14.0,6.4 \mathrm{~Hz}$, 5H), $7.76(\mathrm{~d}, J=8.8 \mathrm{~Hz}, 1 \mathrm{H}), 7.83(\mathrm{~d}, J=8.1 \mathrm{~Hz}, 1 \mathrm{H}), 8.59(\mathrm{~s}, 1 \mathrm{H}), 9.00(\mathrm{~d}, J=7.9 \mathrm{~Hz}, 1 \mathrm{H}), 11.65(\mathrm{~s}$, 1H),11.83 (s, 1H); ${ }^{13}$ C NMR (101 MHz, DMSO-d 6 ): $\delta$ 55.90, 106.57, 112.61, 113.43, 115.96, 116.30, $117.04,118.67,119.07,120.83,122.02,122.43,124.67,127.32,128.05,128.18(2 \mathrm{C}), 129.25(2 \mathrm{C})$, 129.76, 130.25, 133.07, 135.73, 140.81, 154,43, 169.27, 169.42; MS (EI): $\mathrm{m} / \mathrm{z}=431[\mathrm{M}]^{+}, 415,399$, 287, 252, 241, 212, 153. HRMS (EI): m/z calcd for $\mathrm{C}_{27} \mathrm{H}_{17} \mathrm{~N}_{3} \mathrm{O}_{2}: 431.1270$, found: 431.1265.

\section{General procedure for the synthesis of $4 a-e$}

A mixture of indole 1 ( $0.75 \mathrm{mmol}, 1$ equiv), maleimide 2 ( $0.75 \mathrm{mmol}, 1$ equiv), $\mathrm{Pd}(\mathrm{OAc})_{2}(16.8 \mathrm{mg}$, $0.075 \mathrm{mmol}, 0.1$ equiv), $\mathrm{Cu}(\mathrm{OAc})_{2}(204.3 \mathrm{mg}, 1.125 \mathrm{mmol}, 1.5$ equiv) and DMF/DMSO (3 mL, V:V = 1:5) were stirred at $80{ }^{\circ} \mathrm{C}$ for $18 \mathrm{hr}$ (TLC monitoring, acetone-PE, 1:1 4). The mixture was cooled to room temperature before quenched with $\mathrm{H}_{2} \mathrm{O}(3 \mathrm{~mL})$ and extracted with ethyl acetate $(50 \mathrm{~mL})$. Filtering out the insoluble substances, the organic phases were washed with $\mathrm{H}_{2} \mathrm{O}(3 \times 25 \mathrm{~mL})$, and dried $\left(\mathrm{Na}_{2} \mathrm{SO}_{4}\right)$. The solvent was removed and the residue was purified by silica gel column chromatography (eluent: acetone-PE, 1:4 13) to yield the pure product. The products were characterized by IR, ${ }^{1} \mathrm{H}$ NMR, ${ }^{13} \mathrm{C}$ NMR, MS and HRMS analyses. 
3-(2-methyl-1H-indol-3-yl)-1H-pyrrole-2,5-dione (4a)

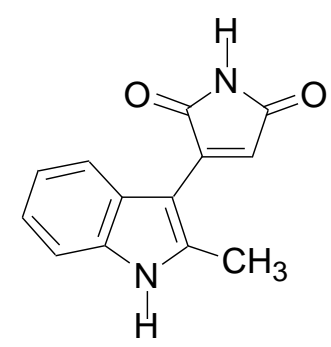

Yield $144 \mathrm{mg}(85 \%)$ as red brown solid; Mp: 228-230 ${ }^{\circ} \mathrm{C}$. IR (KBr): 3436, 3385, 2914, 1758, 1702, 1588, 1450, 1418, 1345, 1232, 966, $742 \mathrm{~cm}^{-1} .{ }^{1}$ H NMR (400 MHz, DMSO-d 6 ): $\delta 2.54$ (s, 3H), $6.58(\mathrm{~s}$, 1H),7.09 (dt, $J=21.4,7.2 \mathrm{~Hz}, 2 \mathrm{H}), 7.34(\mathrm{~d}, J=7.8 \mathrm{~Hz}, 1 \mathrm{H}), 7.71(\mathrm{~d}, J=7.8 \mathrm{~Hz}, 1 \mathrm{H}), 10.79(\mathrm{~s}$, 1H),11.76 (s, 1H); ${ }^{13}$ C NMR (101 MHz, DMSO-d $\left.\boldsymbol{d}_{\boldsymbol{\sigma}}\right): \delta 14.37,102.95,111.48,120.30,120.77,121.97$, 122.12, 127.18, 136.03, 140.36, 142.58, 172.87, 173.17; MS (EI): $\mathrm{m} / \mathrm{z}=226[\mathrm{M}]^{+}, 197,155,154,77$. HRMS (EI): $\mathrm{m} / \mathrm{z}$ calcd for $\mathrm{C}_{13} \mathrm{H}_{10} \mathrm{~N}_{2} \mathrm{O}_{2}: 226.0742$, found: 226.0750 .

3-(2,5-dioxo-2,5-dihydro-1H-pyrrol-3-yl)-1H-indole-2-carboxylic acid ethyl ester (4b)

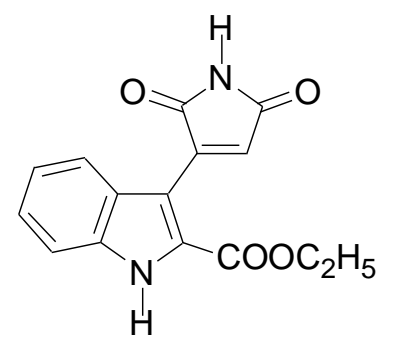

Yield 164 mg (77\%) as yellow solid; Mp: 248-250 ${ }^{\circ} \mathrm{C}$. IR (KBr): 3308, 2981, 1767, 1707, 1629, 1517, 1456, 1332, 1252, 864, $745 \mathrm{~cm}^{-1} .{ }^{1} \mathbf{H}$ NMR (400 MHz, DMSO-d $)^{\text {) }}: \delta 1.26(\mathrm{t}, J=7.1 \mathrm{~Hz}, 3 \mathrm{H}), 4.25$ (q, $J=7.1 \mathrm{~Hz}, 2 \mathrm{H}), 6.97(\mathrm{~s}, 1 \mathrm{H}), 7.20(\mathrm{t}, J=7.5 \mathrm{~Hz}, 1 \mathrm{H}), 7.36(\mathrm{t}, J=7.6 \mathrm{~Hz}, 1 \mathrm{H}), 7.53(\mathrm{~d}, J=8.3 \mathrm{~Hz}$, 1H), $7.67(\mathrm{~d}, J=8.1 \mathrm{~Hz}, 1 \mathrm{H}), 11.04(\mathrm{~s}, 1 \mathrm{H}), 12.51(\mathrm{~s}, 1 \mathrm{H}) ;{ }^{13}$ C NMR (101 MHz, DMSO-d $\left.)\right): \delta 14.31$, $61.42,108.36,113.33,120.95,121.88,125.74,126.50,127.33,136.73,141.36,161.45,171.70$, 172.69; MS (EI): m/z $=284[\mathrm{M}]^{+}, 239,238,167,139,88$. HRMS (EI): m/z calcd for $\mathrm{C}_{15} \mathrm{H}_{12} \mathrm{~N}_{2} \mathrm{O}_{4}$ : 284.0797, found: 284.0803 .

1-methyl-3-(2-methyl-1H-indol-3-yl)-1H-pyrrole-2,5-dione (4c) 


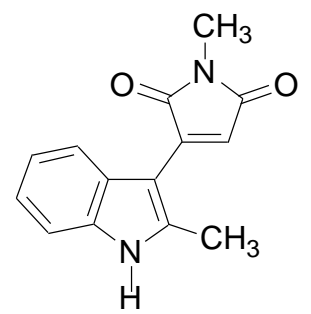

Yield 149 mg (83\%) as saffron yellow solid; Mp: 202-204 ${ }^{\circ} \mathrm{C}$. IR (KBr): 3436, 3317, 2923, 1764, 1692, 1604, 1437, 1383, 1223, 966, 841, $742 \mathrm{~cm}^{-1} .{ }^{\mathbf{1}}$ H NMR (400 MHz, DMSO-d $)$ ): $\delta 2.57$ (s, 3H), $2.97(\mathrm{~s}, 3 \mathrm{H}), 6.70(\mathrm{~s}, 1 \mathrm{H}), 7.11(\mathrm{dt}, J=14.7,7.1 \mathrm{~Hz}, 2 \mathrm{H}), 7.36(\mathrm{~d}, J=7.7 \mathrm{~Hz}, 1 \mathrm{H}), 7.74(\mathrm{~d}, J=7.5 \mathrm{~Hz}$, 1H), 11.80 (s, 1H); ${ }^{13}$ C NMR (101 MHz, DMSO-d d $_{6}: \delta 14.42,24.02,103.08,111.54,120.35,120.82$, $120.85,122.20,127.14,136.06,140.60,142.11,171.41,171.85 ; \mathbf{M S}(\mathbf{E I}): \mathrm{m} / \mathrm{z}=240[\mathrm{M}]^{+}, 225,211$, 183, 155, 154, 77. HRMS (EI): m/z calcd for $\mathrm{C}_{14} \mathrm{H}_{12} \mathrm{~N}_{2} \mathrm{O}_{2}: 240.0899$, found: 240.0903 .

1-phenyl-3-(2-methyl-1H-indol-3-yl)-1H-pyrrole-2,5-dione (4d)

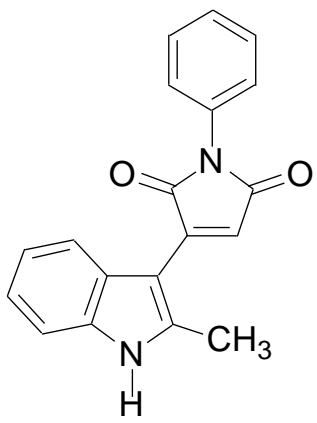

Yield $199 \mathrm{mg}$ (88\%) as brown solid; Mp: 140-142 ${ }^{\circ} \mathrm{C}$. IR (KBr): 3359, 2920, 2859, 1767, 1745, 1706, 1607, 1498, 1453, 1380, 1235, 960, 870, 745, $694 \mathrm{~cm}^{-1} .{ }^{1}$ H NMR (400 MHz, DMSO-d ) $_{\text {) }} \delta 2.60$ (s, 3H), $6.86(\mathrm{~s}, 1 \mathrm{H}), 7.12(\mathrm{dt}, J=14.7,7.1 \mathrm{~Hz}, 2 \mathrm{H}), 7.48-7.35(\mathrm{~m}, 4 \mathrm{H}), 7.51(\mathrm{t}, J=7.6 \mathrm{~Hz}, 2 \mathrm{H}), 7.80$ (d, $J=7.8 \mathrm{~Hz}, 1 \mathrm{H}), 11.87(\mathrm{~s}, 1 \mathrm{H}) ;{ }^{13} \mathrm{C}$ NMR (101 MHz, DMSO-d $\left.\boldsymbol{d}_{6}\right): \delta 14.52,103.06,111.60,120.52$, $120.75,120.95,122.31,127.16,127.38(2 \mathrm{C}), 127.92,129.26(2 \mathrm{C}), 132.65,136.12,141.11,142.03$, 170.16, 170.59; MS (EI): $\mathrm{m} / \mathrm{z}=302[\mathrm{M}]^{+}, 288,273,261,210,182,155,154,130,77$. HRMS (EI): $\mathrm{m} / \mathrm{z}$ calcd for $\mathrm{C}_{19} \mathrm{H}_{14} \mathrm{~N}_{2} \mathrm{O}_{2}: 302.1055$, found: 302.1061 .

1-benzyl-3-(2-methyl-1H-indol-3-yl)-1H-pyrrole-2,5-dione (4e) 


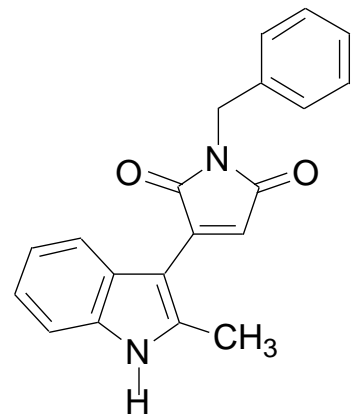

Yield $189 \mathrm{mg}$ (80\%) as saffron yellow solid; Mp: $174-176{ }^{\circ} \mathrm{C}$. IR (KBr): 3458, 3339, 2920, 1758, 1691, 1579, 1392, 1347, 1228, 954, 832, $732 \mathrm{~cm}^{-1} .{ }^{\mathbf{1}}$ H NMR (400 MHz, DMSO-d $\left.\boldsymbol{d}_{\boldsymbol{6}}\right): \delta 2.56$ (s, 3H), $4.69(\mathrm{~s}, 2 \mathrm{H}), 6.75(\mathrm{~s}, 1 \mathrm{H}), 7.10(\mathrm{dt}, J=14.7,7.1 \mathrm{~Hz}, 2 \mathrm{H}), 7.32(\mathrm{dt}, J=13.4,6.0 \mathrm{~Hz}, 6 \mathrm{H}), 7.75(\mathrm{~d}, J=$ $7.7 \mathrm{~Hz}, 1 \mathrm{H}), 11.84(\mathrm{~s}, 1 \mathrm{H}) ;{ }^{13} \mathbf{C}$ NMR (101 MHz, DMSO-d $\left.\boldsymbol{d}_{\mathbf{6}}\right): \delta 14.05,40.68,102.57,111.07,119.92$, $120.00,120.43,121.78,126.56,127.29(3 \mathrm{C}), 128.55(2 \mathrm{C}), 135.56,137.11,140.50,141.58,170.60$, 171.00; MS (EI): $\mathrm{m} / \mathrm{z}=316[\mathrm{M}]^{+}, 288,273,259,225,211,184,155,154,91,77$. HRMS (EI): $\mathrm{m} / \mathrm{z}$ calcd for $\mathrm{C}_{20} \mathrm{H}_{16} \mathrm{~N}_{2} \mathrm{O}_{2}: 316.1212$, found: 316.1209 .

\section{General procedure for the synthesis of $4 \mathrm{f}$ and $4 \mathrm{~g}$}

A mixture of indolylsuccinimide 6 (3.0 mmol, 1 equiv), $\mathrm{Pd}(\mathrm{OAc})_{2}(67.3 \mathrm{mg}, 0.3 \mathrm{mmol}, 0.1$ equiv), $\mathrm{Cu}(\mathrm{OAc})_{2}(817 \mathrm{mg}, 4.5 \mathrm{mmol}, 1.5$ equiv $)$ and $\mathrm{DMF} / \mathrm{DMSO}(10 \mathrm{~mL}, \mathrm{~V}: \mathrm{V}=1: 5)$ were stirred at $80{ }^{\circ} \mathrm{C}$ for $18 \mathrm{hr}$. The mixture was cooled to room temperature before quenched with $\mathrm{H}_{2} \mathrm{O}(10 \mathrm{~mL})$ and extracted with ethyl acetate $(100 \mathrm{~mL})$. Filtering out the insoluble substances, the organic phases were washed with $\mathrm{H}_{2} \mathrm{O}(3 \times 50 \mathrm{~mL})$, and dried $\left(\mathrm{Na}_{2} \mathrm{SO}_{4}\right)$. The solvent was removed and the residue was purified by silica gel column chromatography (eluent: acetone-PE, 1:8 and 1:12, respectively) to yield the pure product $\mathbf{4 f}$ and $\mathbf{4 g}$.

3-(1H-indol-3-yl)-1H-pyrrole-2,5-dione (4f)

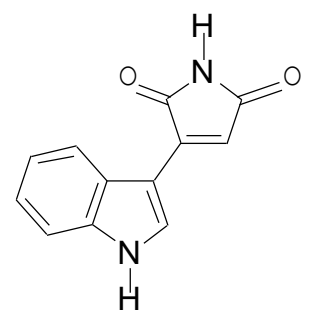

Yield $515 \mathrm{mg}$ (81\%) as saffron yellow solid; Mp: 224-226 ${ }^{\circ} \mathrm{C}$. IR (KBr): 3433, 3347, 3240, 3057, 1751, 1702, 1601, $740 \mathrm{~cm}^{-1} .{ }^{1}$ H NMR (400 MHz, DMSO-d $\left.\boldsymbol{d}_{6}\right) \delta 6.81(\mathrm{~s}, 1 \mathrm{H}), 7.24(\mathrm{dt}, J=22.2,7.3 \mathrm{~Hz}$, 
2H), $7.53(\mathrm{~d}, J=7.8 \mathrm{~Hz}, 1 \mathrm{H}), 7.98(\mathrm{~d}, J=7.8 \mathrm{~Hz}, 1 \mathrm{H}), 8.38(\mathrm{~s}, 1 \mathrm{H}), 10.78(\mathrm{~s}, 1 \mathrm{H}), 12.04(\mathrm{~s}, 1 \mathrm{H}) ;{ }^{13} \mathbf{C}$ NMR (101 MHz, DMSO-d $\left.\boldsymbol{d}_{\boldsymbol{6}}\right): \delta$ 105.80, 113.01, 115.73, 120.83, 121.85, 123.42, 126.05, 131.37, 137.12, 139.92, 173.62, 173.80; MS (EI): $\mathrm{m} / \mathrm{z}=212[\mathrm{M}]^{+}, 184,156,141,128,122,114$.

\section{1-phenyl-3-(1H-indol-3-yl)-1H-pyrrole-2,5-dione (4g)}

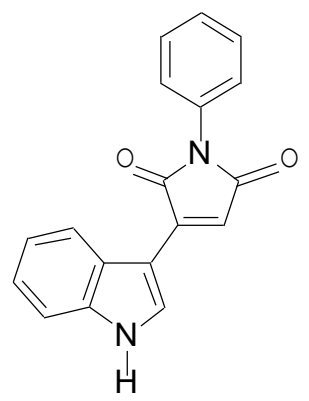

Yield $749 \mathrm{mg}(86 \%)$ as yellow brown solid; Mp: 216-218 ${ }^{\circ} \mathrm{C}$. IR (KBr): 3445, 3350, 1745, 1694, 1610, 1506, 806, $750 \mathrm{~cm}^{-1} .{ }^{1} \mathbf{H}$ NMR (400 MHz, DMSO-d $\left.\boldsymbol{d}_{6}\right): \delta 7.08(\mathrm{~s}, 1 \mathrm{H}), 7.27$ (dt, $J=15.0,7.0 \mathrm{~Hz}$, 2H), $7.46-7.36(\mathrm{~m}, 3 \mathrm{H}), 7.59-7.48(\mathrm{~m}, 3 \mathrm{H}), 8.07(\mathrm{~d}, J=7.7 \mathrm{~Hz}, 1 \mathrm{H}), 8.45(\mathrm{~d}, J=2.9 \mathrm{~Hz}, 1 \mathrm{H})$, 12.12 (s, 1H); ${ }^{13}$ C NMR (101 MHz, DMSO-d $\left.\boldsymbol{d}_{\mathbf{6}}\right): \delta 105.89,113.16,114.66,120.97,122.09,123.63$, $126.04,127.43(2 \mathrm{C}), 127.96,129.26(2 \mathrm{C}), 131.95,132.55,137.23,139.41,170.81,171.19 ;$ MS (EI): $\mathrm{m} / \mathrm{z}=288[\mathrm{M}]^{+}, 260,168,141,114,88$. HRMS (EI): m/z calcd for $\mathrm{C}_{18} \mathrm{H}_{12} \mathrm{~N}_{2} \mathrm{O}_{2}: 288.0899$, found: 288.0901.

\section{3,4-di-(2-methyl-1H-indol-3-yl)-1H-pyrrole-2,5-dione (5a)}

A mixture of 2-methylindole 1 (196.7 mg, $1.5 \mathrm{mmol}, 2$ equiv), maleimide $2 \mathbf{a}(0.75 \mathrm{mmol}, 1$ equiv), $\mathrm{Pd}(\mathrm{OAc})_{2}\left(16.8 \mathrm{mg}, 0.075 \mathrm{mmol}, 0.1\right.$ equiv), $\mathrm{Cu}(\mathrm{OAc})_{2}(204.3 \mathrm{mg}, 1.125 \mathrm{mmol}, 1.5$ equiv) and DMSO (3 mL) were stirred at $80{ }^{\circ} \mathrm{C}$ for $18 \mathrm{hr}$ (TLC monitoring, acetone-PE, 1:1 4). The mixture was cooled to room temperature before quenched with $\mathrm{H}_{2} \mathrm{O}(3 \mathrm{~mL})$ and extracted with ethyl acetate (50 mL). Filtering out the insolubes, the organic phases were washed with $\mathrm{H}_{2} \mathrm{O}(3 \times 25 \mathrm{~mL})$, and dried $\left(\mathrm{Na}_{2} \mathrm{SO}_{4}\right)$. The solvent was removed and the residue was purified by silica gel column chromatography (eluent: acetone-PE, 1:5 4) to yield the pure products $\mathbf{4 a}$ and $\mathbf{5 a}$. 


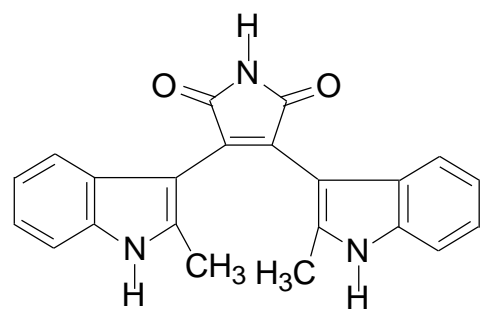

Yield $82 \mathrm{mg}$ (31\%) as red solid; Mp: 294-296 ${ }^{\circ} \mathrm{C}$. IR (KBr): 3379, 3048, 2917, 1754, 1705, 1617, 1545, 1458, 1341, 1232, 998, $742 \mathrm{~cm}^{-1} .{ }^{1}$ H NMR (400 MHz, DMSO-d $): \delta 1.97$ (s, 6H), 6.75 (t, $J=$ $7.5 \mathrm{~Hz}, 2 \mathrm{H}), 6.95(\mathrm{t}, J=7.5 \mathrm{~Hz}, 2 \mathrm{H}), 7.01(\mathrm{~d}, J=7.6 \mathrm{~Hz}, 2 \mathrm{H}), 7.22(\mathrm{~d}, J=8.0 \mathrm{~Hz}, 2 \mathrm{H}), 10.87(\mathrm{~s}, 1 \mathrm{H})$, 11.29 (s, 2H); ${ }^{13}$ C NMR (101 MHz, DMSO-d $)$ ): $\delta 13.47$ (2C), 103.75 (2C), 111.06 (2C), 119.57 (2C), 119.78 (2C), 121.20 (2C), 127.12 (2C), 132.35 (2C), 135.90 (2C), 137.65 (2C), 172.89 (2C); MS (EI): $\mathrm{m} / \mathrm{z}=355[\mathrm{M}]^{+}, 340,312,297,283,269,141$. HRMS (EI): $\mathrm{m} / \mathrm{z}$ calcd for $\mathrm{C}_{22} \mathrm{H}_{17} \mathrm{~N}_{3} \mathrm{O}_{2}: 355.1321$, found: 355.1319 .

\section{3,4-di-(1H-indol-3-yl)-1 $H$-pyrrole-2,5-dione (5b)}

A mixture of indole $1 \mathbf{a}(128.8 \mathrm{mg}, 1.1 \mathrm{mmol}, 1.1$ equiv), 3-(1H-indol-3-yl)-succinimide 6a $(224.2 \mathrm{mg}$, 1.0 mmol, 1 equiv), $\mathrm{Pd}(\mathrm{OAc})_{2}$ (22.4 mg, $0.1 \mathrm{mmol}, 0.1$ equiv), $\mathrm{Cu}(\mathrm{OAc})_{2}(272.4 \mathrm{mg}, 1.5 \mathrm{mmol}, 1.5$ equiv) and DMF/DMSO (3 mL, V:V = 1:5) were stirred at $80{ }^{\circ} \mathrm{C}$ for $18 \mathrm{hr}$. The mixture was cooled to room temperature before quenched with $\mathrm{H}_{2} \mathrm{O}(3 \mathrm{~mL})$ and extracted with ethyl acetate $(50 \mathrm{~mL})$. Filtering out the insoluble substances, the organic phases were washed with $\mathrm{H}_{2} \mathrm{O}(3 \times 25 \mathrm{~mL})$, and dried $\left(\mathrm{Na}_{2} \mathrm{SO}_{4}\right)$. The solvent was removed and the residue was purified by silica gel column chromatography (eluent: acetone-PE, 1:8 6) to yield the pure products $\mathbf{3 a}$ and $\mathbf{5 b}$.

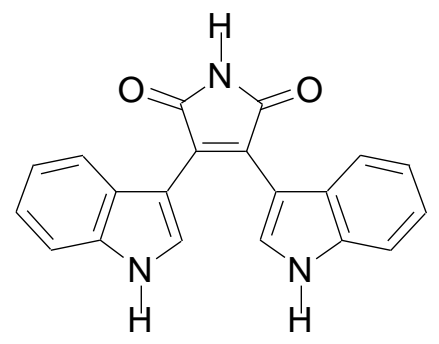

Yield $49 \mathrm{mg}$ (15\%) as red solid; Mp: 158-160 ${ }^{\circ} \mathrm{C}$. IR (KBr): 3388, 3055, 1754, 1693, 1620, 1530, 1428, 1341, 1232, 989, $744 \mathrm{~cm}^{-1} .{ }^{1}$ H NMR (400 MHz, DMSO-d $\left.\boldsymbol{d}_{\boldsymbol{\sigma}}\right): \delta 6.63(\mathrm{t}, J=7.5 \mathrm{~Hz}, 2 \mathrm{H}), 6.81(\mathrm{~d}$, $J=8.0 \mathrm{~Hz}, 2 \mathrm{H}), 6.98(\mathrm{t}, J=7.5 \mathrm{~Hz}, 2 \mathrm{H}), 7.37$ (d, $J=8.1 \mathrm{~Hz}, 2 \mathrm{H}), 7.74(\mathrm{~s}, 2 \mathrm{H}), 10.91(\mathrm{~s}, 1 \mathrm{H}), 11.67$ (s, 2H); ${ }^{13}$ C NMR (101 MHz, DMSO-d $\left.\boldsymbol{d}_{\boldsymbol{6}}\right): \delta 106.05$ (2C), 112.17 (2C), 119.75 (2C), 121.35 (2C), 122.02 
(2C), 125.88 (2C), 128.17 (2C), 129.54 (2C), 136.41 (2C), 173.41 (2C); MS (EI): m/z = $327[\mathrm{M}]^{+}$, 283, 256, 255, 207, 128, 88. HRMS (EI): m/z calcd for $\mathrm{C}_{20} \mathrm{H}_{13} \mathrm{~N}_{3} \mathrm{O}_{2}: 327.1008$, found: 327.1005.

\section{3-(1H-indol-3-yl)-4-(5-methoxy- $1 H$-indol-3-yl)-1H-pyrrole-2,5-dione (5c)}

A mixture of 5-methoxyindole $\mathbf{1 b}(161.9 \mathrm{mg}, 1.1 \mathrm{mmol}, 1.1$ equiv), 3-(1H-indol-3-yl)-succinimide $\mathbf{6 a}$ (224.2 mg, $1.0 \mathrm{mmol}, 1$ equiv), $\mathrm{Pd}(\mathrm{OAc})_{2}\left(22.4 \mathrm{mg}, 0.1 \mathrm{mmol}, 0.1\right.$ equiv), $\mathrm{Cu}(\mathrm{OAc})_{2}(272.4 \mathrm{mg}, 1.5$ mmol, 1.5 equiv) and DMF/DMSO ( $3 \mathrm{~mL}, \mathrm{~V}: \mathrm{V}=1: 5)$ were stirred at $80{ }^{\circ} \mathrm{C}$ for $18 \mathrm{hr}$. The mixture was cooled to room temperature before quenched with $\mathrm{H}_{2} \mathrm{O}(3 \mathrm{~mL})$ and extracted with ethyl acetate (50 mL). Filtering out the insoluble substances, the organic phases were washed with $\mathrm{H}_{2} \mathrm{O}(3 \times 25$ $\mathrm{mL})$, and dried $\left(\mathrm{Na}_{2} \mathrm{SO}_{4}\right)$. The solvent was removed and the residue was purified by silica gel column chromatography (eluent: acetone-PE, 1:5 4) to yield the pure products $\mathbf{3 I}$ and $\mathbf{5 c}$.

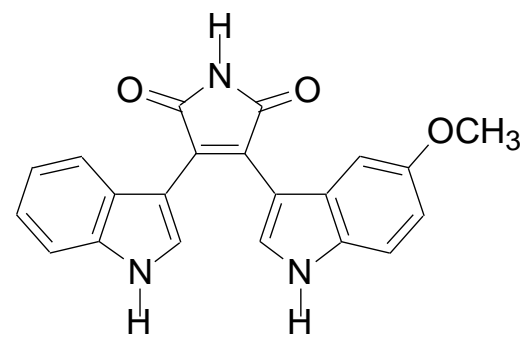

Yield $75 \mathrm{mg}$ (21\%) as red solid; Mp: 288-290 ${ }^{\circ} \mathrm{C}$. IR (KBr): 3401, 3349, 3051, 2923, 1703, 1620, 1569, 1524, 1476, 1339, 797, $747 \mathrm{~cm}^{-1} .{ }^{1}$ H NMR (400 MHz, DMSO-d d $_{\text {) }} \delta 2.97$ (s, 3H), 6.07 (d, $J=$ $2.2 \mathrm{~Hz}, 1 \mathrm{H}), 6.54(\mathrm{dd}, J=8.7,2.4 \mathrm{~Hz}, 1 \mathrm{H}), 6.69(\mathrm{t}, J=7.5 \mathrm{~Hz}, 1 \mathrm{H}), 6.95(\mathrm{~d}, J=8.0 \mathrm{~Hz}, 1 \mathrm{H}), 7.00(\mathrm{t}, J$ $=7.6 \mathrm{~Hz}, 1 \mathrm{H}), 7.22(\mathrm{~d}, J=8.7 \mathrm{~Hz}, 1 \mathrm{H}), 7.38(\mathrm{~d}, J=8.1 \mathrm{~Hz}, 1 \mathrm{H}), 7.65(\mathrm{~d}, J=2.6 \mathrm{~Hz}, 1 \mathrm{H}), 7.85(\mathrm{~d}, J=$ $2.8 \mathrm{~Hz}, 1 \mathrm{H}), 10.89(\mathrm{~s}, 1 \mathrm{H}), 11.60(\mathrm{~s}, 1 \mathrm{H}), 11.64(\mathrm{~s}, 1 \mathrm{H}) ;{ }^{13}$ C NMR (101 MHz, DMSO-d $\left.\boldsymbol{d}_{6}\right): \delta$ 54.40, $103.00,105.87,106.46,112.01,112.52,112.74,119.77,121.13,122.11,126.12,126.53,126.73$, $128.96,129.28,130.20,131.26,136.32,153.50,173.49,173.53 ; \mathbf{M S}(\mathbf{E I}): \mathrm{m} / \mathrm{z}=357[\mathrm{M}]^{+}, 340,325$, 314, 286, 271, 242, 227, 212, 199, 156, 141, 122. HRMS (EI): m/z calcd for $\mathrm{C}_{21} \mathrm{H}_{15} \mathrm{~N}_{3} \mathrm{O}_{3}: 357.1113$, found: 357.1120 .

\section{1-phenyl-3,4-di-(1H-indol-3-yl)-1H-pyrrole-2,5-dione (5d)}

A mixture of indole $\mathbf{1 a}(128.8 \mathrm{mg}, 1.1 \mathrm{mmol}, 1.1$ equiv), $N$-phenyl-3-(1H-indol-3-yl) -maleimide $\mathbf{4 g}$ (288.3 mg, $1.0 \mathrm{mmol}, 1$ equiv), $\mathrm{Pd}(\mathrm{OAc})_{2}\left(22.4 \mathrm{mg}, 0.1 \mathrm{mmol}, 0.1\right.$ equiv), $\mathrm{Cu}(\mathrm{OAc})_{2}(272.4 \mathrm{mg}, 1.5$ mmol, 1.5 equiv) and DMSO $(3 \mathrm{~mL})$ were stirred at $80{ }^{\circ} \mathrm{C}$ for $18 \mathrm{hr}$. The mixture was cooled to room 
temperature before quenched with $\mathrm{H}_{2} \mathrm{O}(3 \mathrm{~mL})$ and extracted with ethyl acetate $(50 \mathrm{~mL})$. Filtering out the insoluble substance, the organic phases were washed with $\mathrm{H}_{2} \mathrm{O}(3 \times 25 \mathrm{~mL})$, and dried $\left(\mathrm{Na}_{2} \mathrm{SO}_{4}\right)$. The solvent was removed and the residue was purified by silica gel column chromatography (eluent: acetone-PE, 1:8 6) to yield the pure products $\mathbf{3 g}$ and $\mathbf{5 d}$.

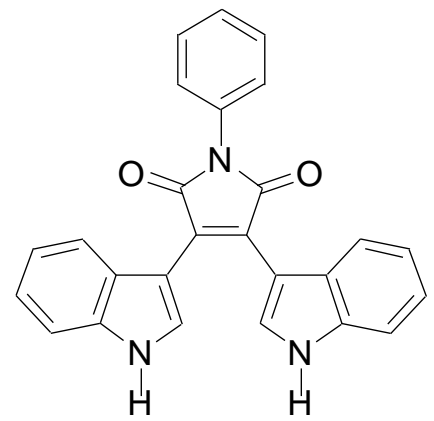

Yield 149 mg (37\%) as red solid; Mp: 210-212 ${ }^{\circ} \mathrm{C}$. IR (KBr): 3385, 3048, 1748, 1697, 1623, 1530, 1389, 1235, $742 \mathrm{~cm}^{-1} .{ }^{1}$ H NMR (400 MHz, DMSO-d $\left.\boldsymbol{d}_{6}\right): \delta 6.68(\mathrm{t}, J=7.5 \mathrm{~Hz}, 2 \mathrm{H}), 6.90(\mathrm{~d}, J=8.1 \mathrm{~Hz}$, 2H), $7.01(\mathrm{t}, J=7.6 \mathrm{~Hz}, 2 \mathrm{H}), 7.42(\mathrm{t}, J=8.3 \mathrm{~Hz}, 3 \mathrm{H}), 7.54(\mathrm{q}, J=8.3 \mathrm{~Hz}, 4 \mathrm{H}), 7.83(\mathrm{~d}, J=2.6 \mathrm{~Hz}$, 2H), 11.75 (s, 2H); ${ }^{13}$ C NMR (101 MHz, DMSO-d d $_{\text {) }} \delta 106.03$ (2C), 112.31 (2C), 119.91 (2C), 121.53 (2C), 122.20 (2C), 125.87 (2C), 127.44 (2C), 127.55 (2C), 127.89, 129.29 (2C), 129.99 (2C), 133.01, 136.51 (2C), 170.97 (2C); MS (EI): $\mathrm{m} / \mathrm{z}=403[\mathrm{M}]^{+}, 311,283,256,255,128$. HRMS (EI): $\mathrm{m} / \mathrm{z}$ calcd for $\mathrm{C}_{26} \mathrm{H}_{17} \mathrm{~N}_{3} \mathrm{O}_{2}: 403.1321$, found: 403.1316 .

\section{1-phenyl-3-(1H-indol-3-yl)-4-(5-methoxy- $1 H$-indol-3-yl)-1H-pyrrole-2,5-dione (5e)}

A mixture of 5-methoxyindole $\mathbf{1 b}(161.9 \mathrm{mg}, 1.1 \mathrm{mmol}, 1.1$ equiv), $N$-phenyl-3-( $1 H$-indol-3-yl) -maleimide 4g (288.3 mg, 1.0 mmol, 1 equiv), $\mathrm{Pd}(\mathrm{OAc})_{2}$ (22.4 mg, $0.1 \mathrm{mmol}, 0.1$ equiv), $\mathrm{Cu}(\mathrm{OAc})_{2}$ (272.4 mg, $1.5 \mathrm{mmol}, 1.5$ equiv) and DMF/DMSO (3 mL, V:V = 1:5) were stirred at $80{ }^{\circ} \mathrm{C}$ for $18 \mathrm{hr}$. The mixture was cooled to room temperature before quenched with $\mathrm{H}_{2} \mathrm{O}(3 \mathrm{~mL})$ and extracted with ethyl acetate $(50 \mathrm{~mL})$. Filtering out the insoluble substances, the organic phases were washed with $\mathrm{H}_{2} \mathrm{O}(3 \times 25 \mathrm{~mL})$, and dried $\left(\mathrm{Na}_{2} \mathrm{SO}_{4}\right)$. The solvent was removed and the residue was purified by silica gel column chromatography (eluent: acetone-PE, 1:6 5) to yield the pure products $\mathbf{3 m}$ and $\mathbf{5 e}$ 


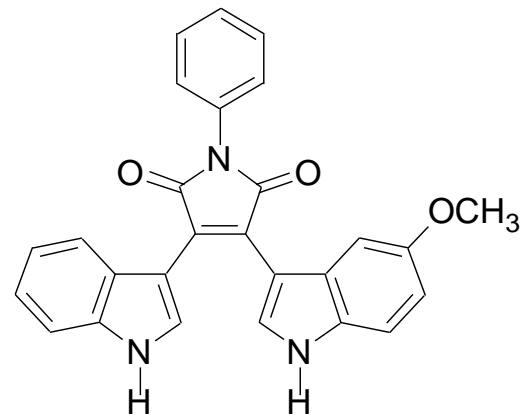

Yield $182 \mathrm{mg}$ (42\%) as red solid; Mp: 240-242 ${ }^{\circ} \mathrm{C}$. IR (KBr): 3383, 2920, 1745, 1696, 1628, 1527 , 1485, 1383, 1111, 886, $744 \mathrm{~cm}^{-1}{ }^{1}{ }^{1}$ H NMR (400 MHz, DMSO-d d $_{\text {) }} \delta 3.00$ (s, 3H), $6.14(\mathrm{~s}, 1 \mathrm{H}), 6.56$ (d, $J=8.6 \mathrm{~Hz}, 1 \mathrm{H}), 6.72(\mathrm{t}, J=7.5 \mathrm{~Hz}, 1 \mathrm{H}), 7.03(\mathrm{t}, J=8.0 \mathrm{~Hz}, 2 \mathrm{H}), 7.25(\mathrm{~d}, J=8.7 \mathrm{~Hz}, 1 \mathrm{H}), 7.42(\mathrm{t}, J=$ $7.1 \mathrm{~Hz}, 2 \mathrm{H}), 7.52(\mathrm{q}, J=7.7 \mathrm{~Hz}, 4 \mathrm{H}), 7.72(\mathrm{~s}, 1 \mathrm{H}), 7.92(\mathrm{~d}, J=2.2 \mathrm{~Hz}, 1 \mathrm{H}), 11.66(\mathrm{~s}, 1 \mathrm{H}), 11.69(\mathrm{~s}, 1 \mathrm{H})$; ${ }^{13}$ C NMR (101 MHz, DMSO-d $\left.\boldsymbol{d}_{\boldsymbol{6}}\right): \delta 54.45,103.14,105.85,106.41,112.13,112.69,112.88,119.91$, 121.30, 122.26, 126.10, 126.11, 126.50, 127.44 (2C), 127.88, 128.66, 129.28 (2C), 129.37, 130.60, 131.34, 133.01, 136.41, 153.63, 170.94, 170.99; MS (EI): m/z $=433[\mathrm{M}]^{+}, 416,401,341,313,286$, 271, 243, 122. HRMS (EI): m/z calcd for $\mathrm{C}_{27} \mathrm{H}_{19} \mathrm{~N}_{3} \mathrm{O}_{3}: 433.1426$, found: 433.1425 .

\section{General procedure for the synthesis of $7 a$ and $7 b$}

A mixture of indolylmaleimide 4 ( $0.75 \mathrm{mmol}, 1$ equiv), maleimide 2 ( $0.75 \mathrm{mmol}, 1$ equiv), $\mathrm{Pd}(\mathrm{OAc})_{2}$ (16.8 mg, $0.075 \mathrm{mmol}, 0.1$ equiv), $\mathrm{Cu}(\mathrm{OAc})_{2}(204.3 \mathrm{mg}, 1.125 \mathrm{mmol}, 1.5$ equiv) and DMF/DMSO (3 $\mathrm{mL}, \mathrm{V}: \mathrm{V}=1: 5$ ) were stirred at $80{ }^{\circ} \mathrm{C}$ for $18 \mathrm{hr}$ (TLC monitoring, acetone-PE, 1:1 4). The mixture was cooled to room temperature before quenched with $\mathrm{H}_{2} \mathrm{O}(3 \mathrm{~mL})$ and extracted with ethyl acetate $(250$ $\mathrm{mL})$. Filtering out the insoluble substances, the organic phases were washed with $\mathrm{H}_{2} \mathrm{O}(3 \times 25 \mathrm{~mL})$, and dried $\left(\mathrm{Na}_{2} \mathrm{SO}_{4}\right)$. The dried mixture was concentrated and filtered to yield the pure products. The products were characterized by IR, ${ }^{1} \mathrm{H}$ NMR, ${ }^{13} \mathrm{C}$ NMR, MS and HRMS analyses.

\section{$1 H$-dipyrrolo[3,4-a:3',4'-c] carbazole-1,3,4,6(2H,5H,7H)-tetrone (7a)}

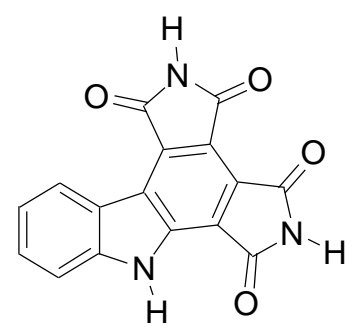

Yield $155 \mathrm{mg}$ (68\%) as brown solid; Mp: >350 ${ }^{\circ} \mathrm{C}$. IR (KBr): 3438, 1725, 1636, 1434, 1287, 883, 
$726 \mathrm{~cm}^{-1} .{ }^{1} \mathrm{H}$ NMR (400 MHz, DMSO-d $\left.\boldsymbol{d}_{6}\right): \delta 7.43(\mathrm{~s}, 1 \mathrm{H}), 7.69$ (s, 1H), 7.77 (s, 1H), $9.00(\mathrm{~s}, 1 \mathrm{H})$, 11.60 (s, 2H), 12.77 (s, 1H); ${ }^{13}$ C NMR (101 MHz, DMSO-d 6 ): $\delta$ 112.88, 117.95, 119.37, 119.49, $121.54,124.28,125.52,125.70,129.94,131.49,136.79,144.10,168.48(2 \mathrm{C}), 168.62,169.24 ;$ MS (EI): $\mathrm{m} / \mathrm{z}=305[\mathrm{M}]^{+}, 261,234,190,163$. HRMS (EI): $\mathrm{m} / \mathrm{z}$ calcd for $\mathrm{C}_{16} \mathrm{H}_{7} \mathrm{~N}_{3} \mathrm{O}_{4}:$ 305.0437, found: 305.0441.

2,5-diphenyl-1H-dipyrrolo[3,4-a:3',4'-c] carbazole-1,3,4,6(2H,5H,7H)-tetrone (7b)

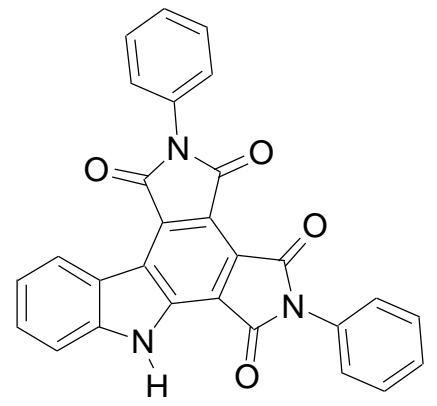

Yield $288 \mathrm{mg}(84 \%)$ as yellow solid; $\mathbf{M p : ~ > 3 5 0 ~}{ }^{\circ} \mathrm{C}$. IR (KBr): 3622, 3519, 3096, 3050, 1770, 1715, 1607, 1495, 1460, 1369, 1187, 1104, $747 \mathrm{~cm}^{-1} .{ }^{1}$ H NMR (400 MHz, DMSO-d $\left.)_{6}\right): \delta .45(\mathrm{t}, J=7.6 \mathrm{~Hz}$, 1H), $7.51(\mathrm{t}, J=6.5 \mathrm{~Hz}, 2 \mathrm{H}), 7.56-7.63(\mathrm{~m}, 8 \mathrm{H}), 7.71(\mathrm{t}, J=7.5 \mathrm{~Hz}, 1 \mathrm{H}), 7.81(\mathrm{~d}, J=8.0 \mathrm{~Hz}, 1 \mathrm{H})$, $9.01(\mathrm{~d}, J=8.0 \mathrm{~Hz}, 1 \mathrm{H}), 12.93(\mathrm{~s}, 1 \mathrm{H}) ;{ }^{13} \mathbf{C}$ NMR (101 MHz, DMSO-d $\left.)\right): \delta 113.01,113.27,116.84$, $118.23,119.29,121.83,124.46,124.64,125.54,127.43$ (2C), 127.54 (2C), 128.12, 128.79 (2C), 128.87 (2C), 130.34, 131.71, 131.85, 134.72, 136.99, 144.30, 163.94 (2C), 164.23, 166.76; MS (EI): $\mathrm{m} / \mathrm{z}=457[\mathrm{M}]^{+}, 413,384,368,336,253,189,163$. HRMS (EI): $\mathrm{m} / \mathrm{z}$ calcd for $\mathrm{C}_{28} \mathrm{H}_{15} \mathrm{~N}_{3} \mathrm{O}_{4}: 457.1063$, found: 457.1068 .

General procedure for the synthesis of compounds $8 a-b^{1}$ and $9 a-9 b$

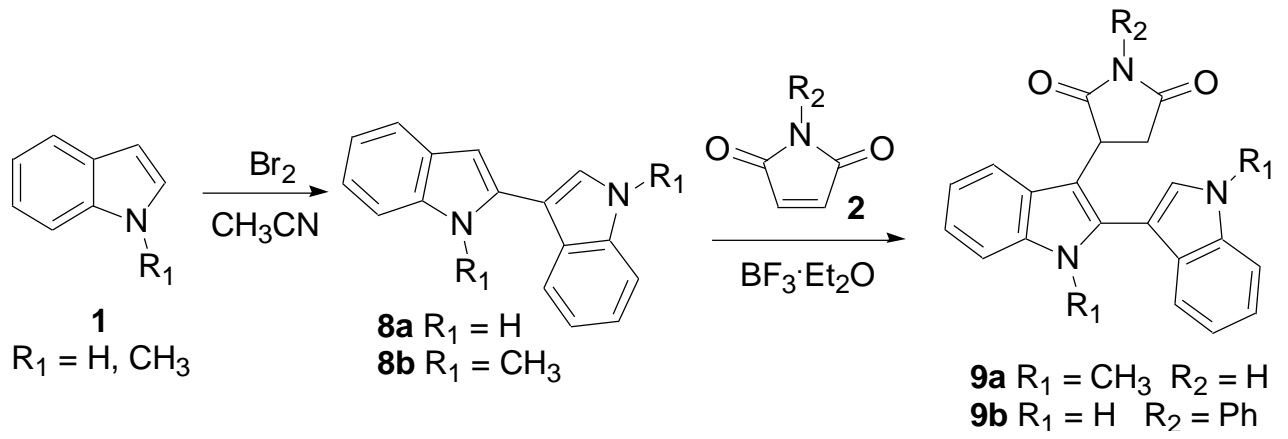

Indole 1 (20 mmol, 1 equiv) was stirred in acetonitrile $(30 \mathrm{~mL})$ and bromine (10 mmol, 0.5 equiv) in acetonitrile $(5 \mathrm{~mL})$ was added over $20 \mathrm{~min}$. The mixture was stirred overnight. And pale brown solid 
was filtered and washed with saturated sodium bicarbonate and recrystallized with EtOH to yield the pure products 8 .

A mixture of 2,3'-biindole 8 ( 3 mmol, 1 equiv), maleimide 2 ( $3.3 \mathrm{mmol}, 1.1$ equiv), $\mathrm{BF}_{3} \mathrm{Et}_{2} \mathrm{O}$ ( $0.085 \mathrm{~g}$, $0.6 \mathrm{mmol}, 0.2$ equiv) and 1,2-dichloroethane $(20 \mathrm{~mL})$ were stirred at $85{ }^{\circ} \mathrm{C}$ for 12 hours. The mixture was cooled to room temperature. The solvent was removed. The residue was washed with $\mathrm{H}_{2} \mathrm{O}$, extracted with ethyl acetate and then purified by silica gel column chromatography (eluent: ethyl acetate-PE, 1:4 3) to yield the pure product 9.

(1) Robertson, N.; Parsons, S.; MacLean, E.J.; Coxall, R.A.; Mount, A.R. J. Mater. Chem., 2000, 10, 2043.

\section{2,3'-bi-1H-indole (8a)}

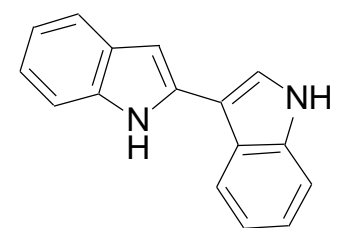

Yield $1.62 \mathrm{~g} \mathrm{(70 \% )}$ as brown solid; Mp: 198-200 ${ }^{\circ} \mathrm{C}$. IR (KBr): 3401, 1623, 1456, 1308, 1239, 1111 , 776, $743 \mathrm{~cm}^{-1} .{ }^{1} \mathbf{H}$ NMR (400 MHz, DMSO- $\left.d_{6}\right): \delta 6.77(\mathrm{~s}, 1 \mathrm{H}), 6.97(\mathrm{t}, J=7.4 \mathrm{~Hz}, 1 \mathrm{H}), 7.04(\mathrm{t}, J=$ $7.4 \mathrm{~Hz}, 1 \mathrm{H}), 7.24-7.13(\mathrm{~m}, 2 \mathrm{H}), 7.36(\mathrm{~d}, J=7.9 \mathrm{~Hz}, 1 \mathrm{H}), 7.49$ (dd, $J=11.4,7.9 \mathrm{~Hz}, 2 \mathrm{H}), 7.87$ (d, $J=$ $2.0 \mathrm{~Hz}, 1 \mathrm{H}), 8.01(\mathrm{~d}, J=7.6 \mathrm{~Hz}, 1 \mathrm{H}), 11.21(\mathrm{~s}, 1 \mathrm{H}), 11.41(\mathrm{~s}, 1 \mathrm{H}) .{ }^{13} \mathbf{C}$ NMR $\left(101 \mathrm{MHz}, \mathrm{DMSO}-d_{6}\right)$ : $\delta 97.28,108.93,110.90,112.43,119.30,119.55,120.11,120.25,120.79,122.22,123.64,125.13$, $129.70,134.62,136.47,137.14$

\section{1,1'-dimethyl-2,3'-bi-1H-indole (8b)}

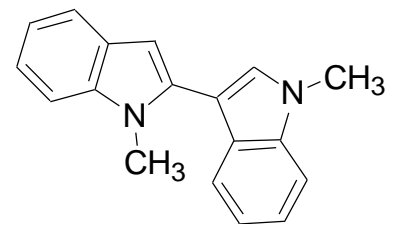

Yield $1.69 \mathrm{~g}(65 \%)$ as white solid; Mp: $120-122{ }^{\circ} \mathrm{C}$. IR (KBr): 3048, 2936, 2369, 1578, 1459, 1386, 1320, 1246, 1143, 1011, 947, 779, 743, $665 \mathrm{~cm}^{-1} .{ }^{\mathbf{1}} \mathbf{H}$ NMR (400 MHz, $\left.\mathrm{CDCl}_{3}\right): \delta 3.76(\mathrm{~s}, 3 \mathrm{H}), 3.88(\mathrm{~s}$, 3H), $6.56(\mathrm{~s}, 1 \mathrm{H}), 7.14(\mathrm{t}, J=7.4 \mathrm{~Hz}, 1 \mathrm{H}), 7.22(\mathrm{dt}, J=14.5,7.3 \mathrm{~Hz}, 3 \mathrm{H}), 7.31(\mathrm{t}, J=7.6 \mathrm{~Hz}, 1 \mathrm{H})$, $7.38(\mathrm{dd}, J=11.6,8.3 \mathrm{~Hz}, 2 \mathrm{H}), 7.64(\mathrm{~d}, J=7.8 \mathrm{~Hz}, 1 \mathrm{H}), 7.70(\mathrm{~d}, J=7.9 \mathrm{~Hz}, 1 \mathrm{H}) .{ }^{13} \mathbf{C}$ NMR $(101$ 
$\left.\mathrm{MHz}, \mathrm{CDCl}_{3}\right): \delta 31.10,33.07,100.77,107.31,109.40,109.57,119.67,120.09,120.29,120.37,121.10$, $122.38,127.69,128.40,128.67,135.53,136.97,138.07$

\section{3-(1,1'-dimethyl-2,3'-bi-1H-indolyl-3-yl)-pyrrolidine-2,5-dione (9a)}

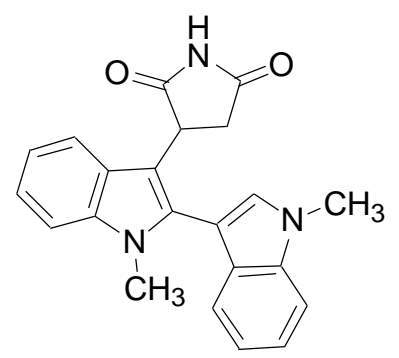

Yield $0.59 \mathrm{~g} \mathrm{(55 \% )}$ as white solid; Mp: $148-150{ }^{\circ} \mathrm{C}$. IR (KBr): 3477, 3234, 3048, 2936, 1777, 1709, 1620, 1517, 1469, 1351, 1251, 1175, 1021, 915, 825, 741, $669 \mathrm{~cm}^{-1} .{ }^{1} \mathbf{H}$ NMR (400 MHz, DMSO- $\left.d_{6}\right)$ : $\delta 2.58(\mathrm{dd}, J=18.0,5.3 \mathrm{~Hz}, 0.5 \mathrm{H}), 2.86(\mathrm{dd}, J=18.2,4.2 \mathrm{~Hz}, 0.5 \mathrm{H}), 3.08(\mathrm{dt}, J=20.7,10.4 \mathrm{~Hz}, 1 \mathrm{H})$, $3.61(\mathrm{~s}, 3 \mathrm{H}), 3.92(\mathrm{~s}, 3 \mathrm{H}), 4.16(\mathrm{dd}, J=16.4,9.5 \mathrm{~Hz}, 1 \mathrm{H}), 7.10(\mathrm{dd}, J=19.2,11.8 \mathrm{~Hz}, 2 \mathrm{H}), 7.25(\mathrm{dt}, J$ $=17.6,7.6 \mathrm{~Hz}, 3 \mathrm{H}), 7.38(\mathrm{~d}, J=7.7 \mathrm{~Hz}, 0.5 \mathrm{H}), 7.53(\mathrm{~d}, J=8.1 \mathrm{~Hz}, 1 \mathrm{H}), 7.61(\mathrm{dd}, J=16.9,8.6 \mathrm{~Hz}$, 2.5H), $11.41(\mathrm{~s}, 1 \mathrm{H}) .{ }^{13} \mathbf{C}$ NMR (101 MHz, DMSO- $\left.d_{6}\right): \delta 31.17,31.30,33.24,37.41,38.37,103.63$, $103.77,109.28,109.70,110.76,110.89,117.88,119.81,120.08,120.60,121.74,122.31,125.69$, $127.79,127.88,131.49,133.93,137.13,137.20,137.62,178.35,178.65,180.81,181.17$; MS (ESI): $\mathrm{m} / \mathrm{z}=358.2[\mathrm{M}+\mathrm{H}] . \mathbf{H R M S}(\mathbf{E S I}): \mathrm{m} / \mathrm{z}$ calcd for $\mathrm{C}_{22} \mathrm{H}_{20} \mathrm{~N}_{3} \mathrm{O}_{2}: 358.1550$, found 358.1550 .

\section{1-phenyl-3-(2,3'-bi-1H-indolyl-3-yl)-pyrrolidine-2,5-dione (9b)}

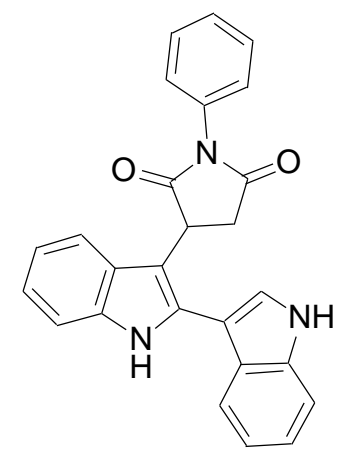

Yield $1.09 \mathrm{~g}(90 \%)$ as white solid; Mp: $180-182^{\circ} \mathrm{C}$. IR (KBr):3391, 3051, 2923, 1770, 1705, 1633, 1591, 1495, 1450, 1381, 1352, 1239, 1183, 1018, 925, 825, 743,697 $\mathrm{cm}^{-1} .{ }^{1} \mathbf{H}$ NMR (400 MHz, DMSO- $\left.d_{6}\right): \delta 3.01(\mathrm{dd}, J=18.2,5.3 \mathrm{~Hz}, 1 \mathrm{H}), 3.46-3.36(\mathrm{~m}, 1 \mathrm{H}), 4.62(\mathrm{dd}, J=9.7,5.3 \mathrm{~Hz}, 1 \mathrm{H}), 7.04$ (t, $J=7.4 \mathrm{~Hz}, 1 \mathrm{H}), 7.13(\mathrm{dd}, J=13.8,7.2 \mathrm{~Hz}, 2 \mathrm{H}), 7.21(\mathrm{t}, J=7.5 \mathrm{~Hz}, 1 \mathrm{H}), 7.32(\mathrm{~d}, J=7.7 \mathrm{~Hz}, 3 \mathrm{H})$, 
$7.44(\mathrm{t}, J=8.3 \mathrm{~Hz}, 2 \mathrm{H}), 7.53(\mathrm{t}, J=7.7 \mathrm{~Hz}, 3 \mathrm{H}), 7.66(\mathrm{~d}, J=2.0 \mathrm{~Hz}, 1 \mathrm{H}), 7.77(\mathrm{~d}, J=7.9 \mathrm{~Hz}, 1 \mathrm{H})$, $11.31(\mathrm{~s}, 1 \mathrm{H}), 11.59(\mathrm{~s}, 1 \mathrm{H}) .{ }^{13} \mathrm{C}$ NMR (101 MHz, DMSO- $\left.d_{6}\right): \delta 36.83,38.55,107.02,107.20,112.04$, $112.37,117.66,119.67,120.04,120.17,121.38,122.35,125.80,126.56,126.68,127.55,128.82$, 129.45, 132.94, 133.25, 136.75, 136.79, 176.30, 178.79; MS (ESI): m/z = 406.2 [M+H]. HRMS (ESI): $\mathrm{m} / \mathrm{z}$ calcd for $\mathrm{C}_{26} \mathrm{H}_{20} \mathrm{~N}_{3} \mathrm{O}_{2}: 406.1550$, found 406.1550 .

General procedure for the synthesis of compound 3 from 9 in the presence of $\mathrm{SeO}_{2}$

A mixture of compound 9 (1.5 mmol, 1 equiv), $\mathrm{SeO}_{2}(0.28 \mathrm{~g}, 2.55 \mathrm{mmol}, 1.5$ equiv) and 1,4-dioxane $(10 \mathrm{~mL})$ were stirred at $110^{\circ} \mathrm{C}$ for $12 \mathrm{hr}$. The solvent was removed and the mixture was extracted with $\mathrm{H}_{2} \mathrm{O}(3 \times 50 \mathrm{~mL})$ and ethyl acetate $(100 \mathrm{~mL})$. Filtering out the insoluble substances, and dried $\left(\mathrm{Na}_{2} \mathrm{SO}_{4}\right)$. The solvent was removed and the residue was purified by silica gel column chromatography to yield the pure indolopyrrolocarbazoles $\mathbf{3}$ (3c for $53 \%$ yield and $\mathbf{3 g}$ for $42 \%$ yield).

General procedure for the synthesis of products 3 from 9 in the palladium-catalyzed protocol A mixture of compounds 9 (0.15 mmol, 1 equiv), $\mathrm{Pd}(\mathrm{OAc})_{2}(3.4 \mathrm{mg}, 0.015 \mathrm{mmol}, 0.1$ equiv), $\mathrm{Cu}(\mathrm{OAc})_{2}\left(40.8 \mathrm{mg}, 0.225 \mathrm{mmol}, 1.5\right.$ equiv) and DMF/DMSO $(3 \mathrm{~mL}, \mathrm{~V}: \mathrm{V}=1: 5)$ were stirred at $80{ }^{\circ} \mathrm{C}$ for $18 \mathrm{hr}$. The mixture was cooled to room temperature before quenched with $\mathrm{H}_{2} \mathrm{O}(3 \mathrm{~mL})$ and extracted with ethyl acetate $(30 \mathrm{~mL})$. Filtering out the insoluble substances, the organic phases were washed with $\mathrm{H}_{2} \mathrm{O}(3 \times 10 \mathrm{~mL})$, and dried $\left(\mathrm{Na}_{2} \mathrm{SO}_{4}\right)$. The solvent was removed and the residue was purified by silica gel column chromatography to yield the pure indolopyrrolocarbazoles $\mathbf{3}$ (3c for $72 \%$ yield and $\mathbf{3 g}$ for $78 \%$ yield). 


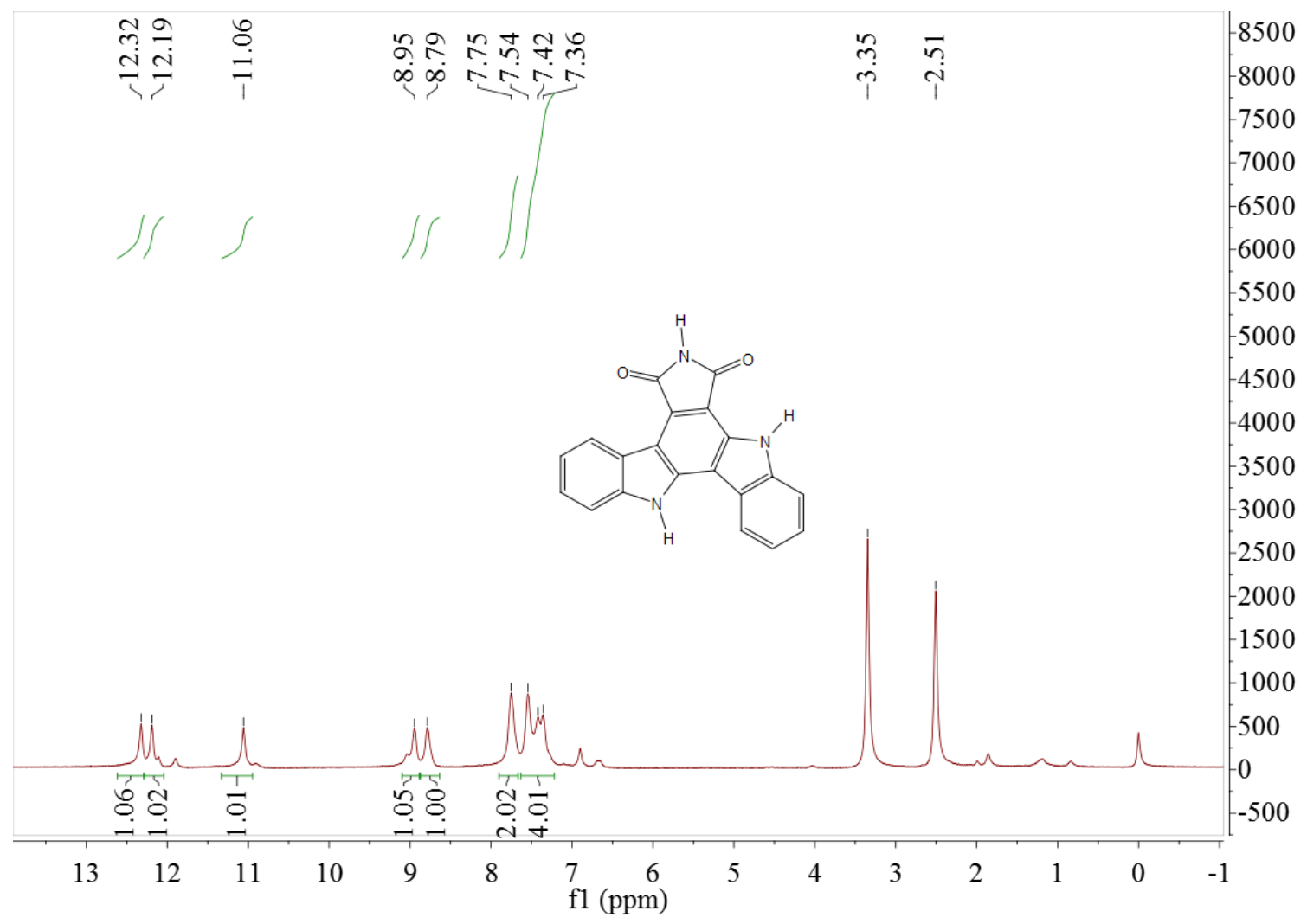

${ }^{1} \mathrm{H}$ NMR spectrum of $5 H$-indolo[3,2-a]pyrrolo[3,4-c]carbazole-6,8(7H,13H)-dione (3a)

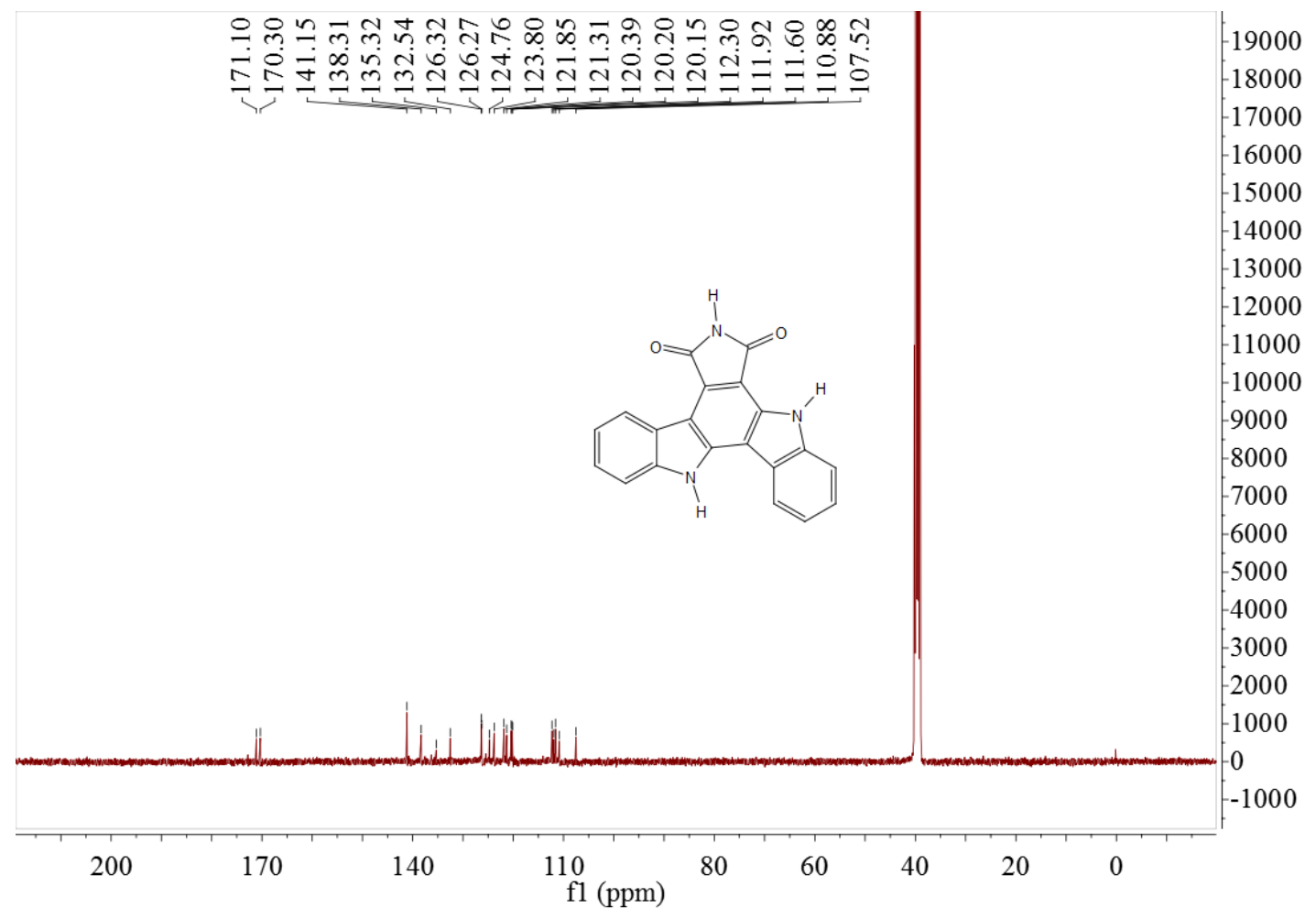

${ }^{13} \mathrm{C}$ NMR spectrum of $5 H$-indolo[3,2-a]pyrrolo[3,4-c]carbazole-6,8(7H,13H)-dione (3a) 


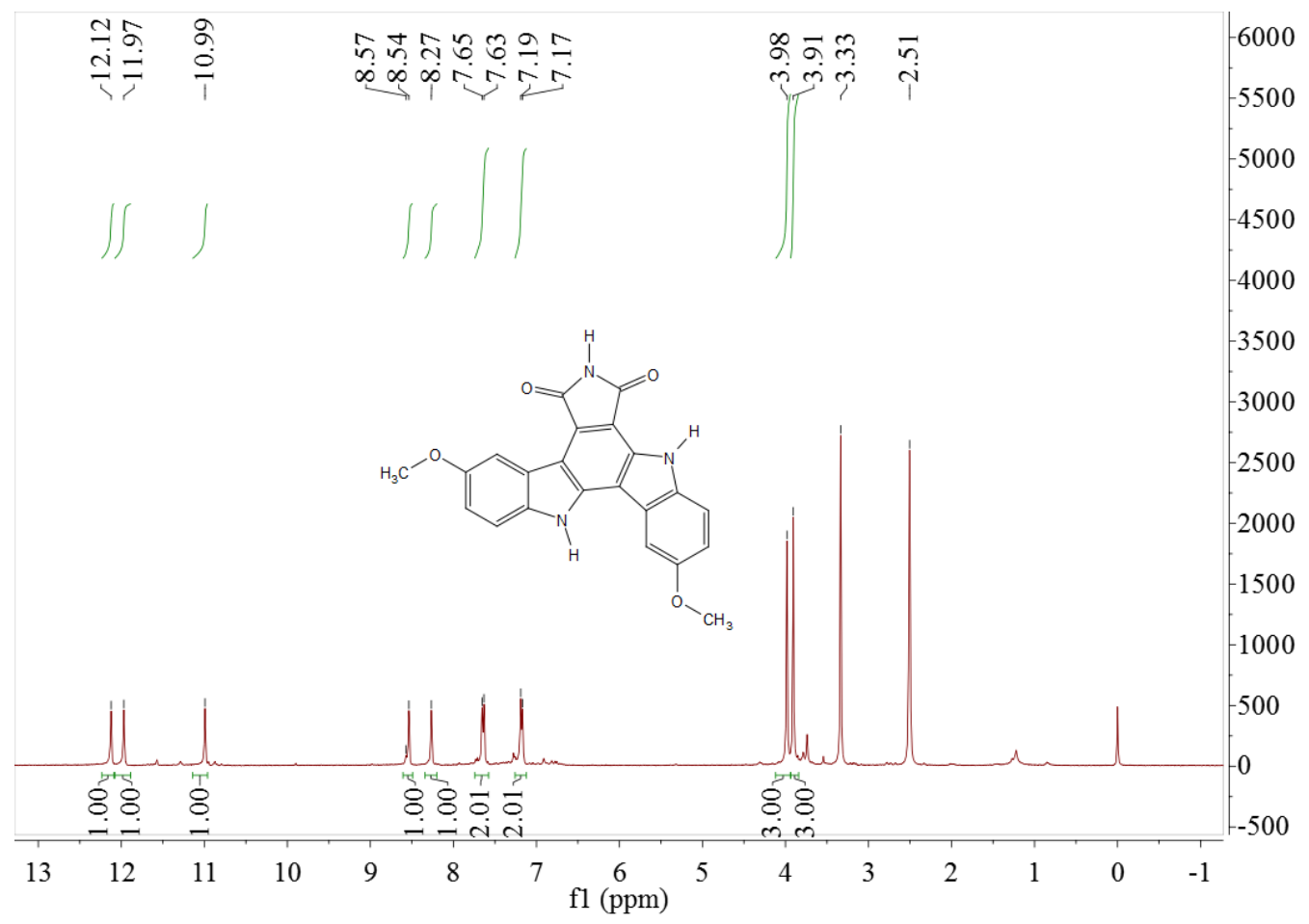

${ }^{1} \mathrm{H}$ NMR spectrum of 2,10-dimethoxy-5H-indolo[3,2-a]pyrrolo[3,4-c]carbazole-6,8(7H,13H)-dione (3b)

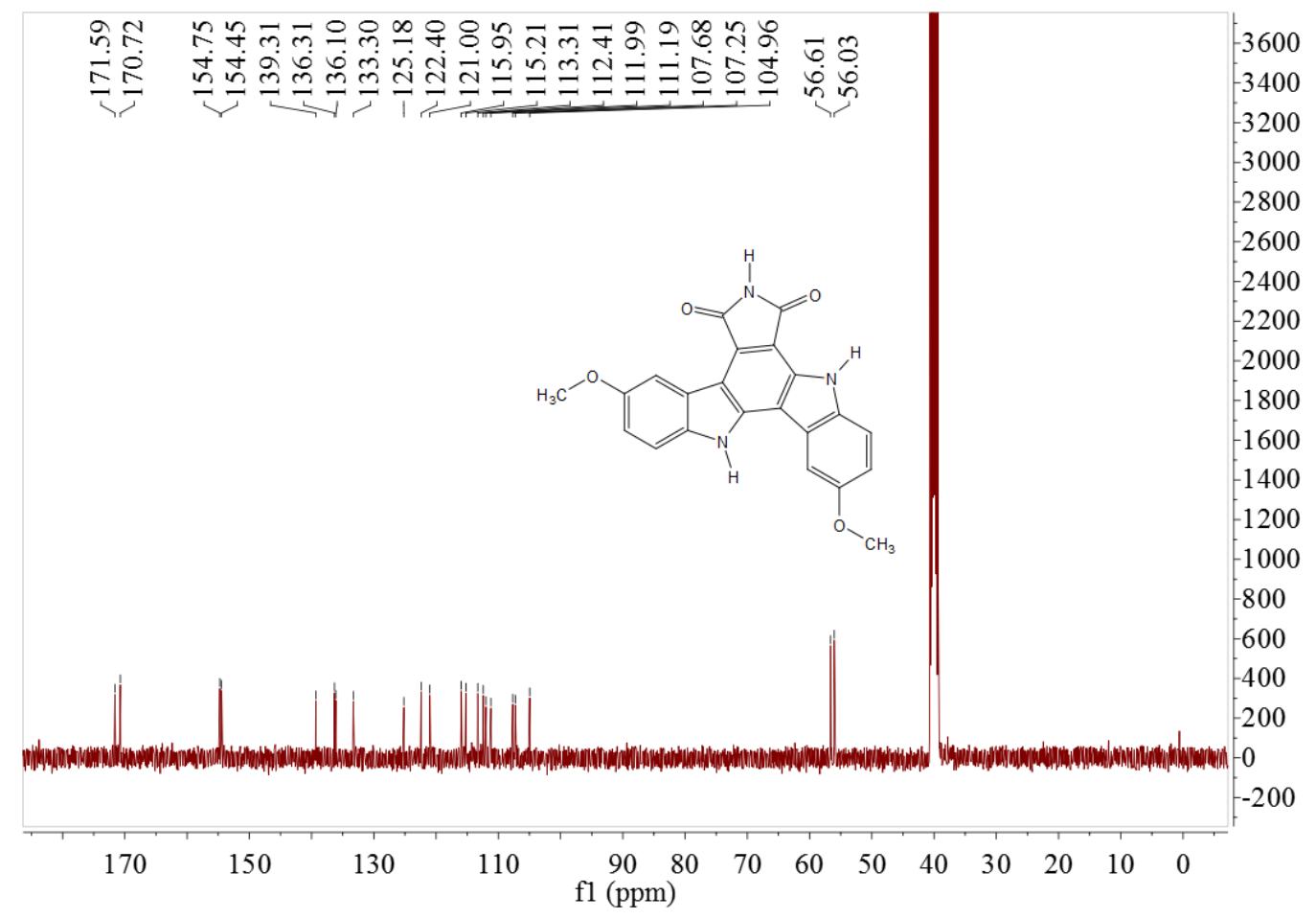

${ }^{13} \mathrm{C}$ NMR spectrum of 2,10-dimethoxy-5H-indolo[3,2-a]pyrrolo[3,4-c]carbazole-6,8(7H,13H)-dione (3b) 


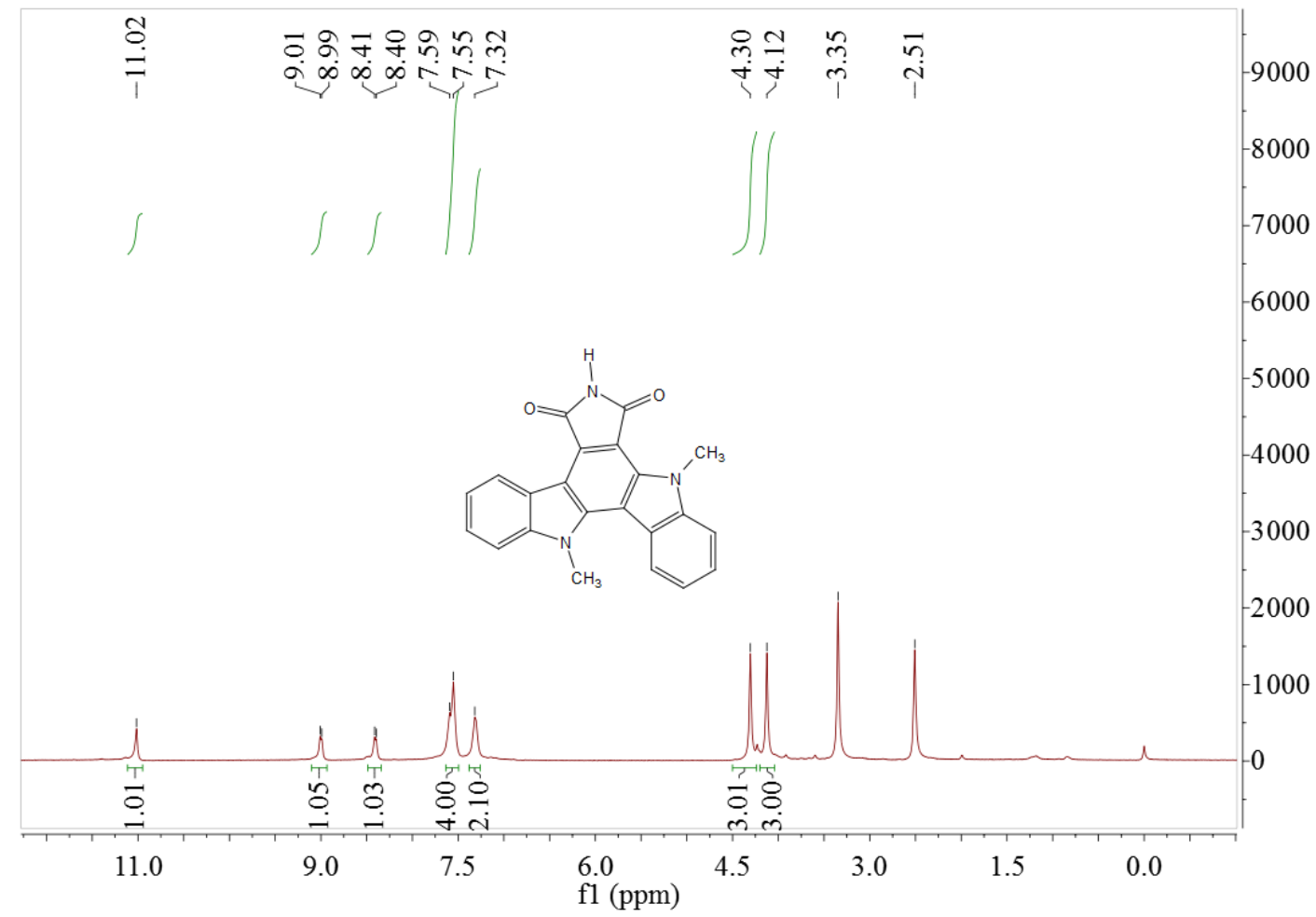

${ }^{1} \mathrm{H}$ NMR spectrum of 5,13-dimethyl-5H-indolo[3,2-a]pyrrolo[3,4-c]carbazole-6,8(7H,13H)-dione (3c)

ติ

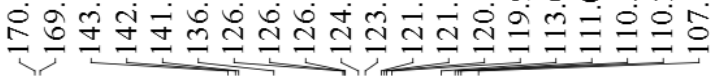

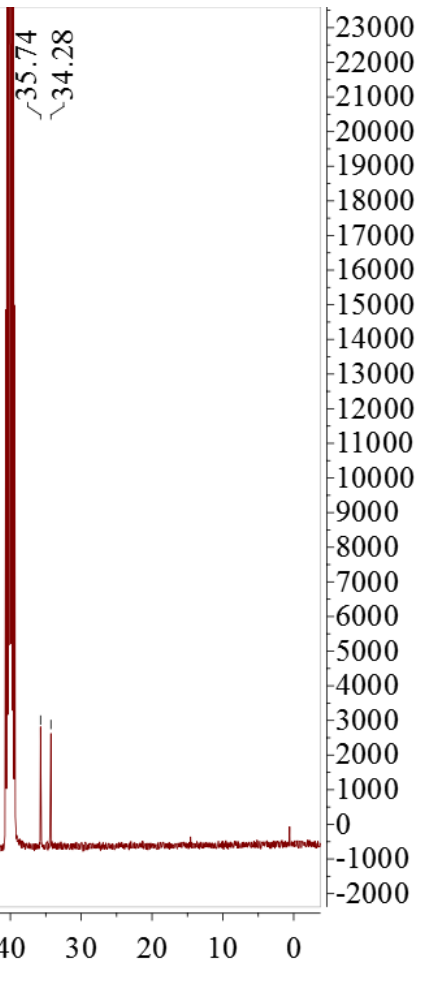

${ }^{13} \mathrm{C}$ NMR spectrum of 5,13-dimethyl-5H-indolo[3,2-a]pyrrolo[3,4-c]carbazole-6,8(7H,13H)-dione (3c) 


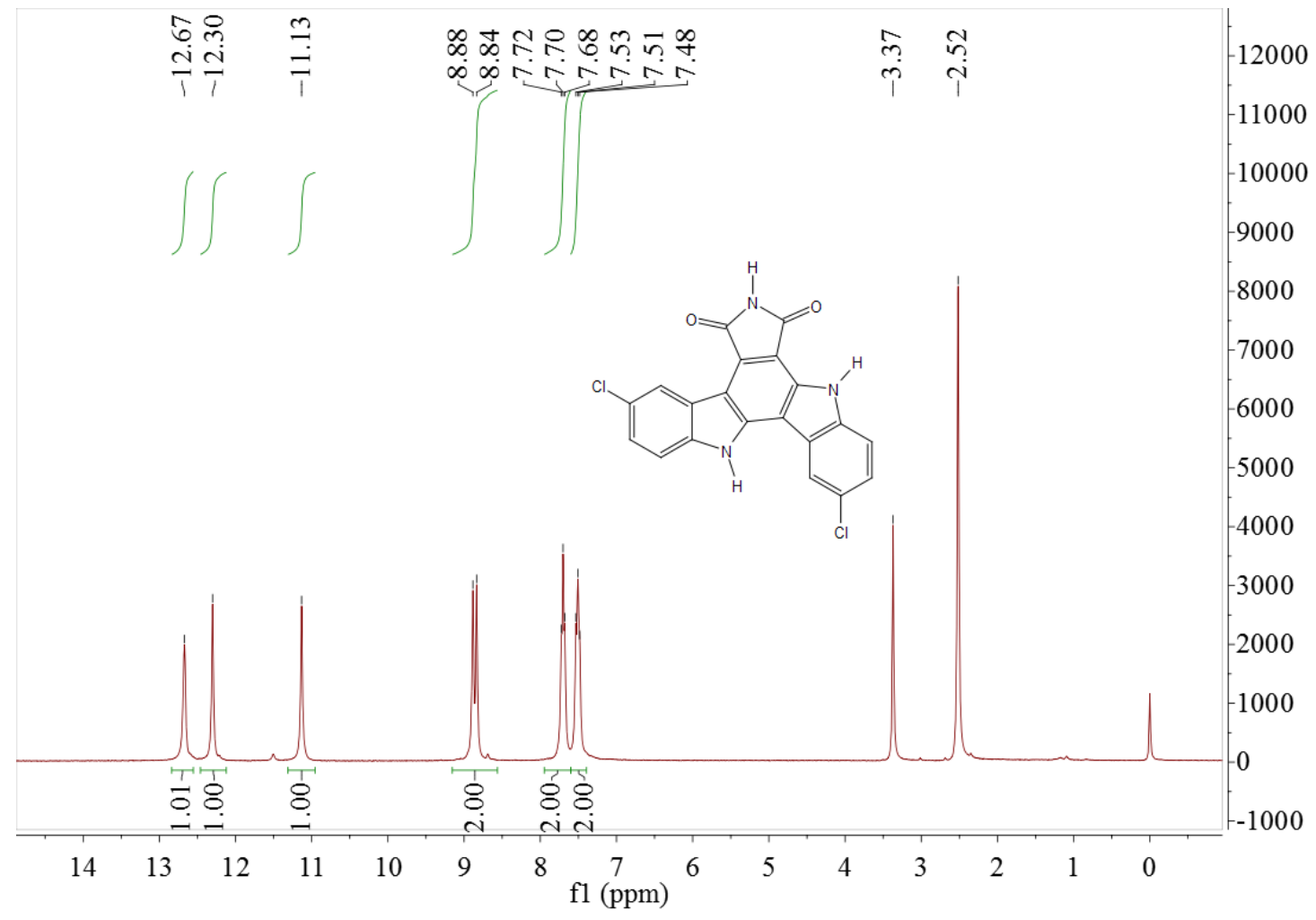

${ }^{1}$ H NMR spectrum of 2,10-dichloro-5H-indolo[3,2-a]pyrrolo[3,4-c]carbazole-6,8(7H,13H)-dione (3d)

송ำ

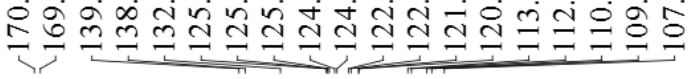

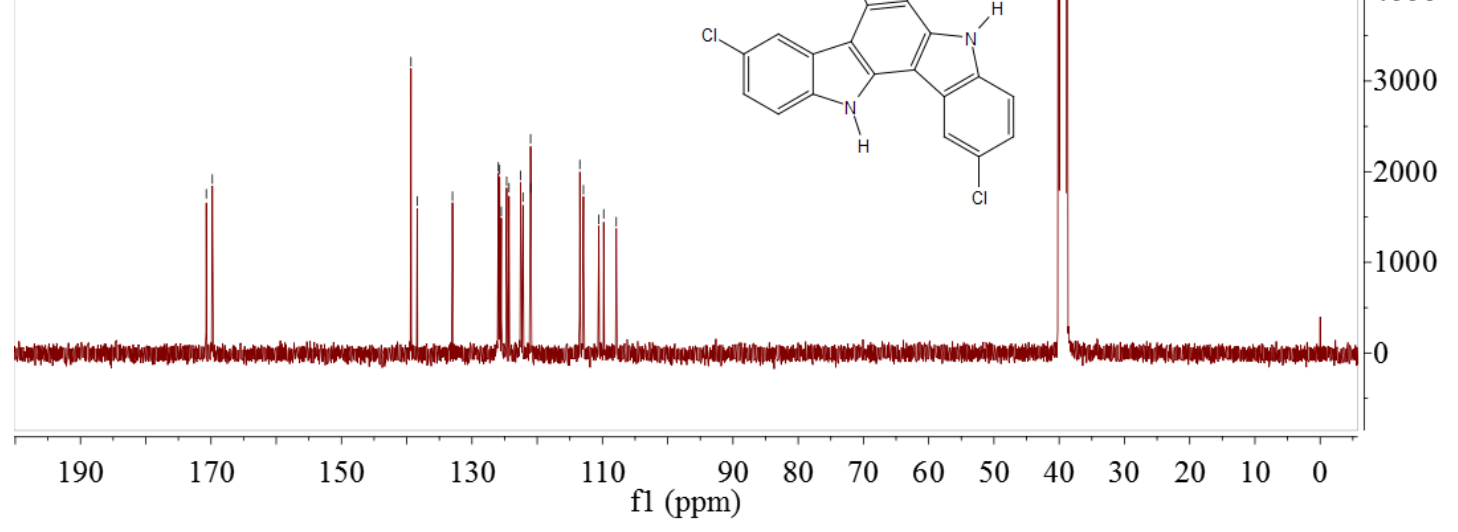

${ }^{13} \mathrm{C}$ NMR spectrum of 2,10-dichloro-5H-indolo[3,2-a]pyrrolo[3,4-c]carbazole-6,8(7H,13H)-dione (3d) 


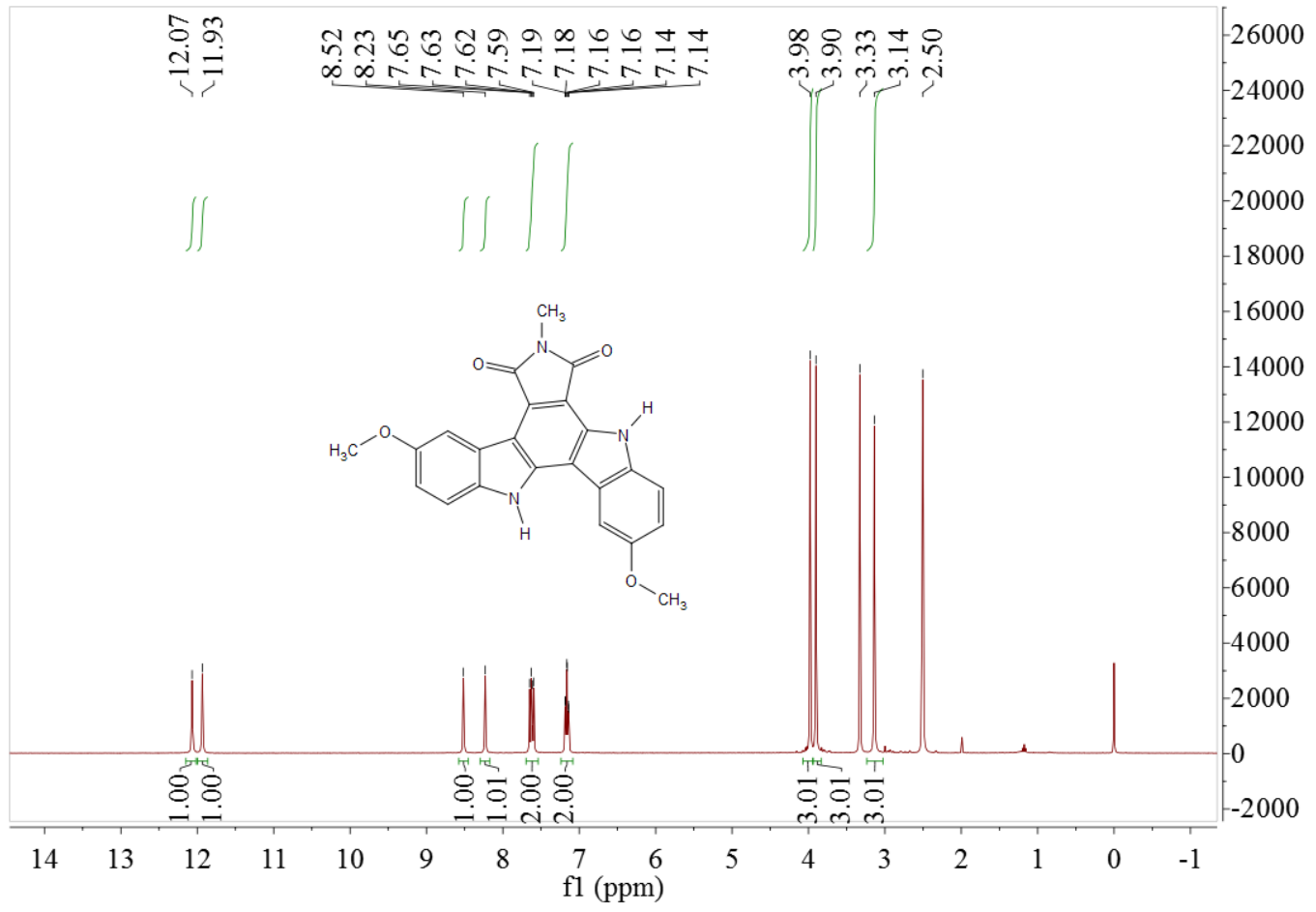

${ }^{1} \mathrm{H}$ NMR spectrum of 2,10-dimethoxy-7-methyl-5H-indolo[3,2-a]pyrrolo[3,4-c]carbazole-6,8(7H,13H) -dione (3e)
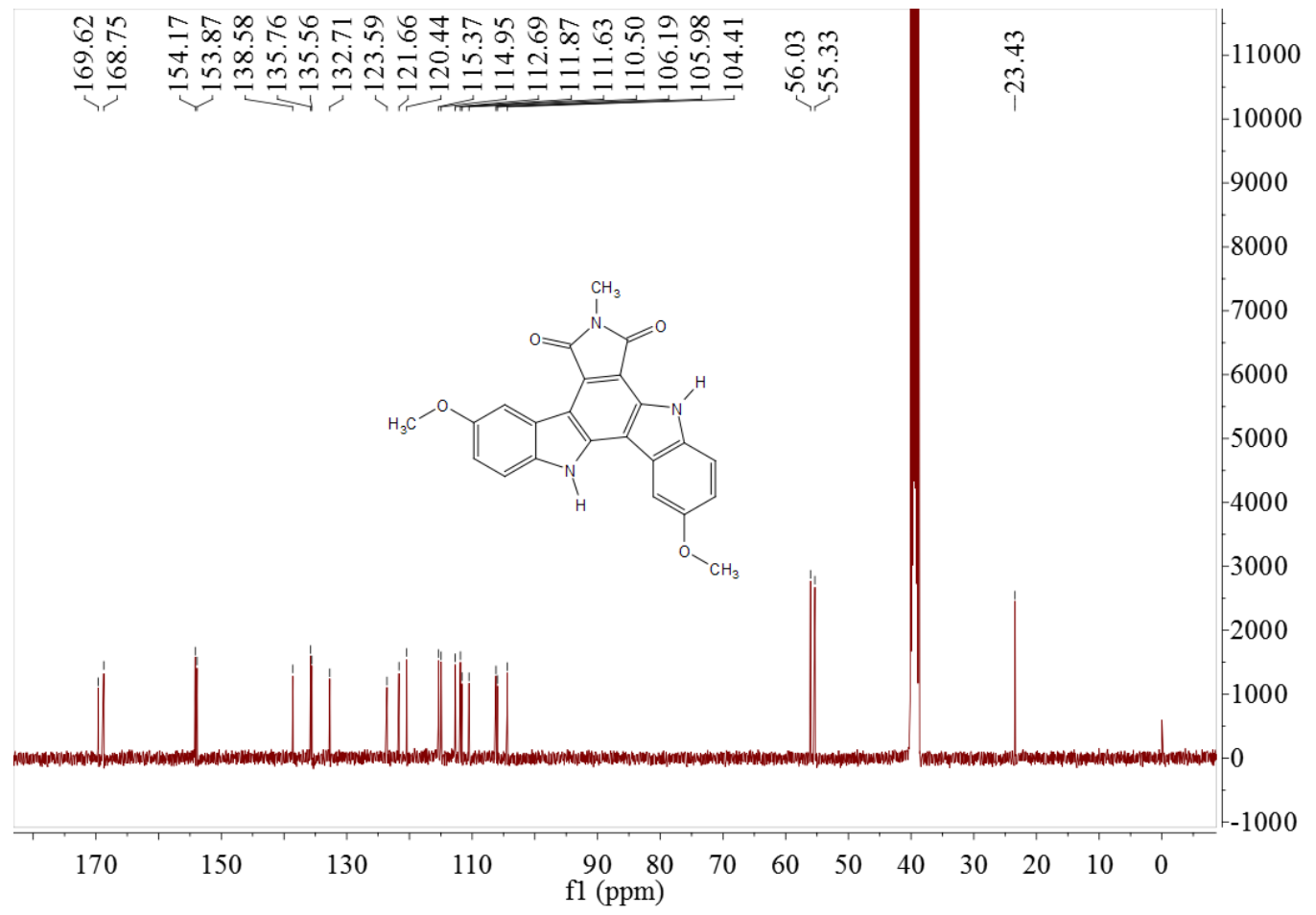

${ }^{13} \mathrm{C}$ NMR spectrum of 2,10-dimethoxy-7-methyl-5H-indolo[3,2-a]pyrrolo[3,4-c]carbazole-6,8(7H,13H) -dione (3e) 


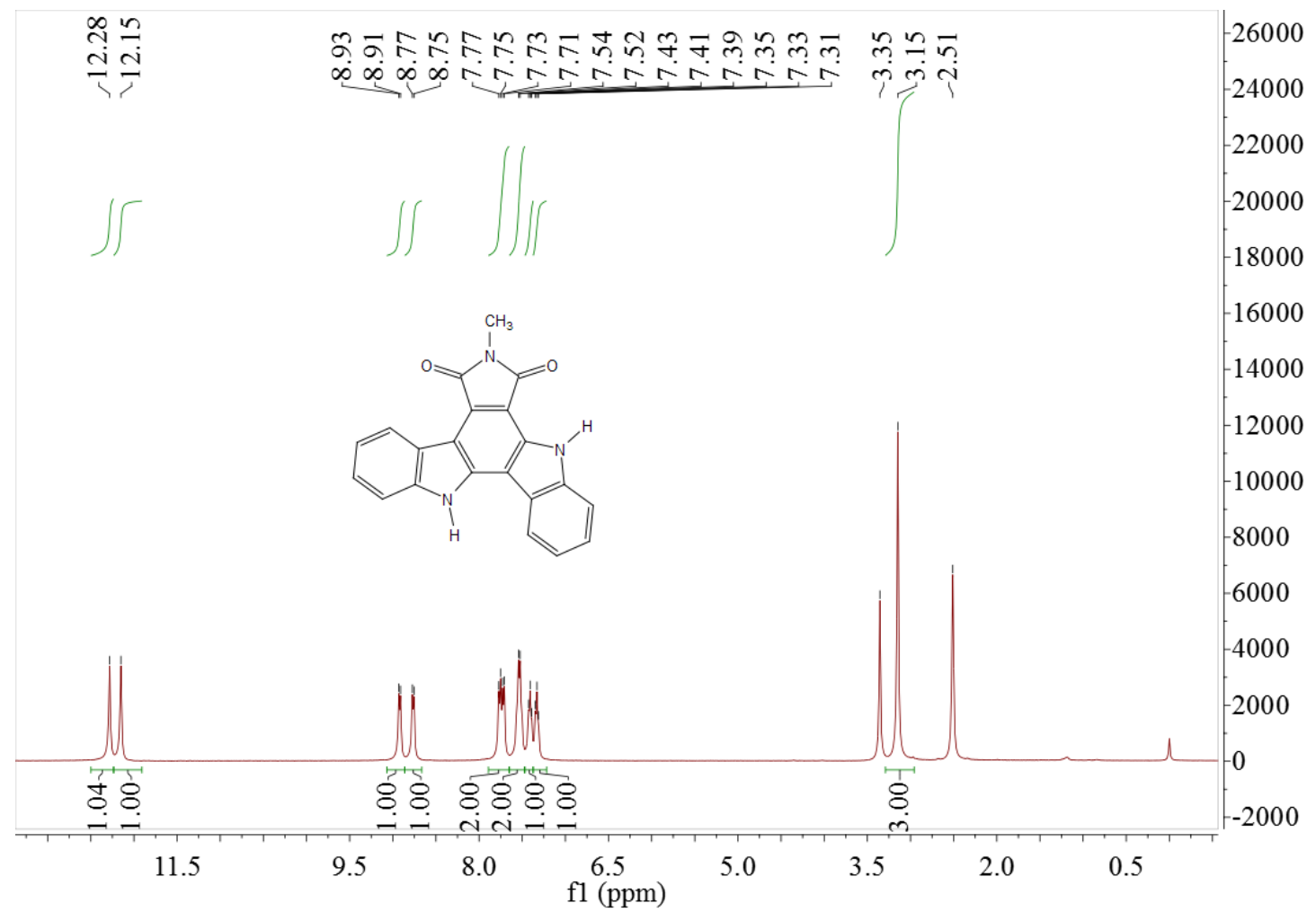

${ }^{1}$ H NMR spectrum of 7-methyl-5H-indolo[3,2-a]pyrrolo[3,4-c]carbazole-6,8(7H,13H)-dione (3f)

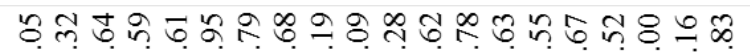

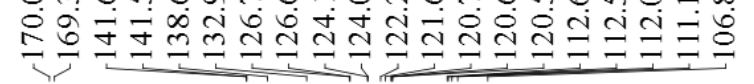

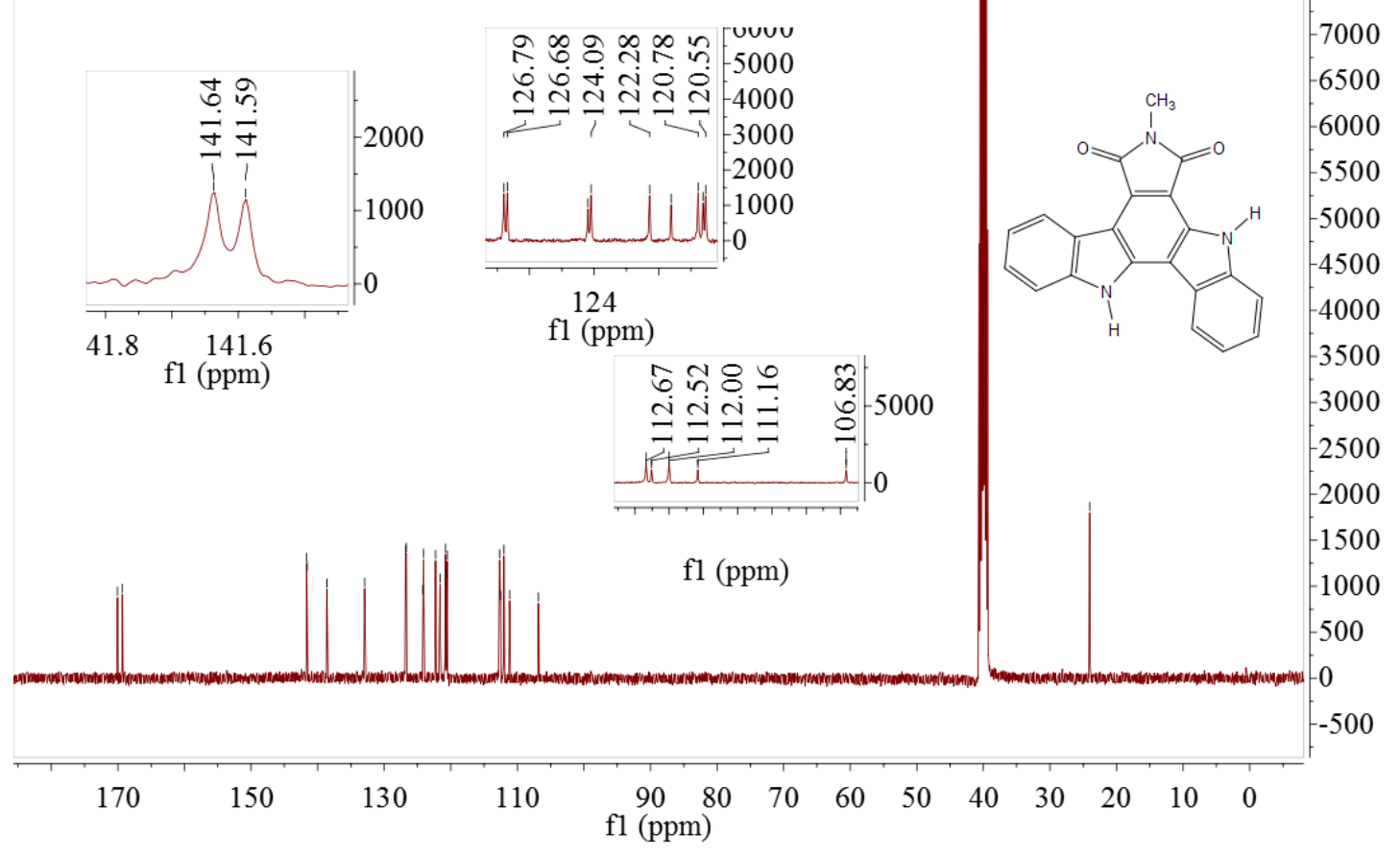

${ }^{13} \mathrm{C}$ NMR spectrum of 7-methyl-5H-indolo[3,2-a]pyrrolo[3,4-c]carbazole-6,8(7H,13H)-dione (3f) 


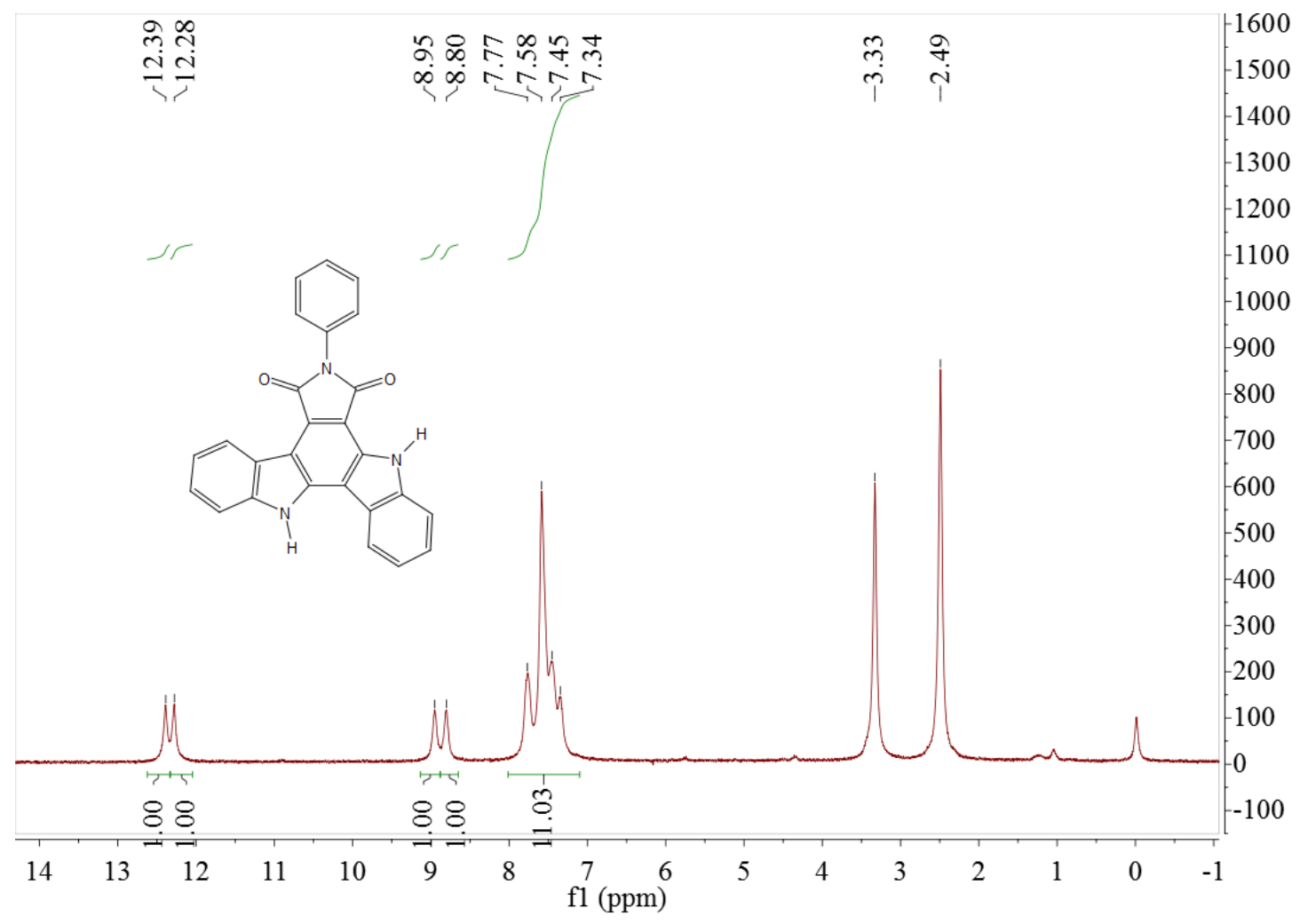

${ }^{1} \mathrm{H}$ NMR spectrum of 7-phenyl-5H-indolo[3,2-a]pyrrolo[3,4-c]carbazole-6,8(7H,13H)-dione (3g)

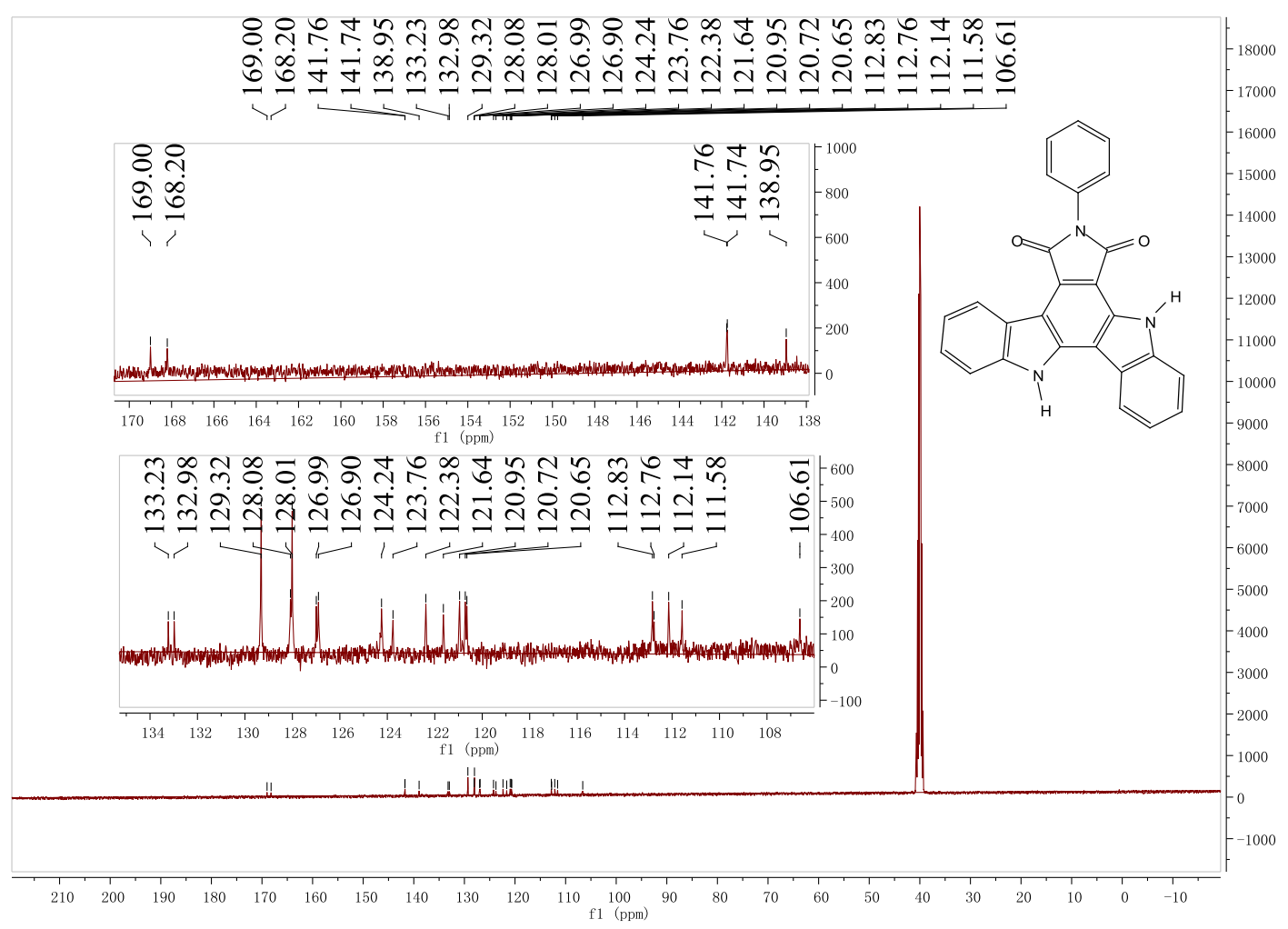

${ }^{13} \mathrm{C}$ NMR spectrum of 7-phenyl-5H-indolo[3,2-a]pyrrolo[3,4-c]carbazole-6,8(7H,13H)-dione $(\mathbf{3 g})$ 


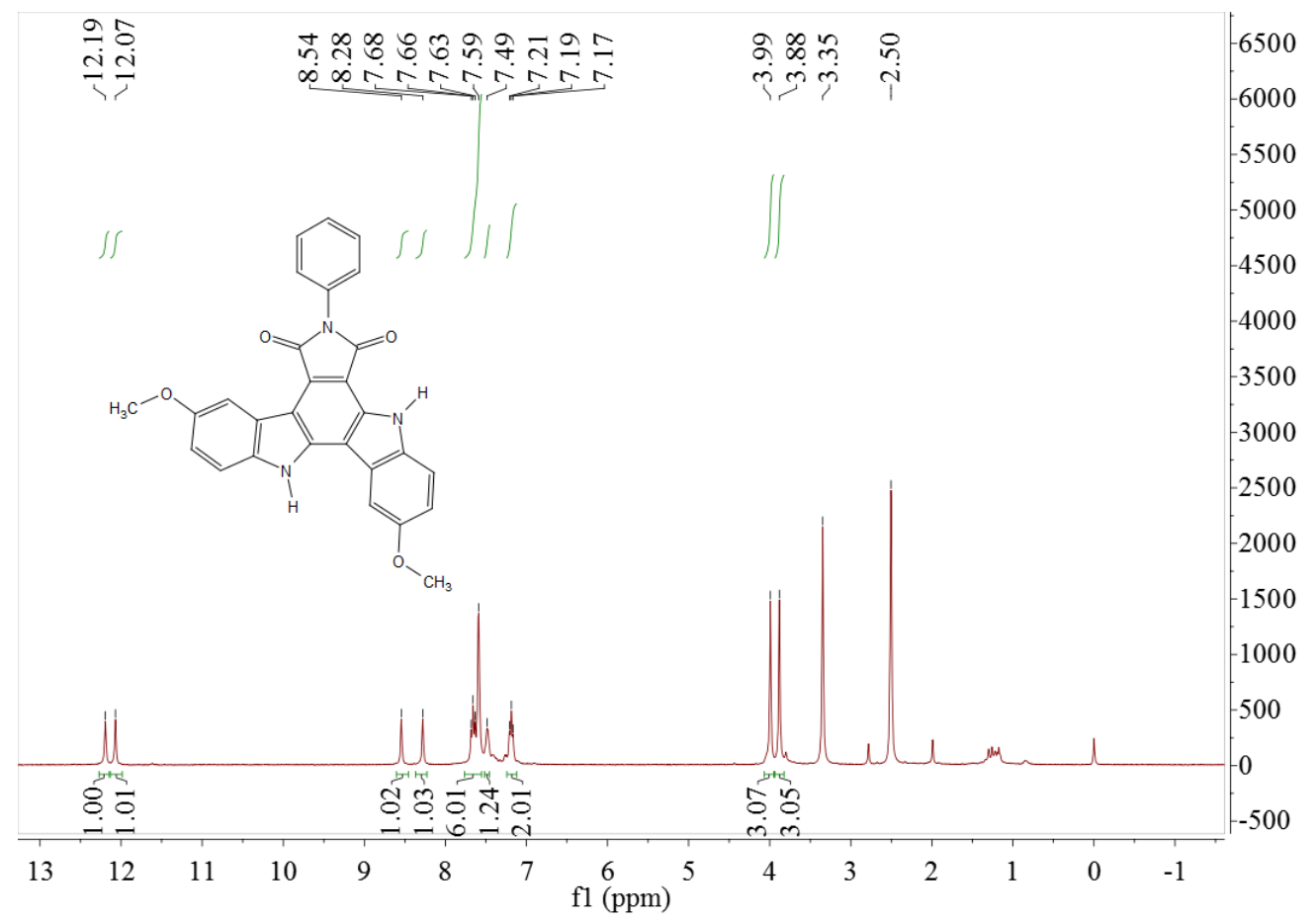

${ }^{1} \mathrm{H} \quad$ NMR $\quad$ spectrum of 2,10-dimethoxy-7-phenyl-5H-indolo[3,2-a]pyrrolo[3,4-c]carbazole6,8(7H,13H)-dione (3h)

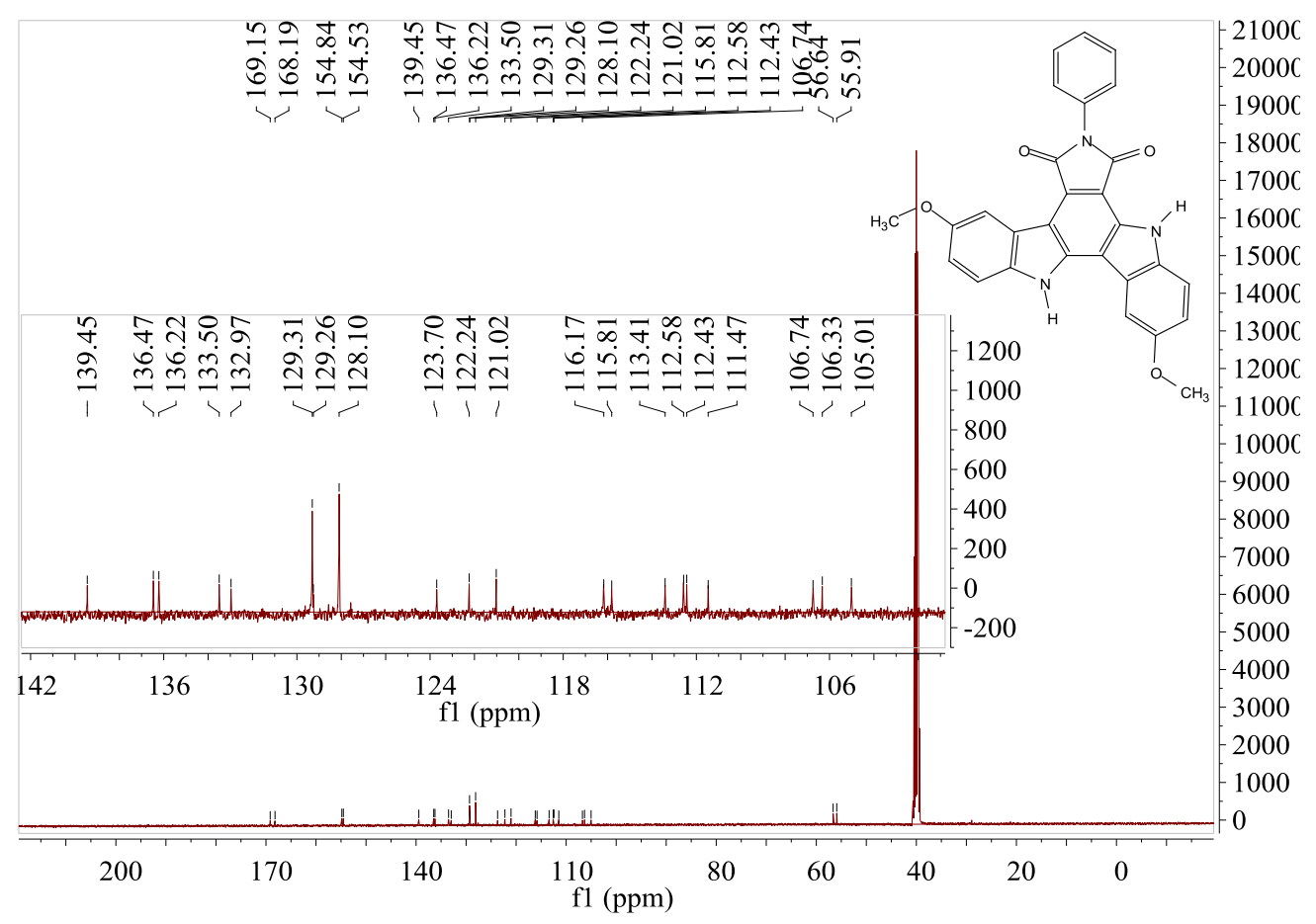

${ }^{13} \mathrm{C} \quad \mathrm{NMR}$ spectrum of 2,10-dimethoxy-7-phenyl-5H-indolo[3,2-a]pyrrolo[3,4-c]carbazole6,8(7H,13H)-dione (3h) 


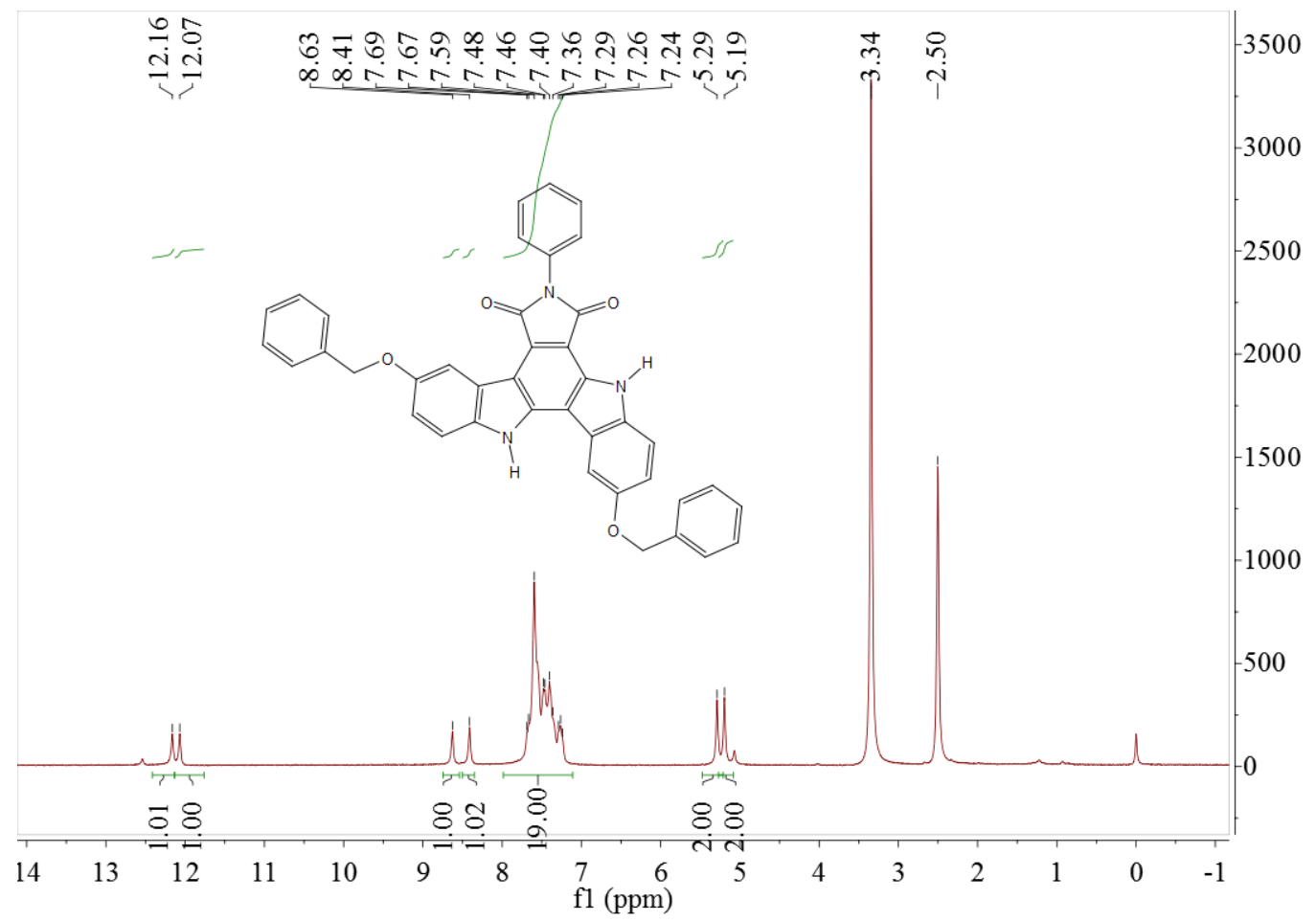

${ }^{1} \mathrm{H}$ NMR spectrum of 2,10-bis(benzyloxy)-7-phenyl-5H-indolo[3,2-a]pyrrolo[3,4-c]carbazole6,8(7H,13H)-dione (3i)

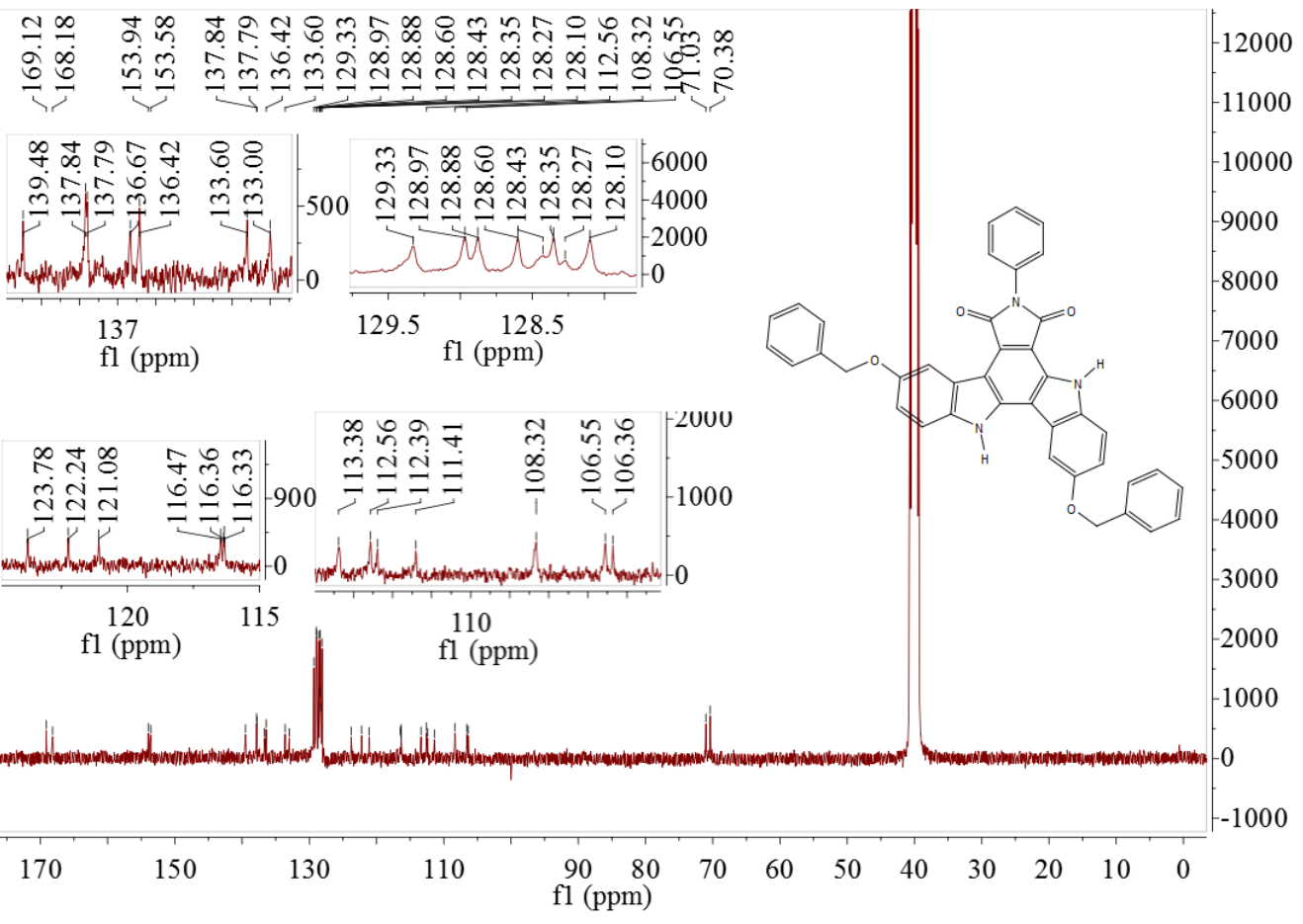

${ }^{13} \mathrm{C}$ NMR pectrum of 2,10-bis(benzyloxy)-7-phenyl-5H-indolo[3,2-a]pyrrolo[3,4-c]carbazole6,8(7H,13H)-dione (3i) 


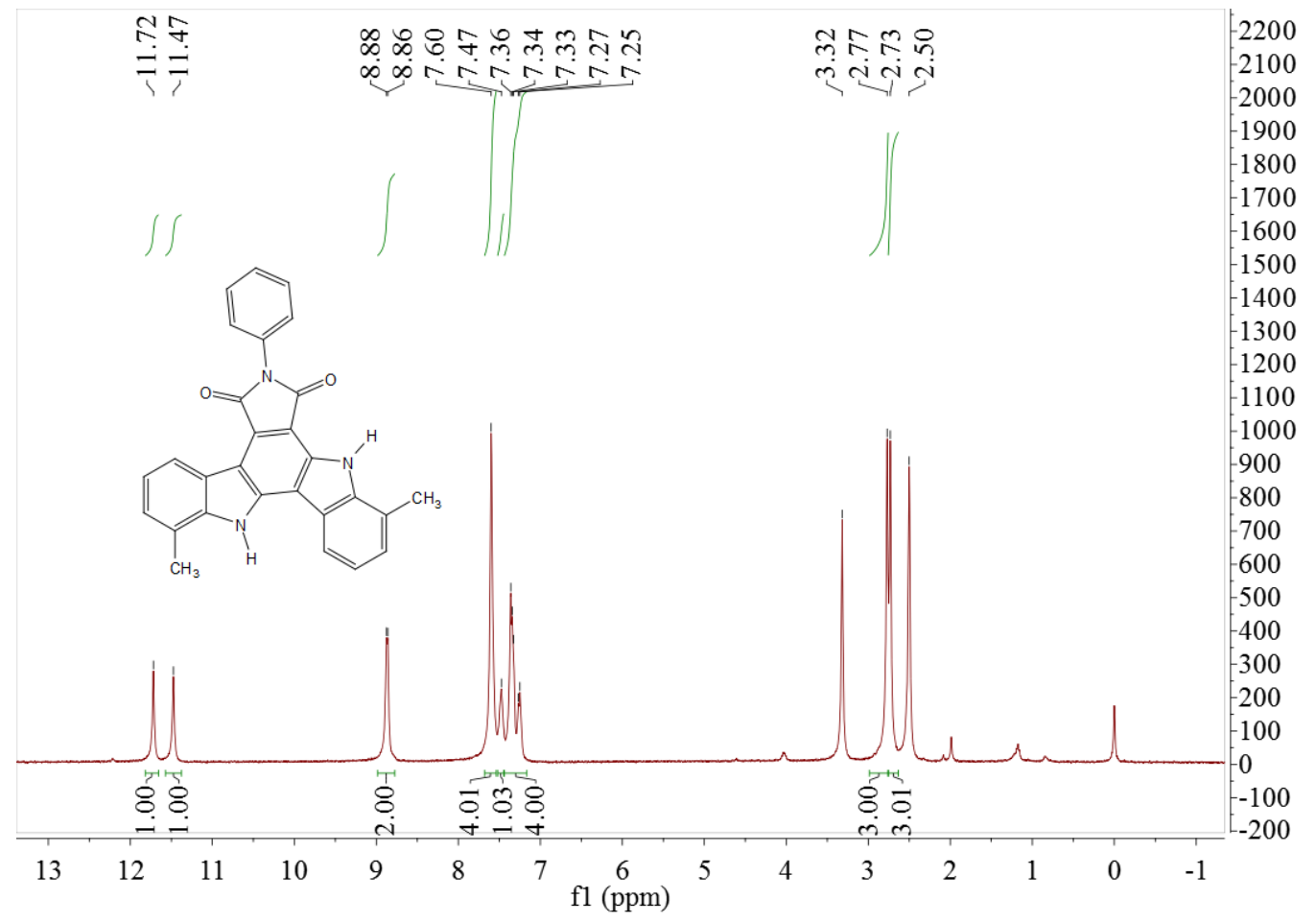

${ }^{1} \mathrm{H}$

NMR

spectrum

of

4,12-dimethyl-7-phenyl-5H-indolo[3,2-a]pyrrolo[3,4-c]carbazole-6,8(7H,13H)-dione (3j)

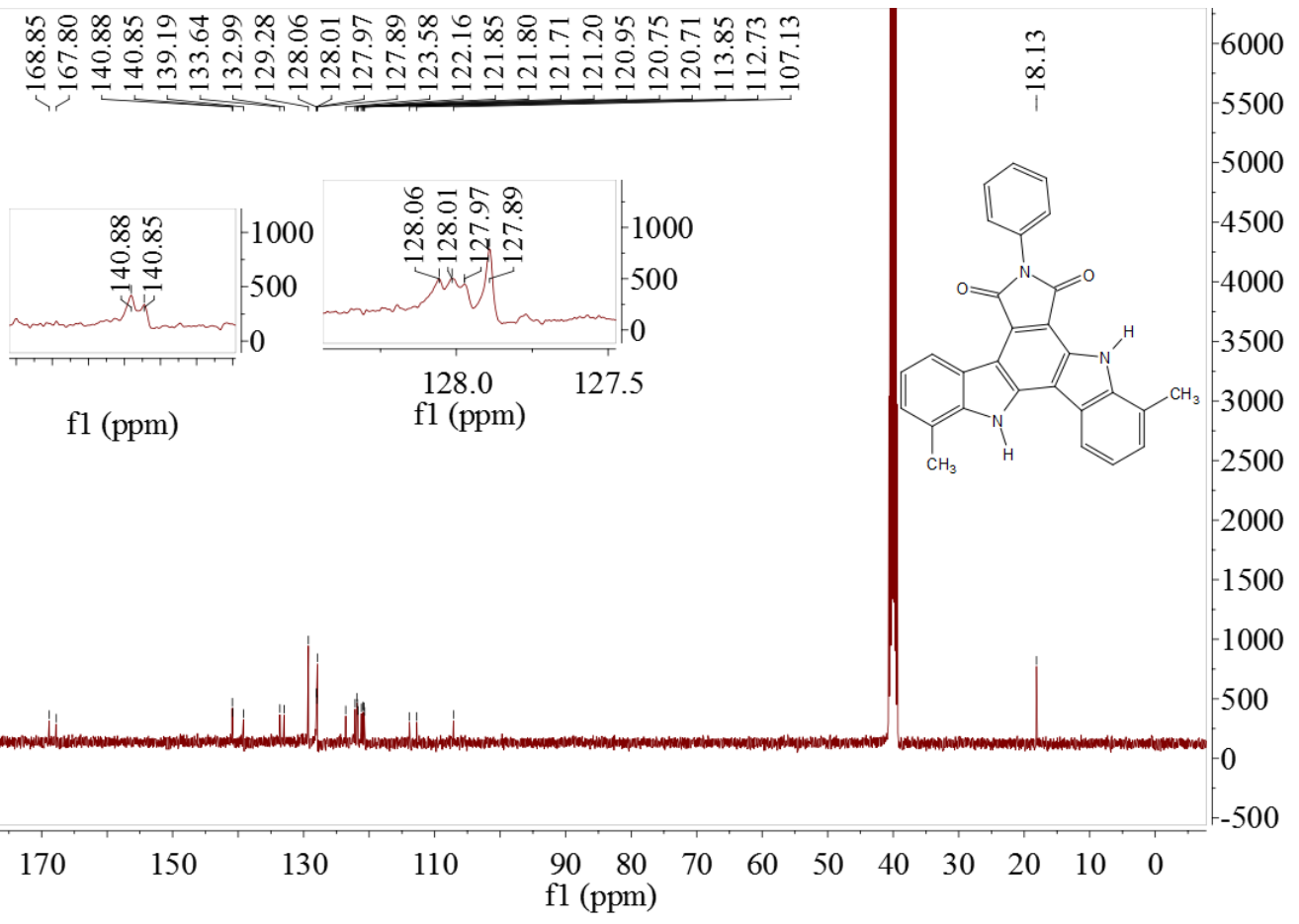

${ }^{13} \mathrm{C}$ NMR spectrum of 4,12-dimethyl-7-phenyl-5H-indolo[3,2-a]pyrrolo[3,4-c]carbazole- 6,8(7H,13H)dione (3j) 


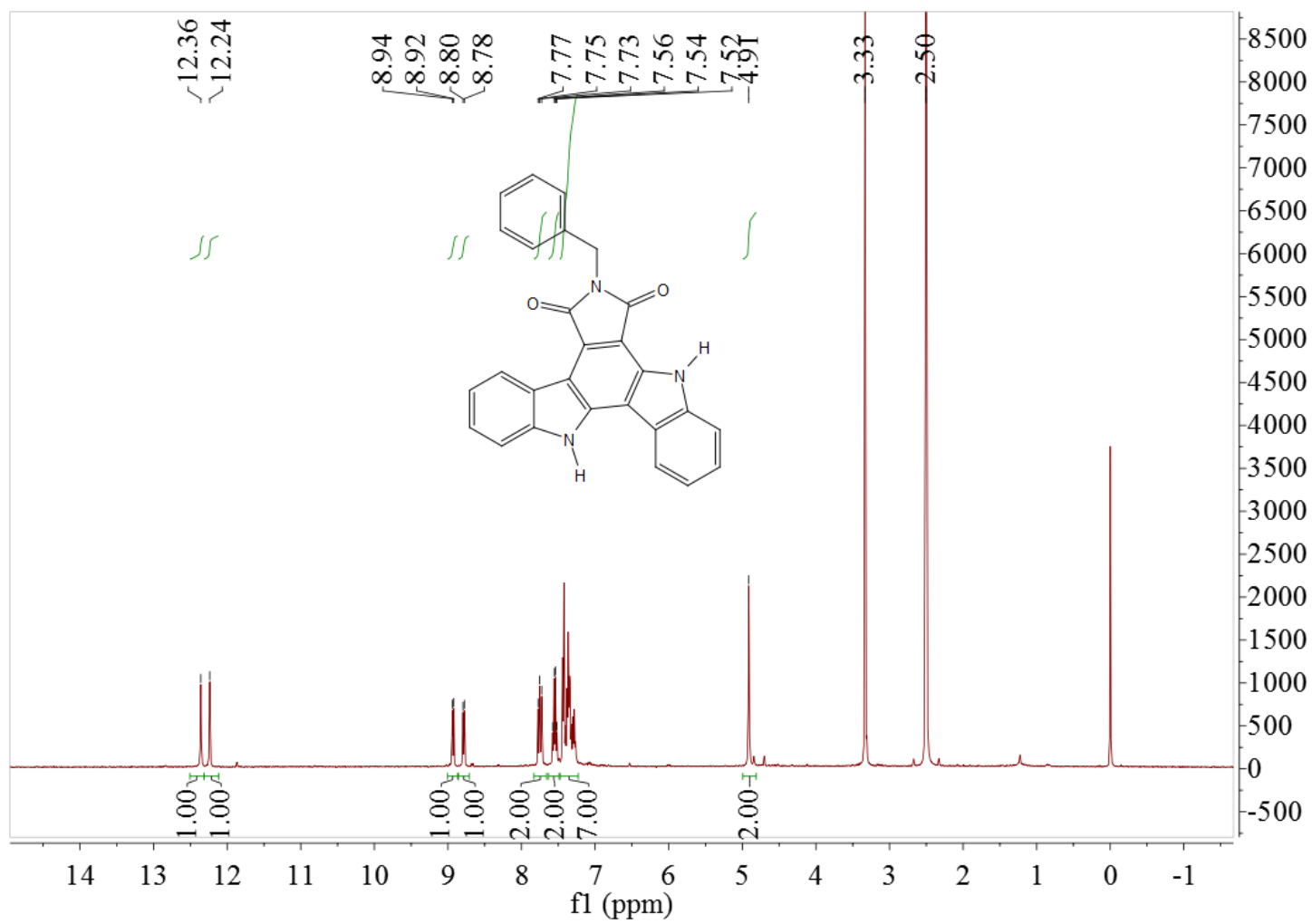

${ }^{1} \mathrm{H}$ NMR spectrum of 7-benzyl-5H-indolo[3,2-a]pyrrolo[3,4-c]carbazole-6,8(7H,13H)-dione (3k)

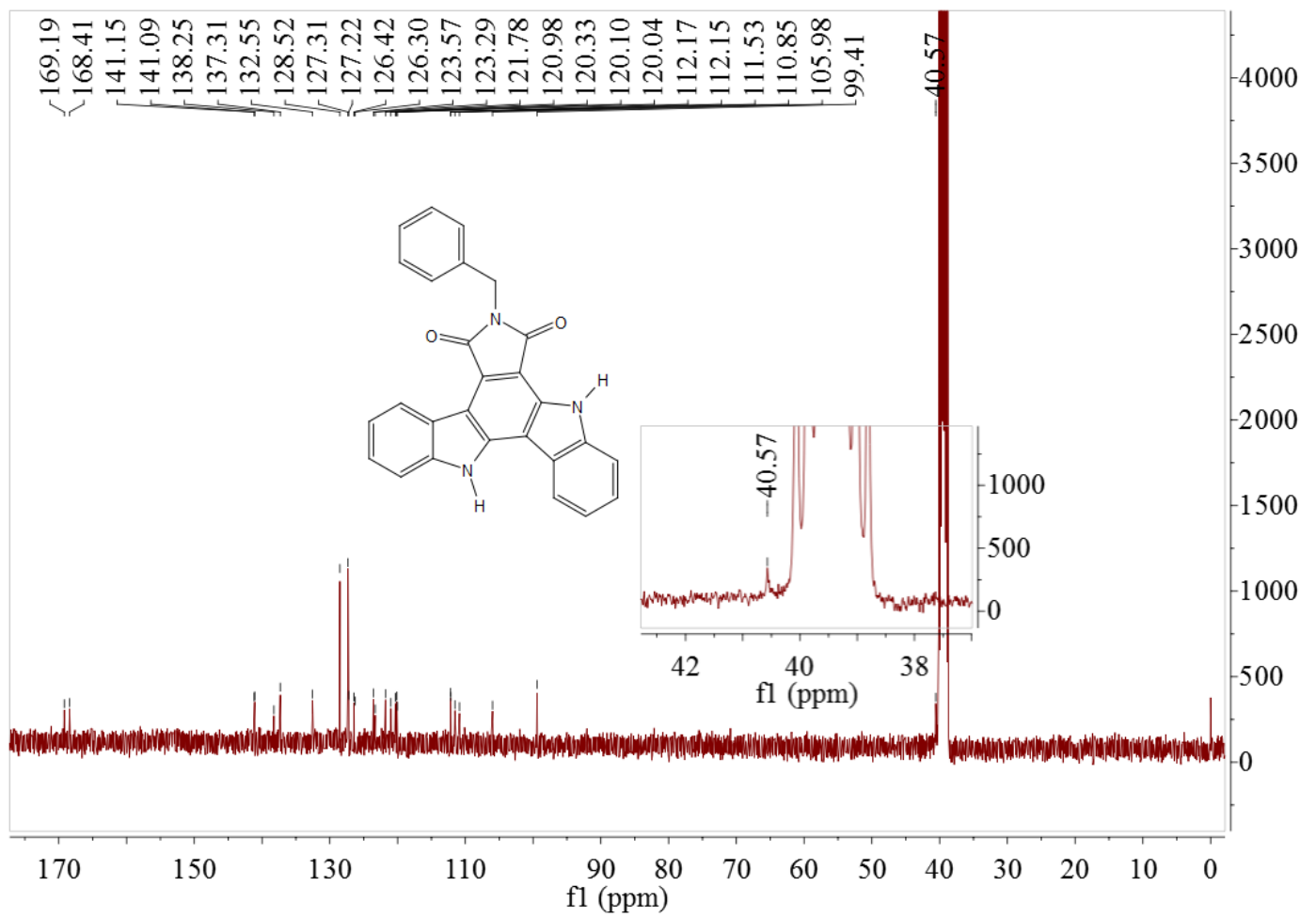

${ }^{13} \mathrm{C}$ NMR spectrum of 7-benzyl-5H-indolo[3,2-a]pyrrolo[3,4-c]carbazole-6,8(7H,13H)-dione (3k) 


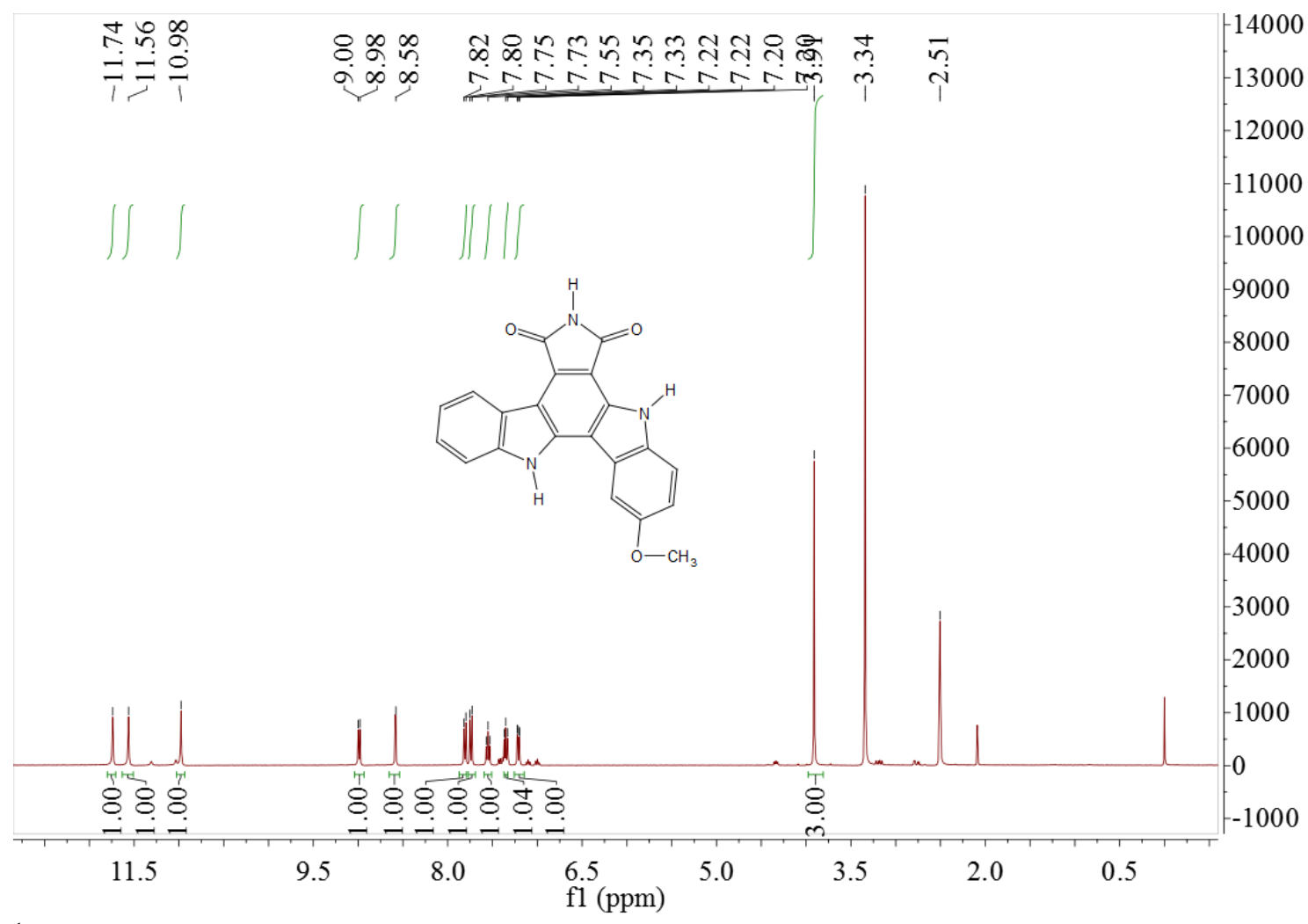

${ }^{1} \mathrm{H}$ NMR spectrum of 2-methoxy-5H-indolo[3,2-a]pyrrolo[3,4-c]carbazole-6,8(7H,13H)-dione (31)

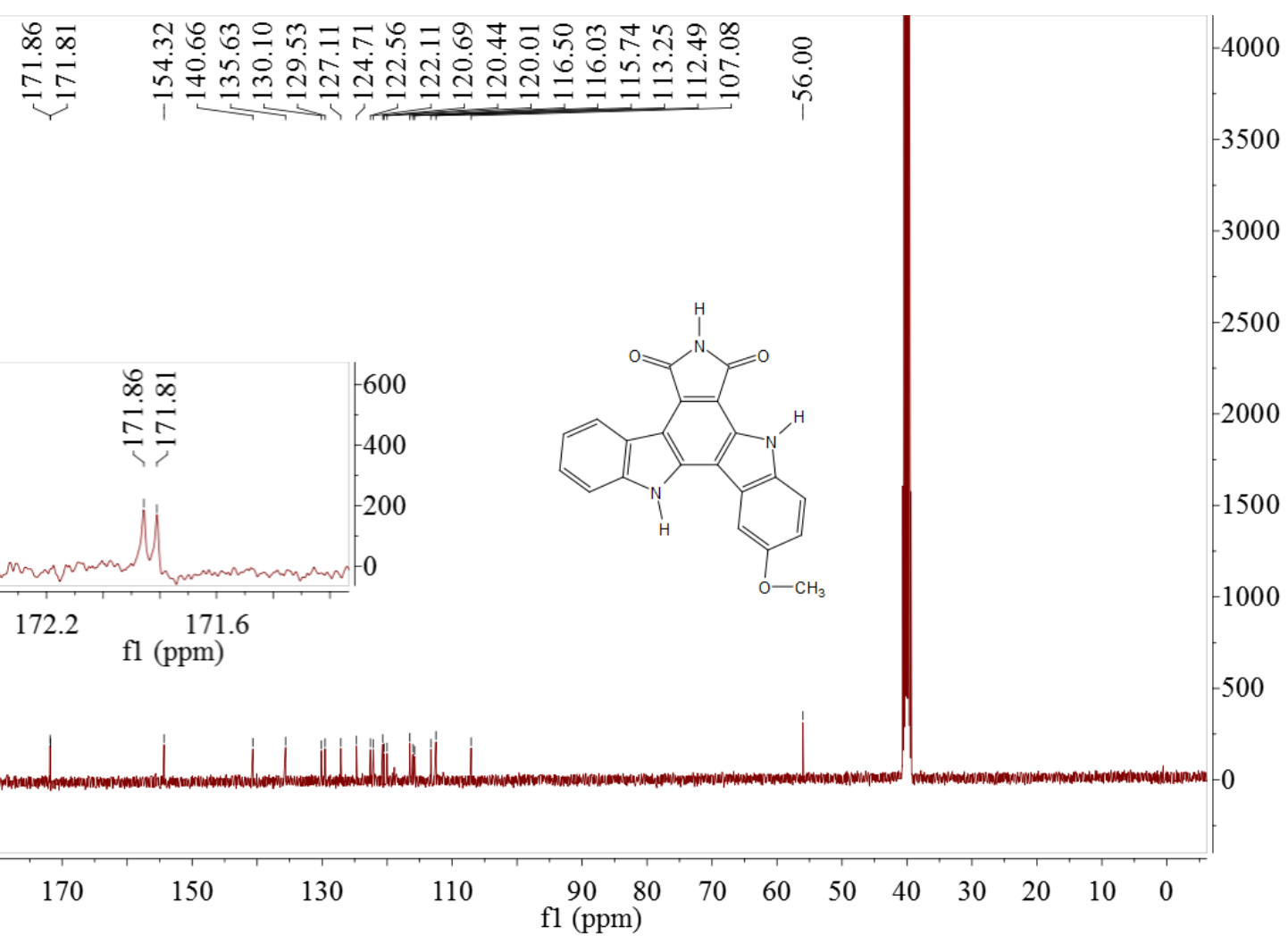

${ }^{13} \mathrm{C}$ NMR spectrum of 2-methoxy-5H-indolo[3,2-a]pyrrolo[3,4-c]carbazole-6,8(7H,13H)-dione (3I) 


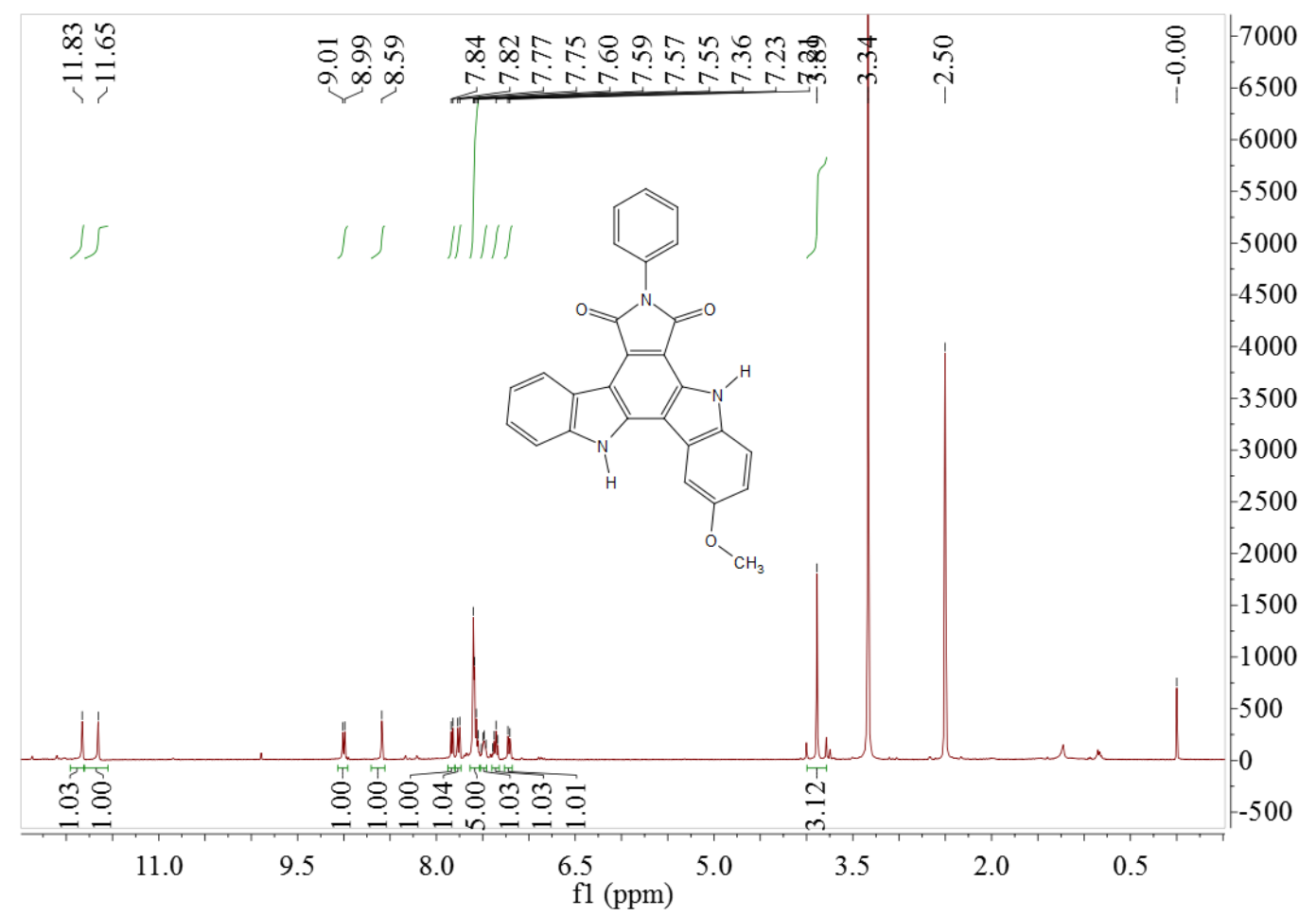

${ }^{1} \mathrm{H} \quad$ NMR $\quad$ spectrum of 2-methoxy-7-phenyl-5H-indolo[3,2-a]pyrrolo[3,4-c]carbazole6,8(7H,13H)-dione (3m)

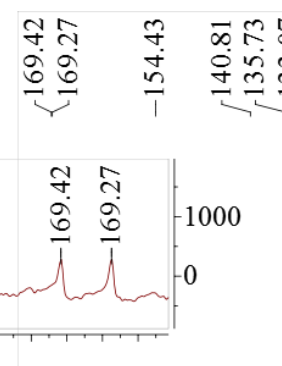

fl (ppm)
.

$\circ \sqrt[n]{\infty}$

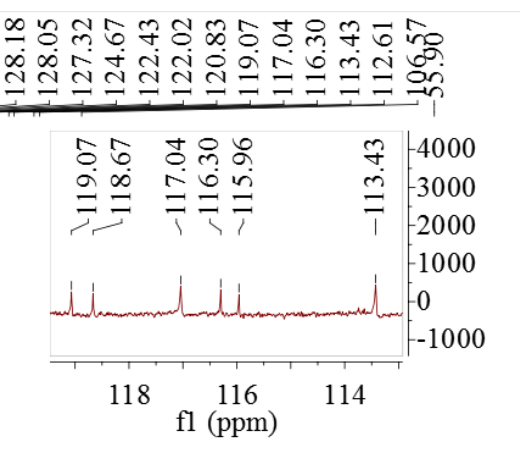

fl (ppm)

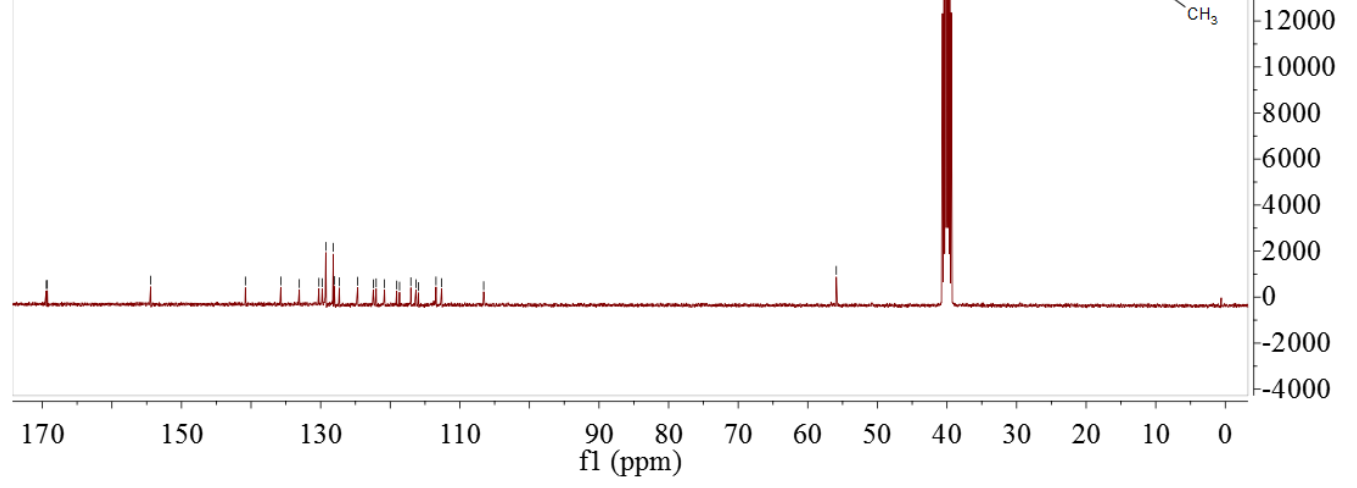

${ }^{13} \mathrm{C} \quad$ NMR spectrum of 2-methoxy-7-phenyl-5H-indolo[3,2-a]pyrrolo[3,4-c]carbazole6,8(7H,13H)-dione (3m) 


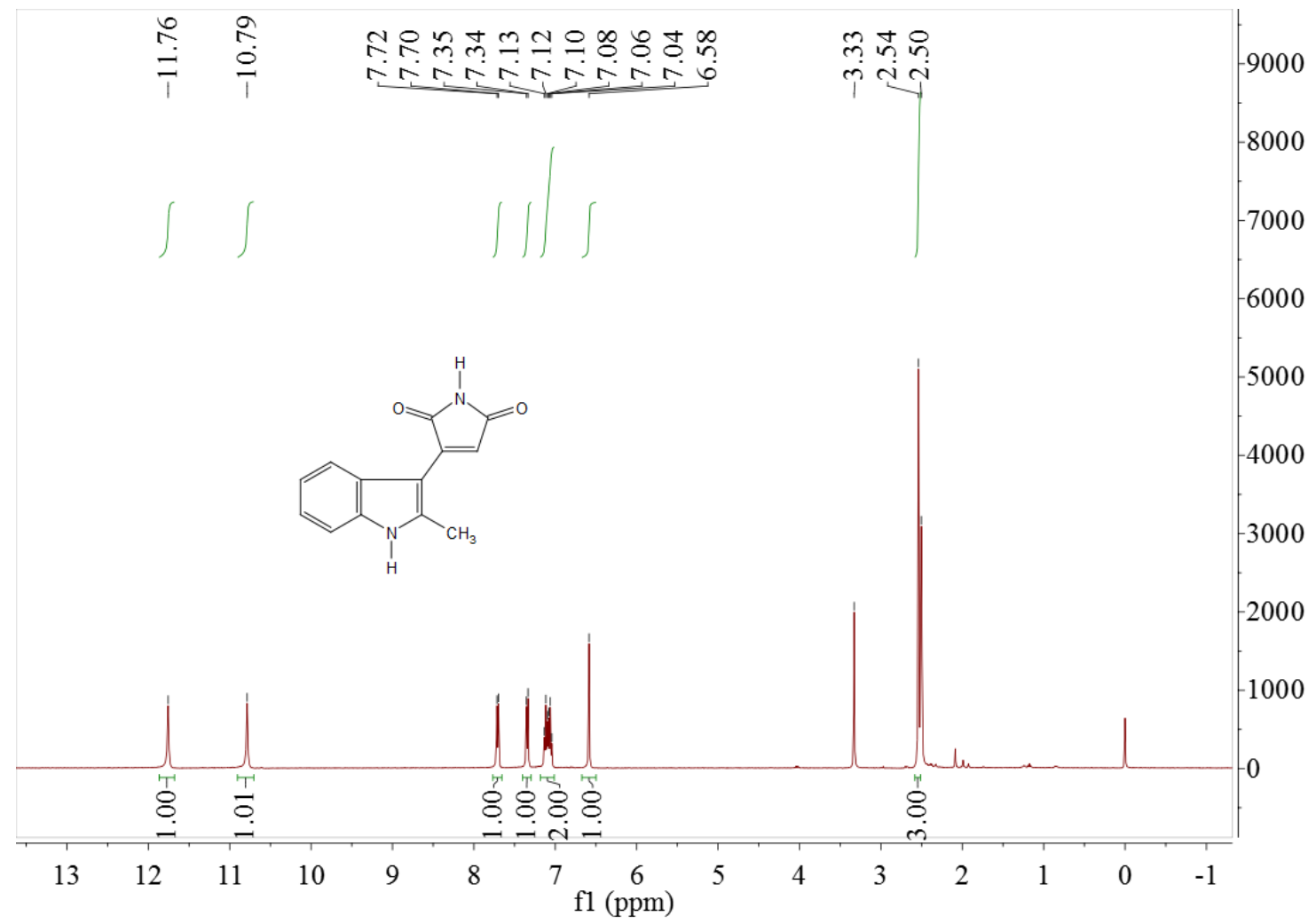

${ }^{1} \mathrm{H}$ NMR spectrum of 3-(2-methyl-1 $H$-indol-3-yl)-1 $H$-pyrrole-2,5-dione (4a)
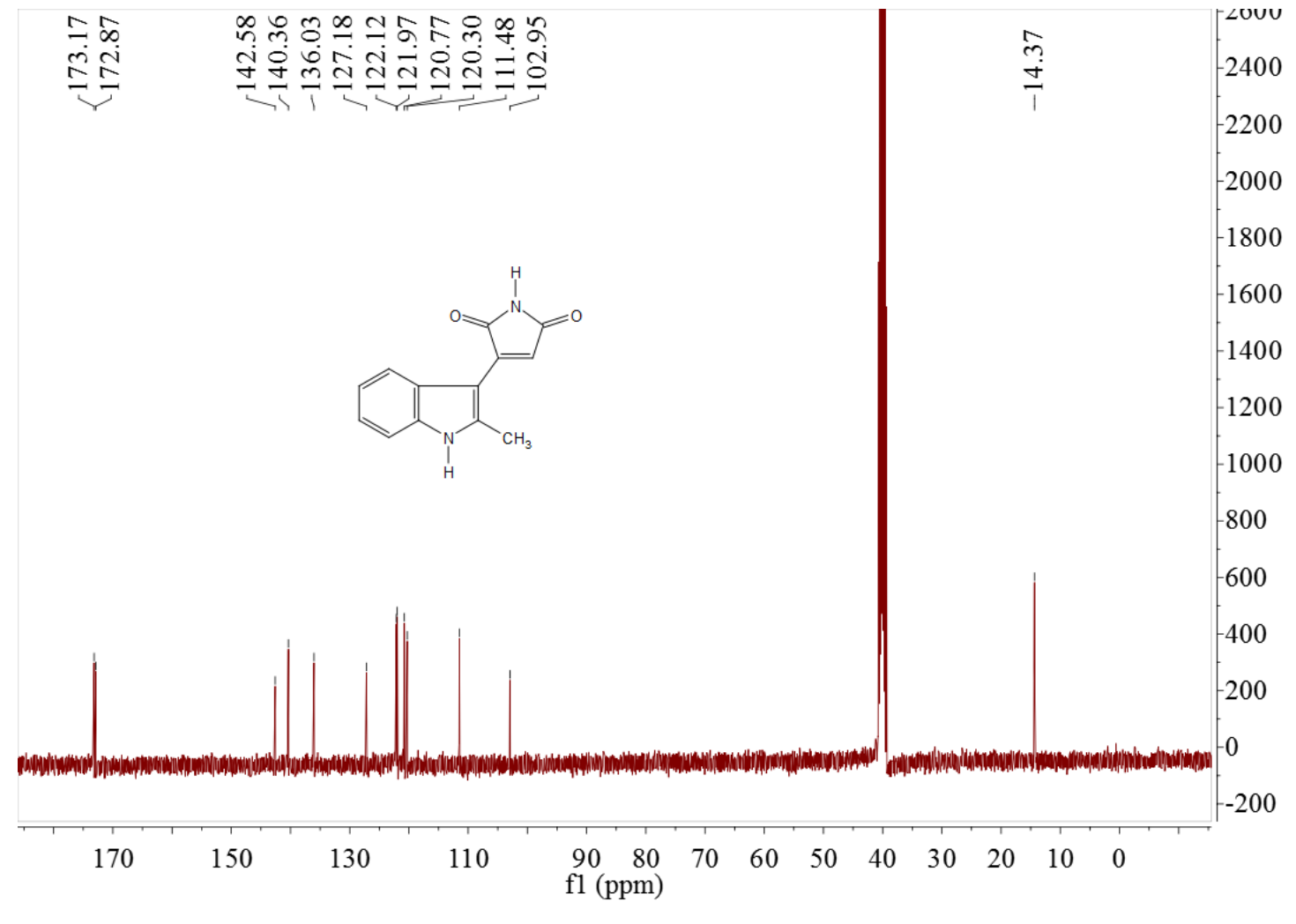

${ }^{13} \mathrm{C}$ NMR spectrum of 3-(2-methyl-1H-indol-3-yl)-1H-pyrrole-2,5-dione (4a) 


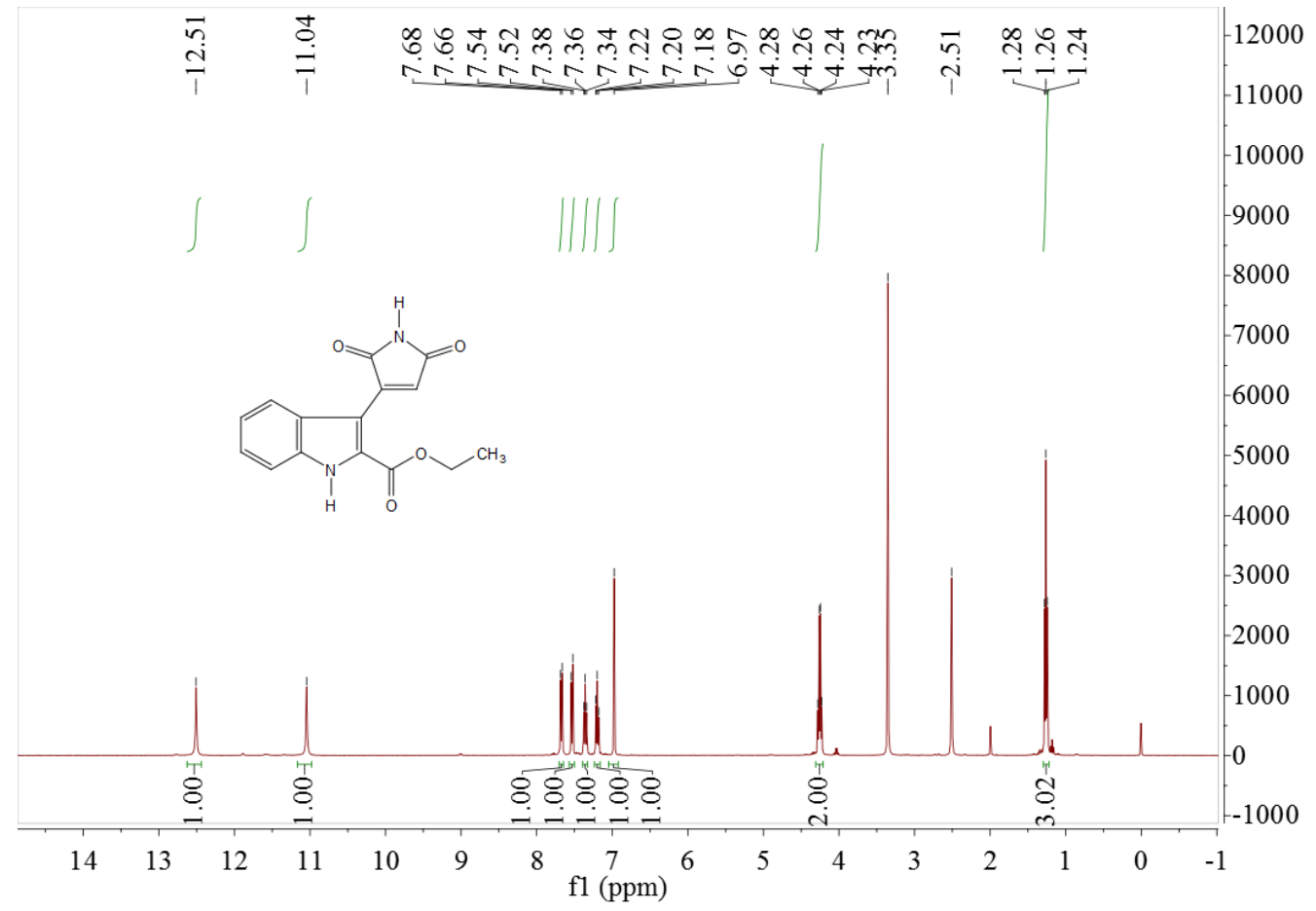

${ }^{1} \mathrm{H}$ NMR spectrum of 3-(2,5-dioxo-2,5-dihydro- $1 H$-pyrrol-3-yl)- $1 H$-indole-2-carboxylic acid ethyl ester (4b)

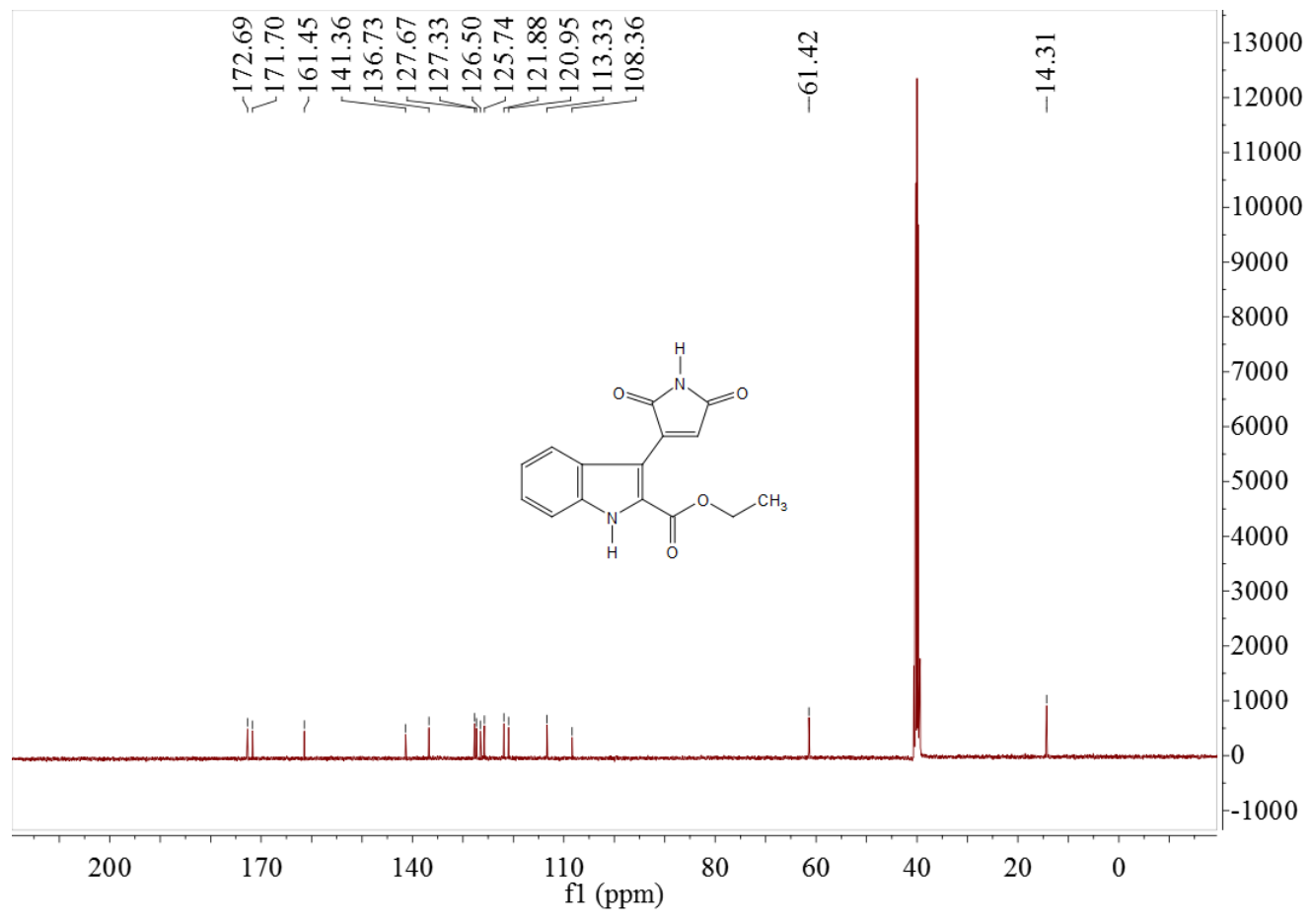

${ }^{13} \mathrm{C}$ NMR spectrum of 3-(2,5-dioxo-2,5-dihydro- $1 H$-pyrrol-3-yl)- $1 H$-indole-2-carboxylic acid ethyl ester (4b) 


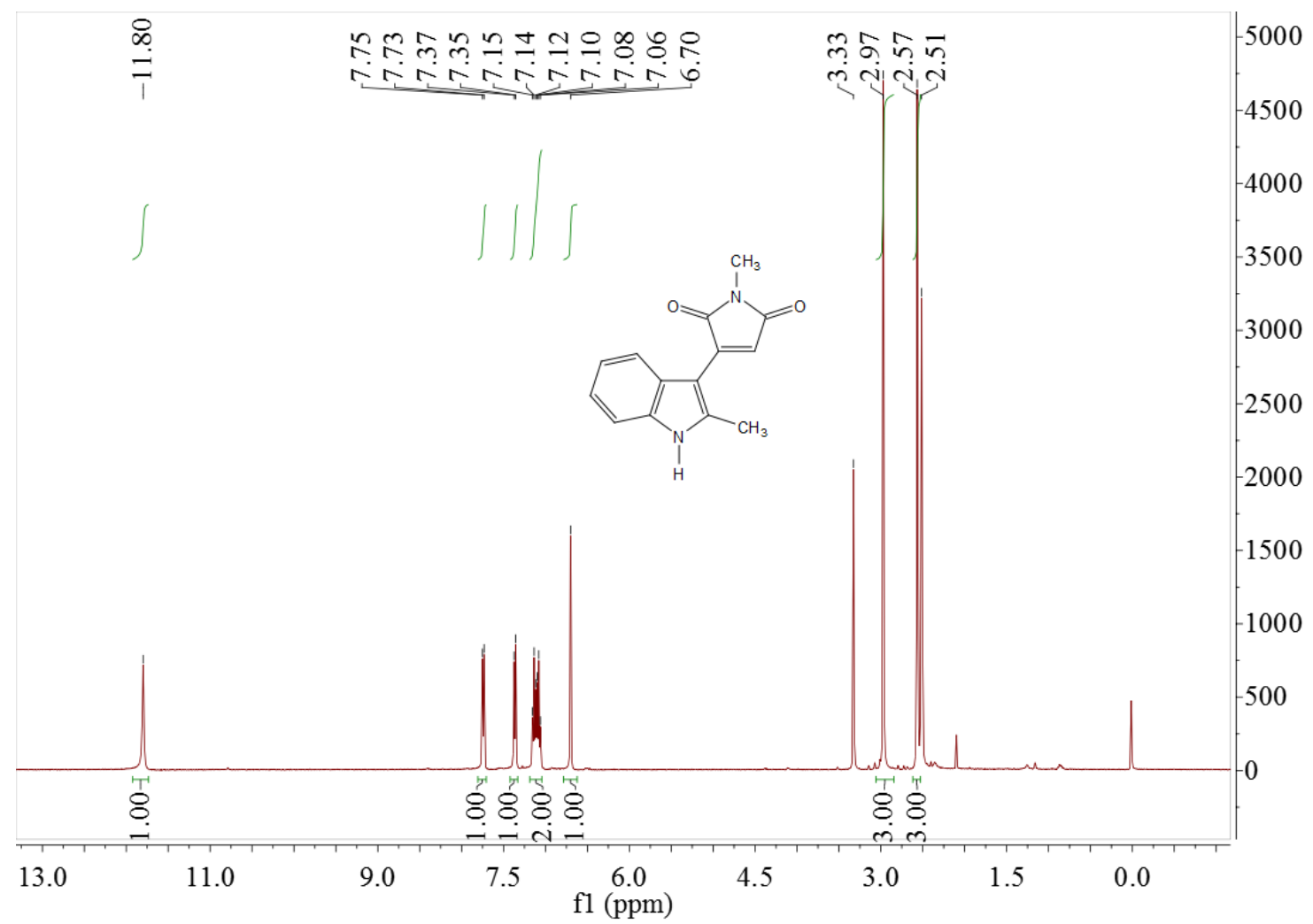

${ }^{1} \mathrm{H}$ NMR spectrum of 1-methyl-3-(2-methyl- $1 H$-indol-3-yl)- $1 H$-pyrrole-2,5-dione (4c)

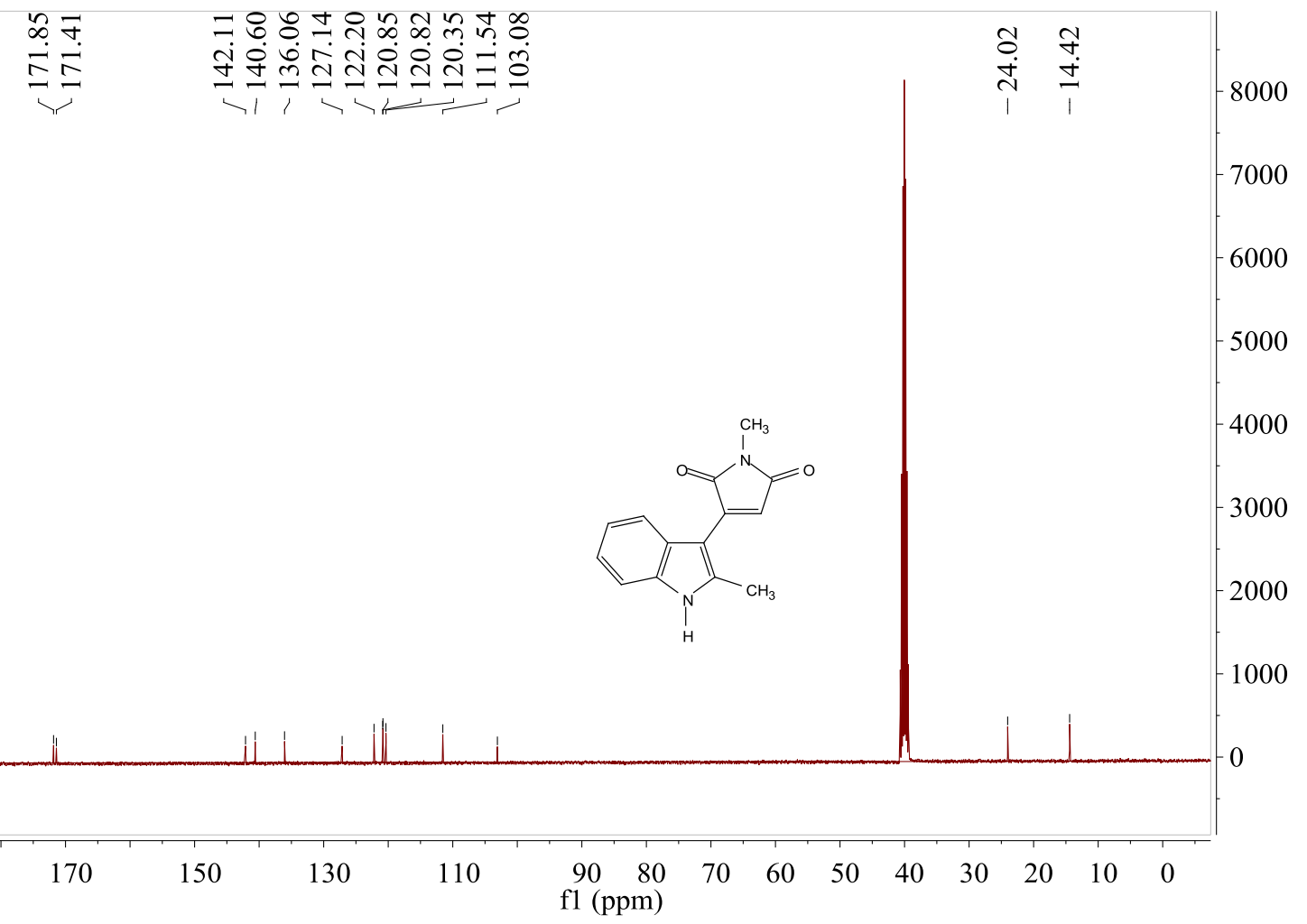

${ }^{13} \mathrm{C}$ NMR spectrum of 1-methyl-3-(2-methyl-1H-indol-3-yl)-1 $H$-pyrrole-2,5-dione (4c) 


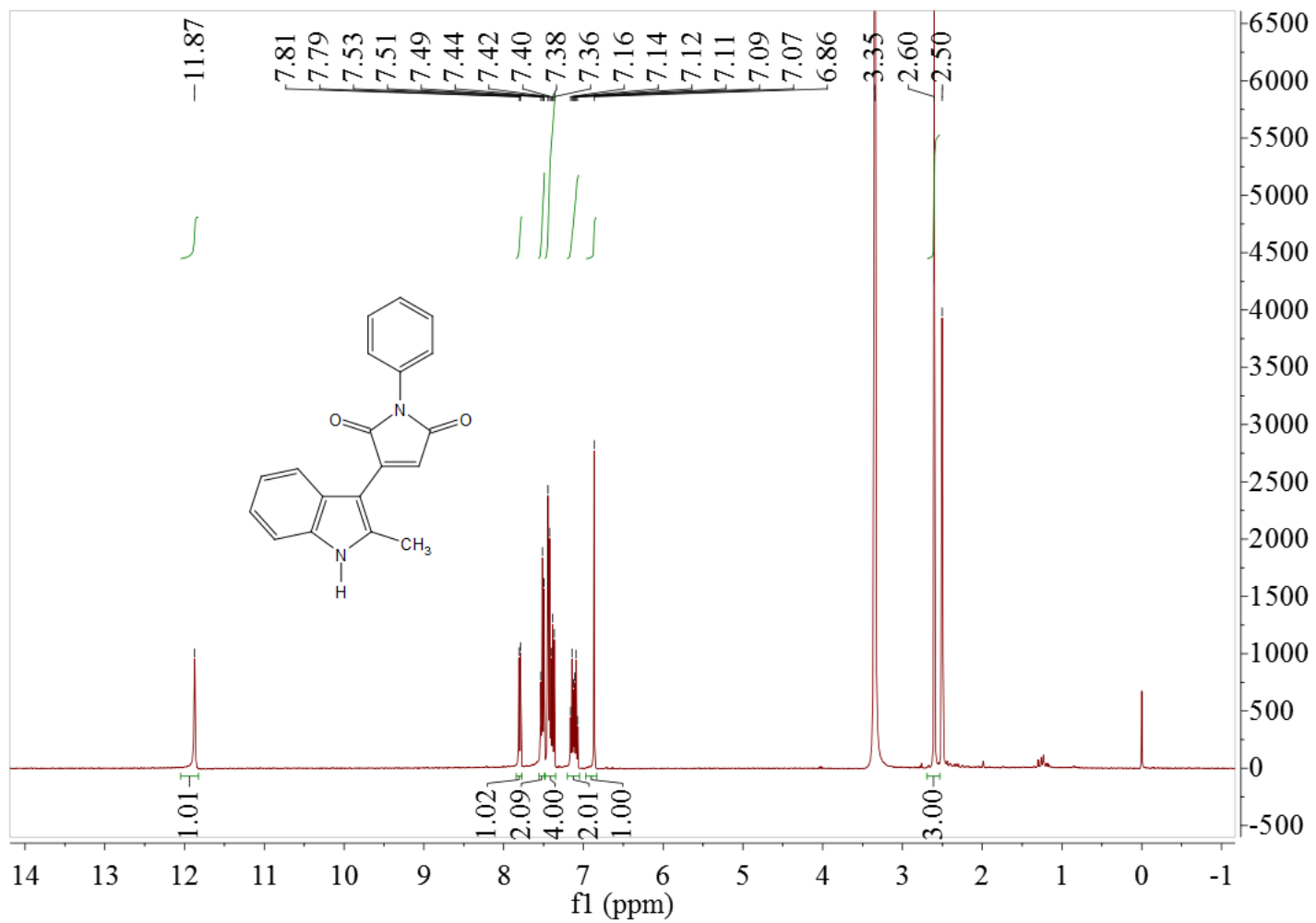

${ }^{1} \mathrm{H}$ NMR spectrum of 1-phenyl-3-(2-methyl- $1 H$-indol-3-yl)-1 $H$-pyrrole-2,5-dione (4d)
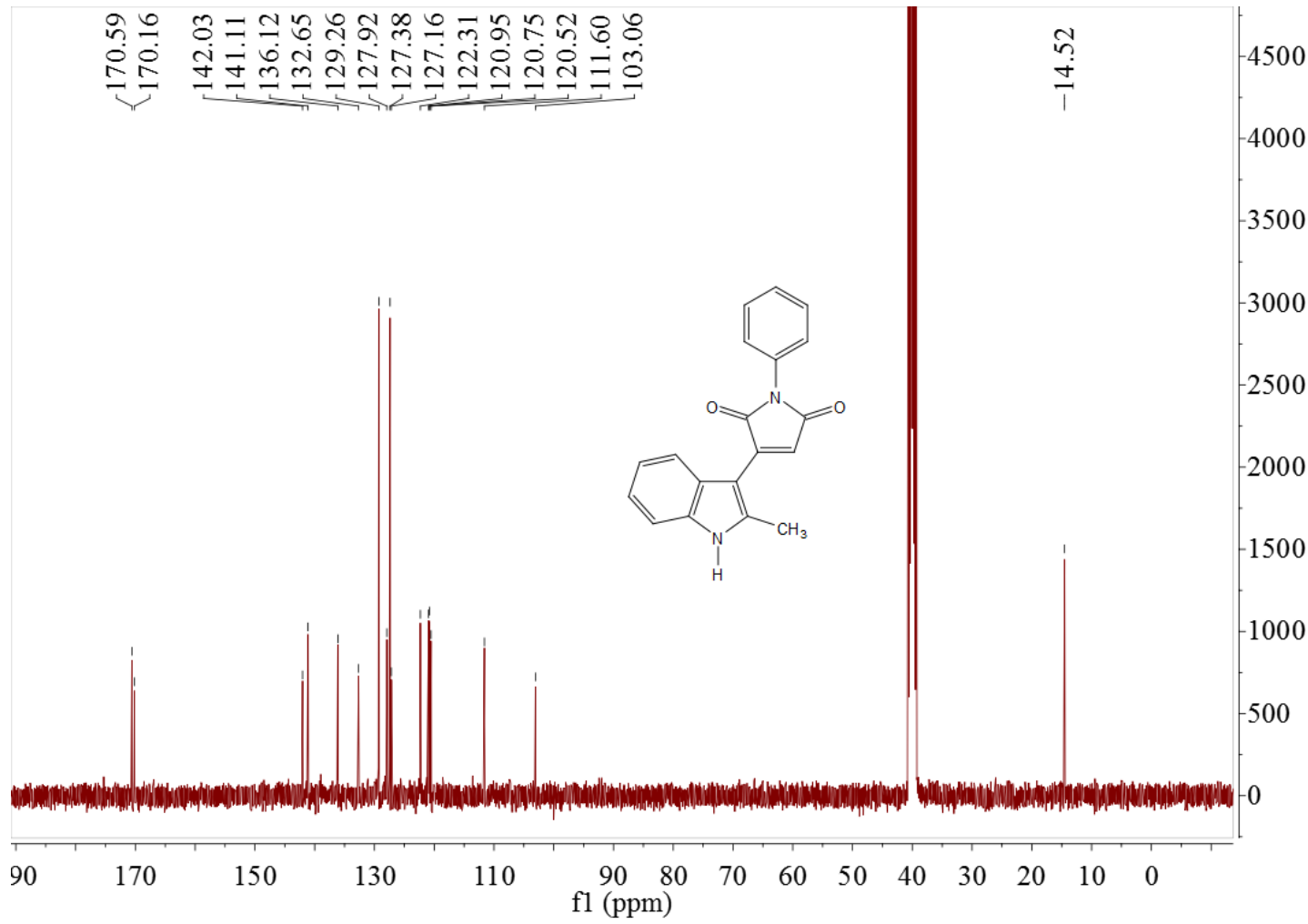

${ }^{13} \mathrm{C}$ NMR spectrum of 1-phenyl-3-(2-methyl- $1 H$-indol-3-yl)-1H -pyrrole-2,5-dione (4d) 


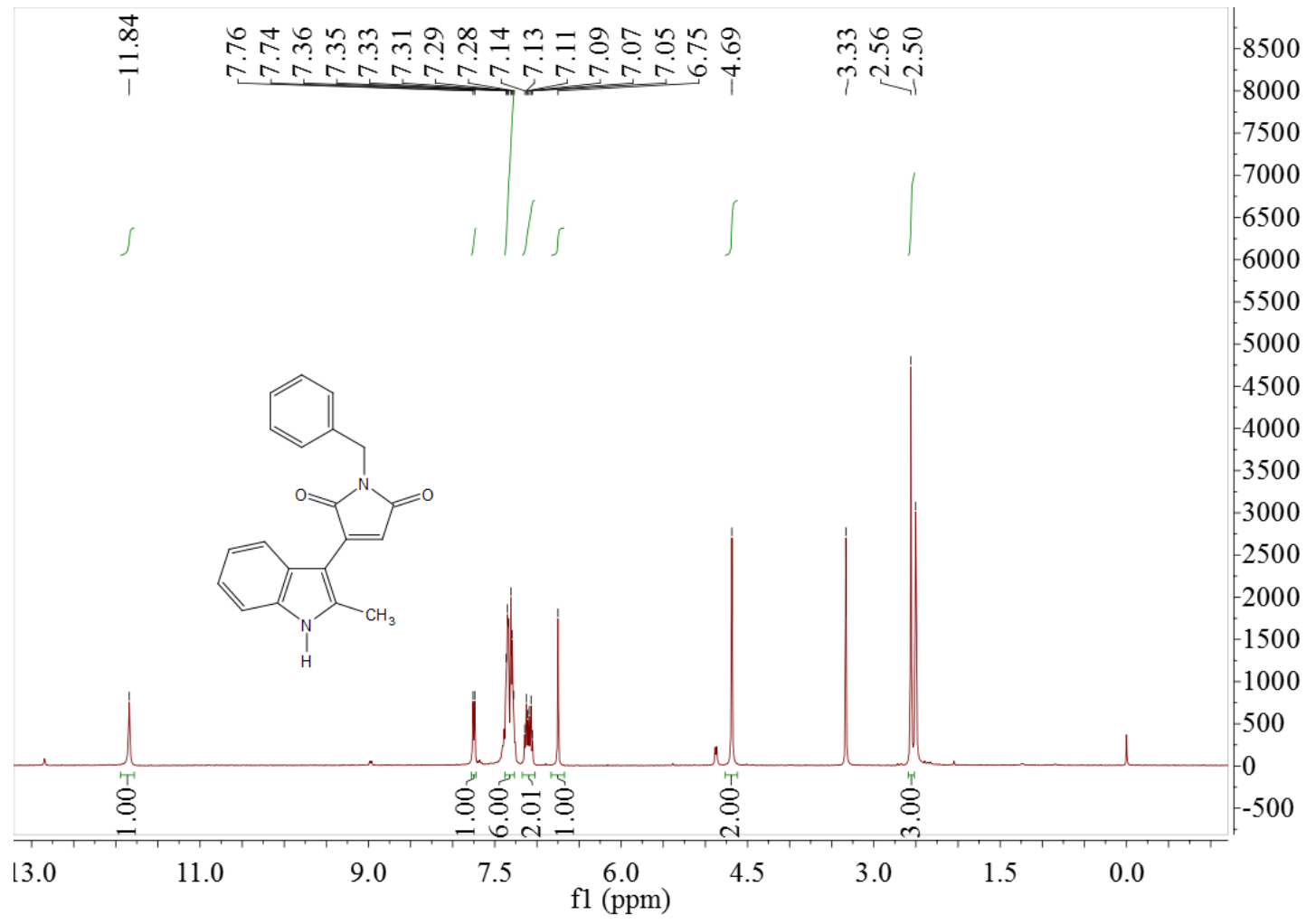

${ }^{1} \mathrm{H}$ NMR spectrum of 1-benzyl-3-(2-methyl- $1 H$-indol-3-yl)-1H -pyrrole-2,5-dione (4e)
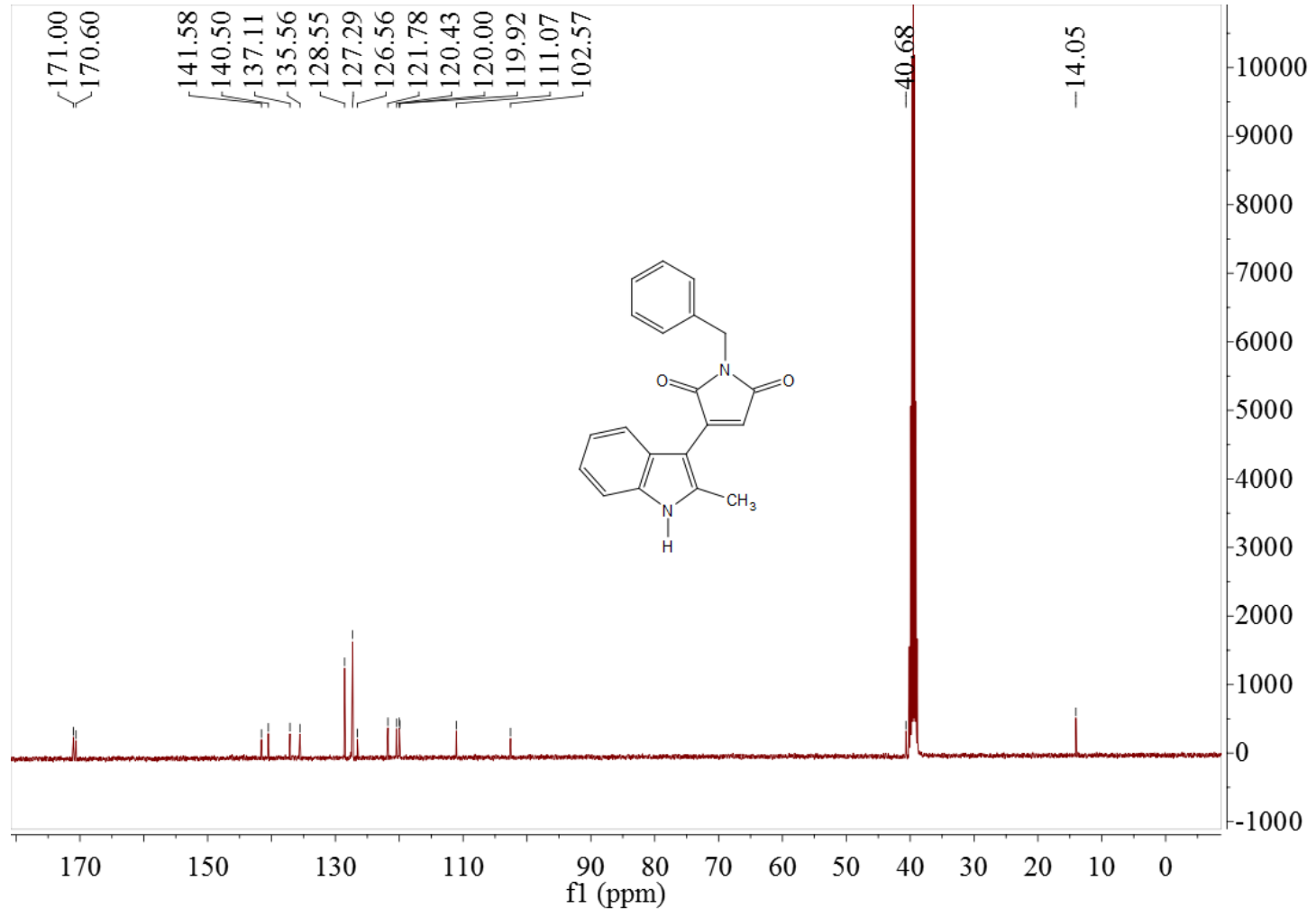

${ }^{13} \mathrm{C}$ NMR spectrum of 1-benzyl-3-(2-methyl- $1 H$-indol-3-yl)-1H -pyrrole-2,5-dione (4e) 


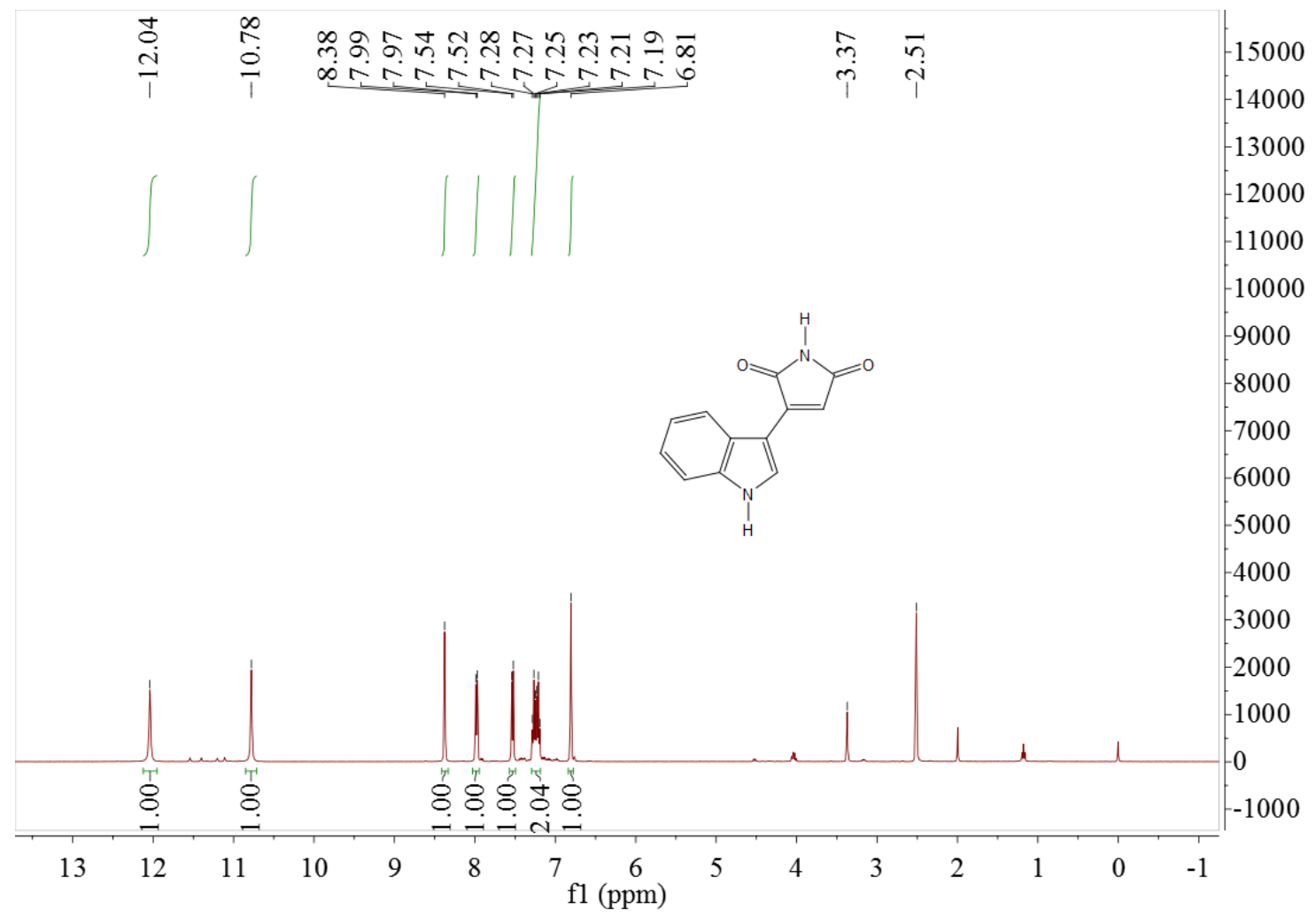

${ }^{1} \mathrm{H}$ NMR spectrum of 3-(1H-indol-3-yl)-1H-pyrrole-2,5-dione (4f)

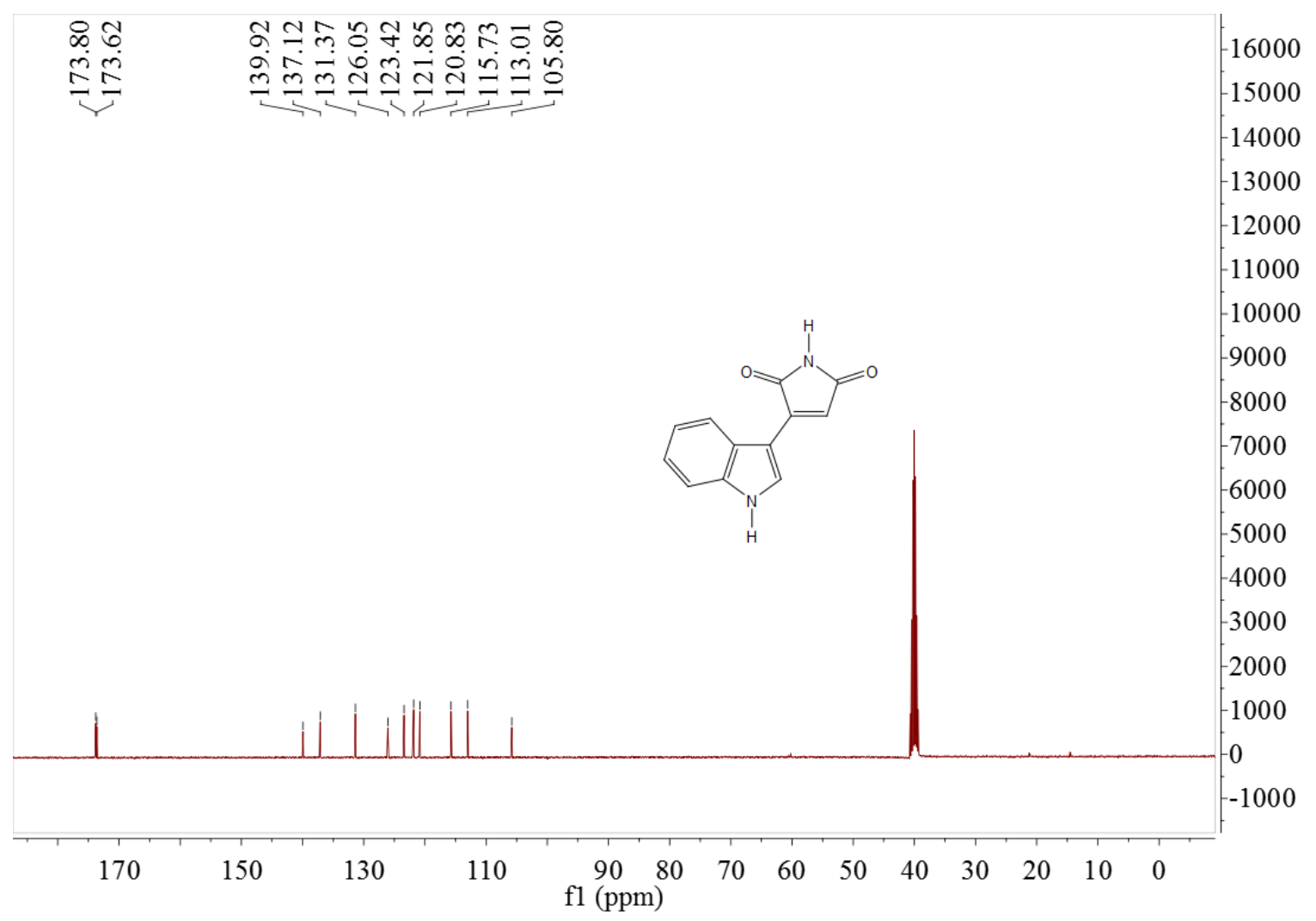

${ }^{13} \mathrm{C}$ NMR spectrum of 3-(1H-indol-3-yl)- $1 H$-pyrrole-2,5-dione (4f) 


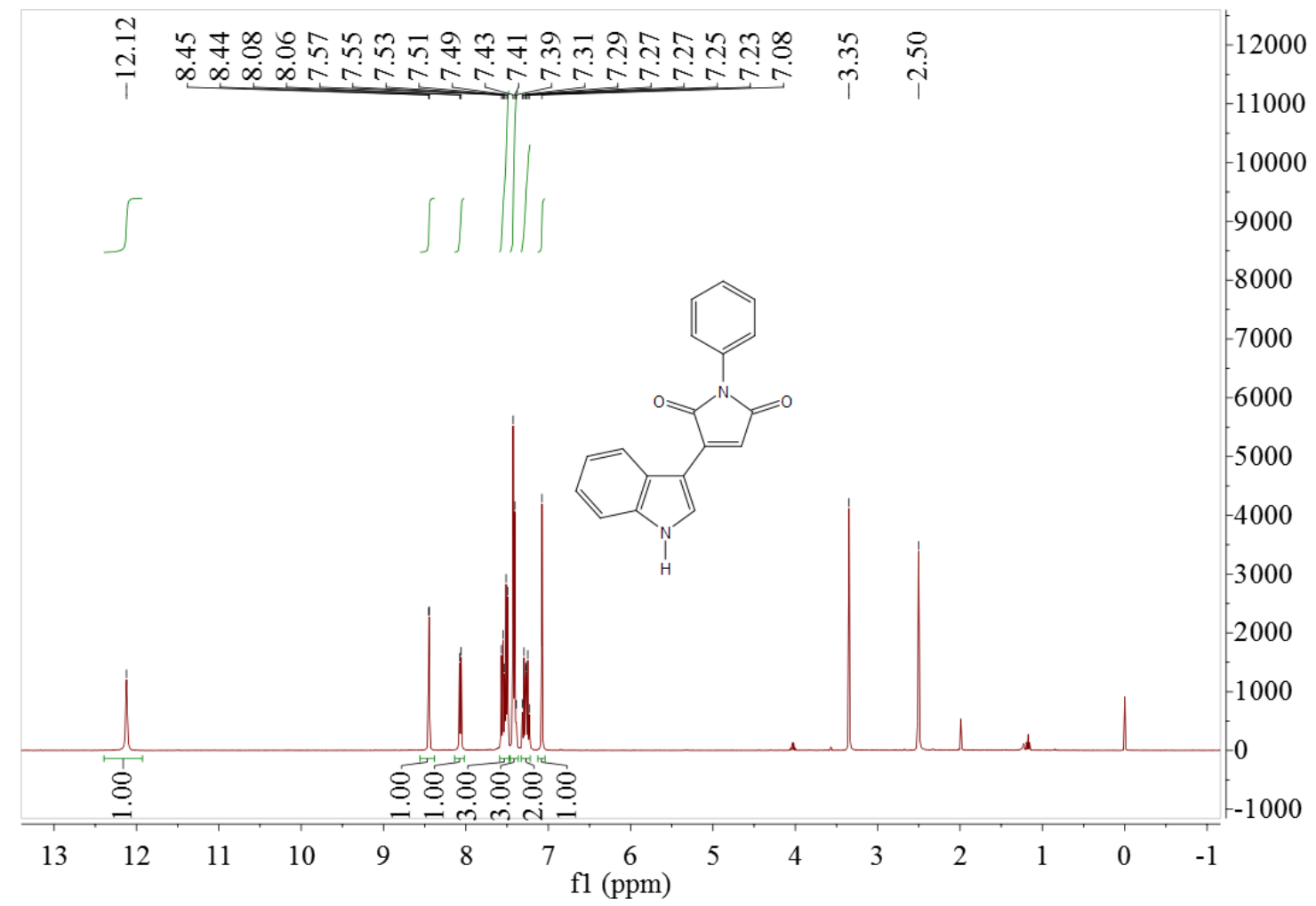

${ }^{1} \mathrm{H}$ NMR spectrum of 1-phenyl-3-(1H-indol-3-yl)-1H -pyrrole-2,5-dione (4g)
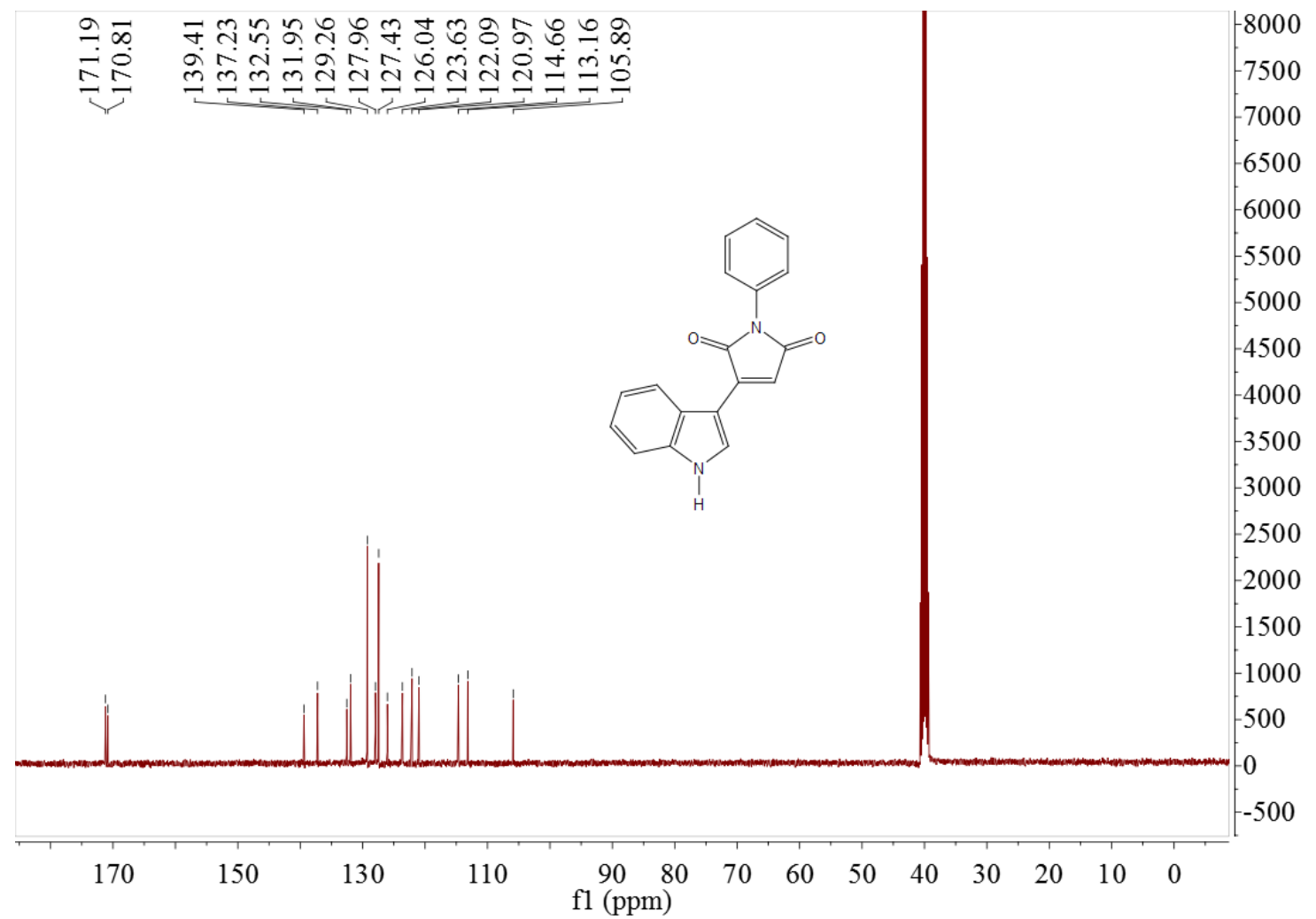

${ }^{13} \mathrm{C}$ NMR spectrum of 1-phenyl-3-(1H-indol-3-yl)- $1 H$-pyrrole-2,5-dione (4g) 


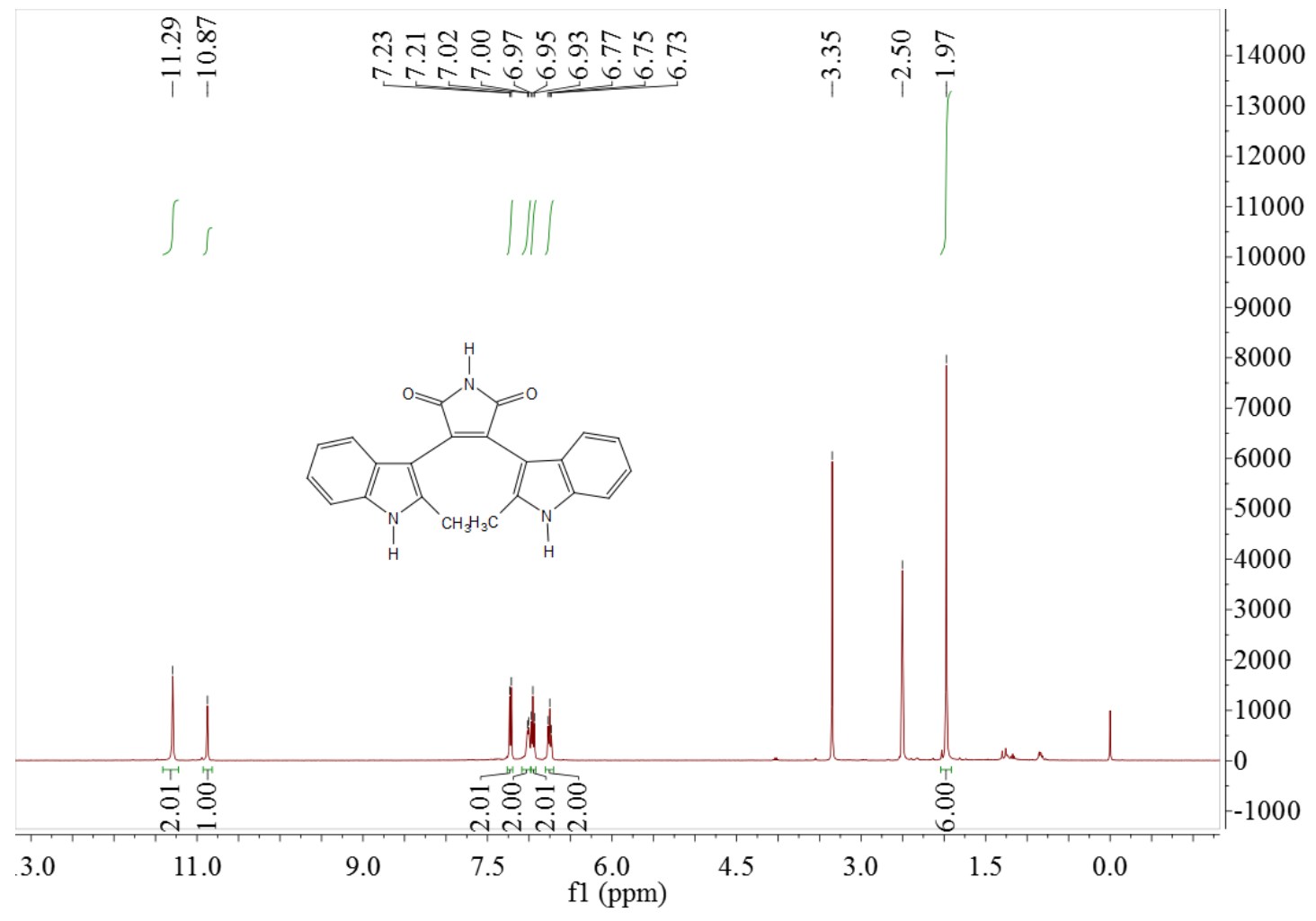

${ }^{1}$ H NMR spectrum of 3,4-di-(2-methyl- $1 H$-indol-3-yl)-1 $H$-pyrrole-2,5-dione (5a)

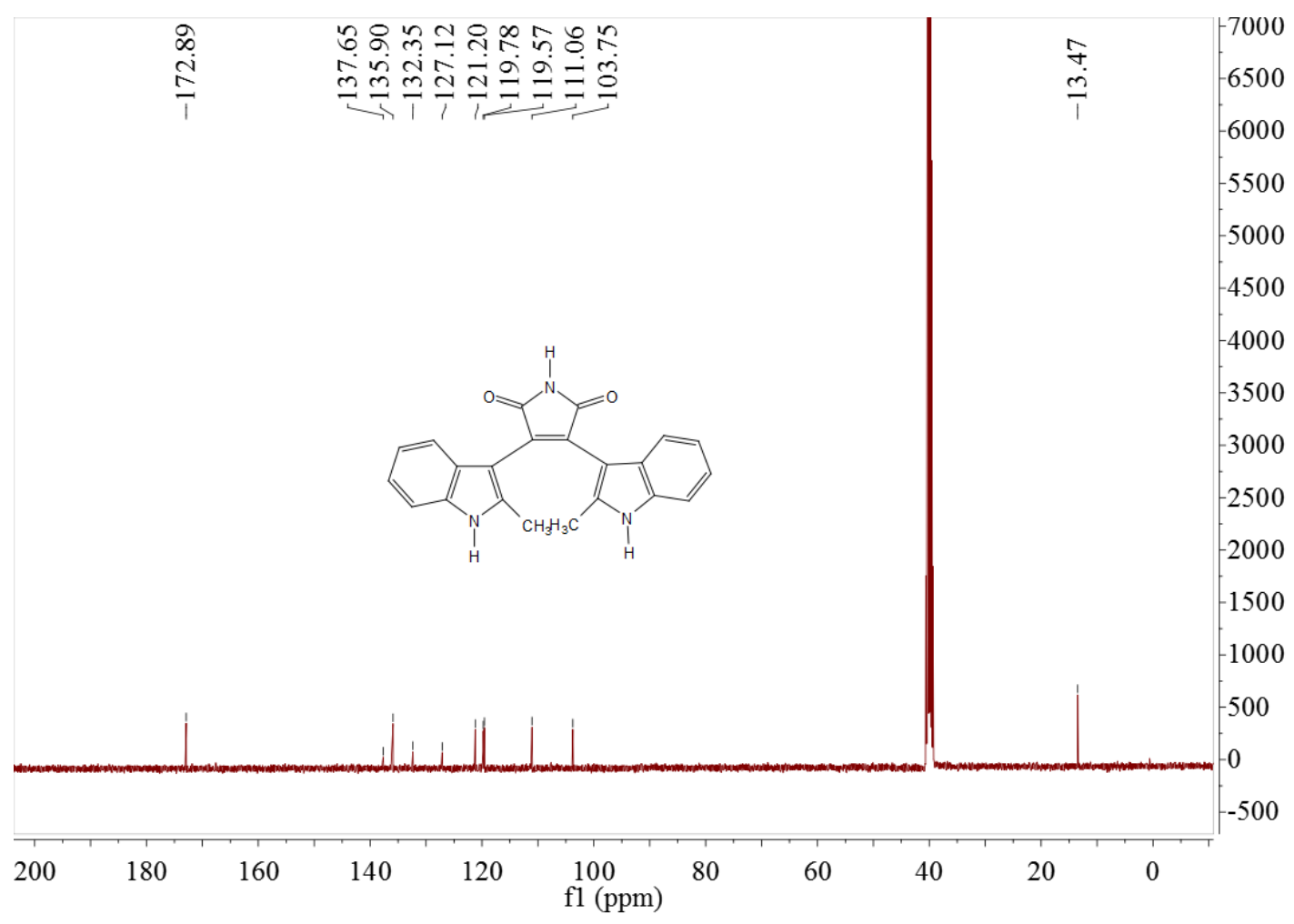

${ }^{13} \mathrm{C}$ NMR spectrum of 3,4-di-(2-methyl- $1 H$-indol-3-yl)- $1 H$-pyrrole-2,5-dione (5a) 


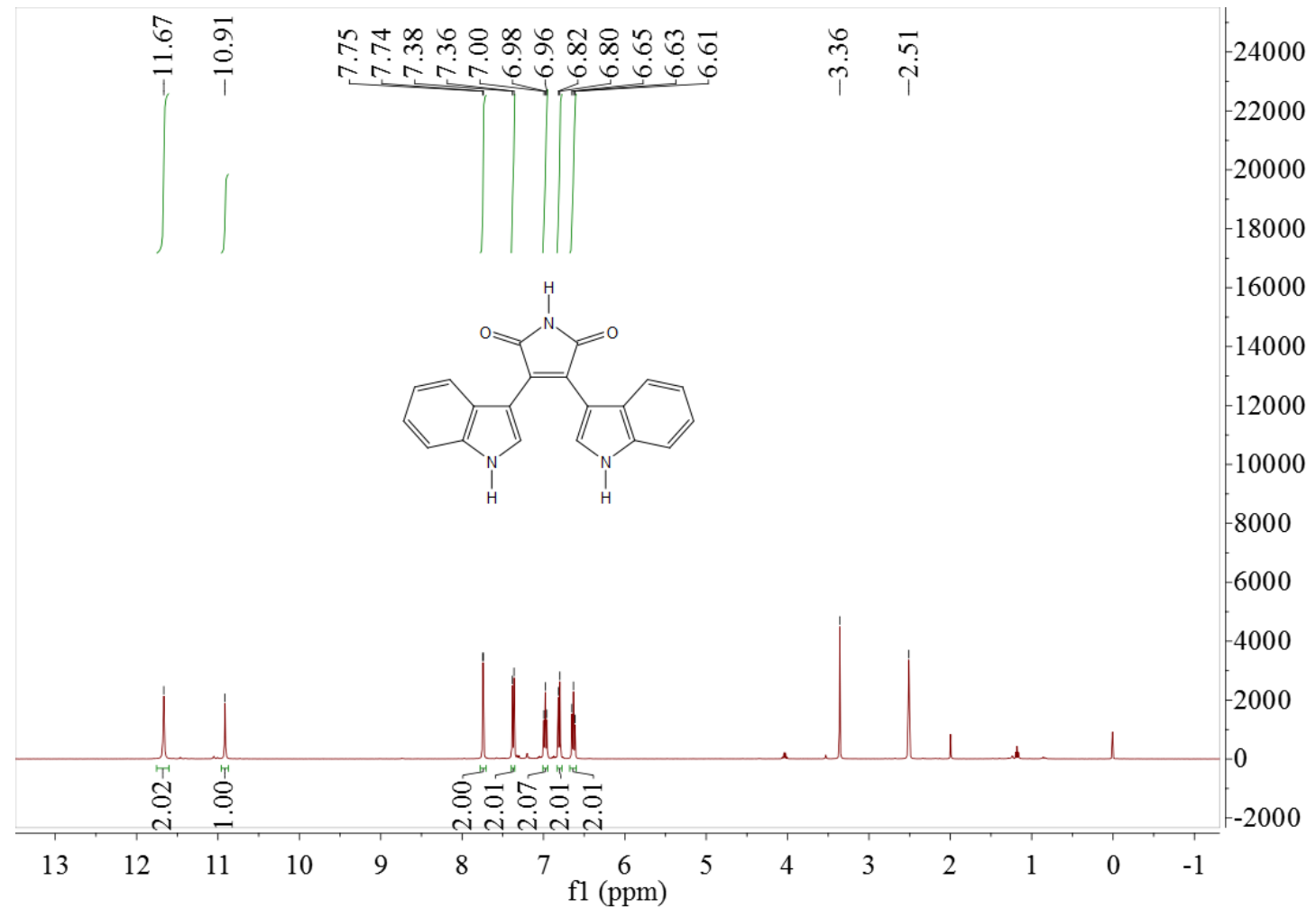

${ }^{1} \mathrm{H}$ NMR spectrum of 3,4-di-( $1 H$-indol-3-yl)- $1 H$-pyrrole-2,5-dione (5b)

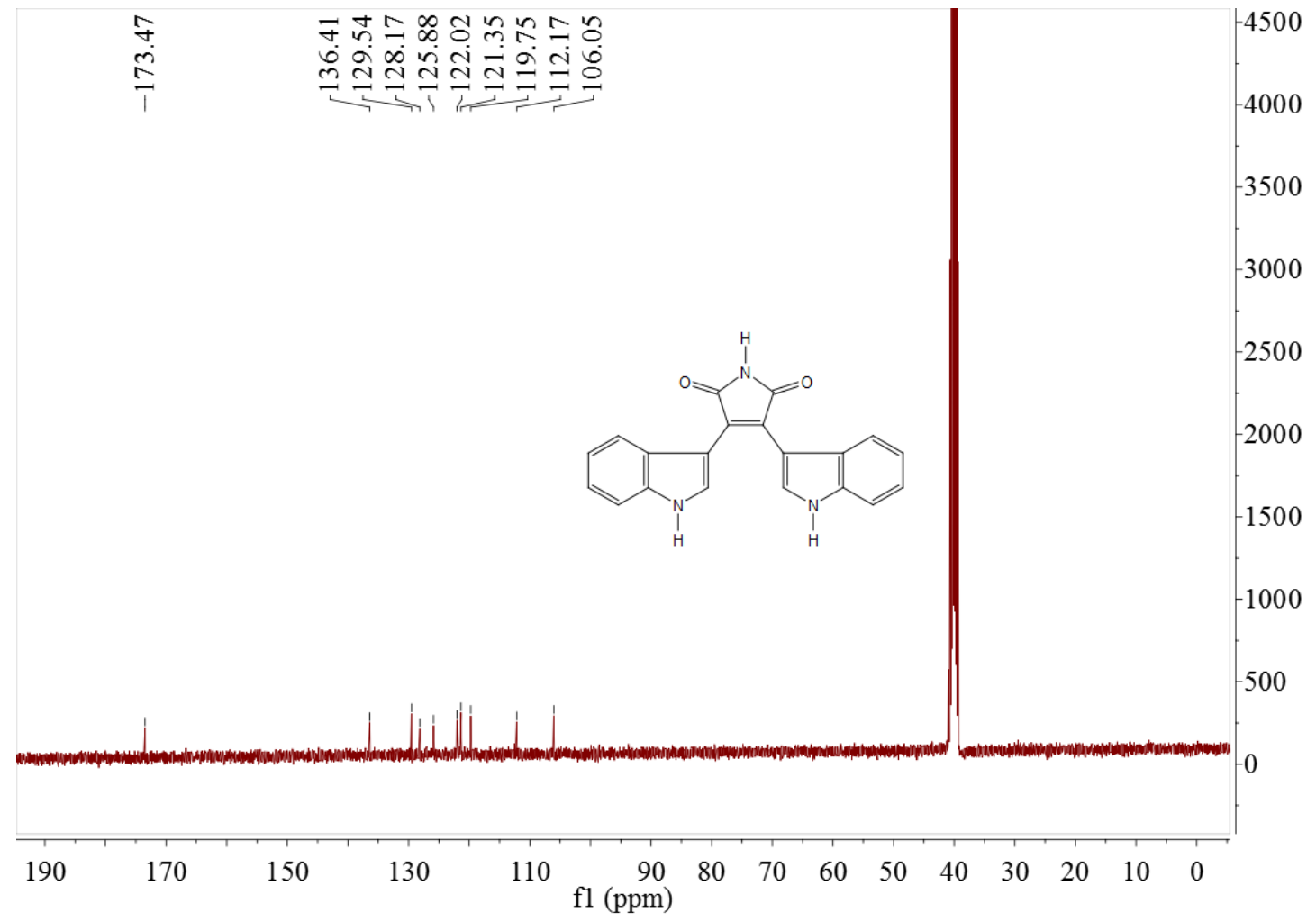

${ }^{13} \mathrm{C}$ NMR spectrum of 3,4-di-( $1 H$-indol-3-yl)- $1 H$-pyrrole-2,5-dione (5b) 


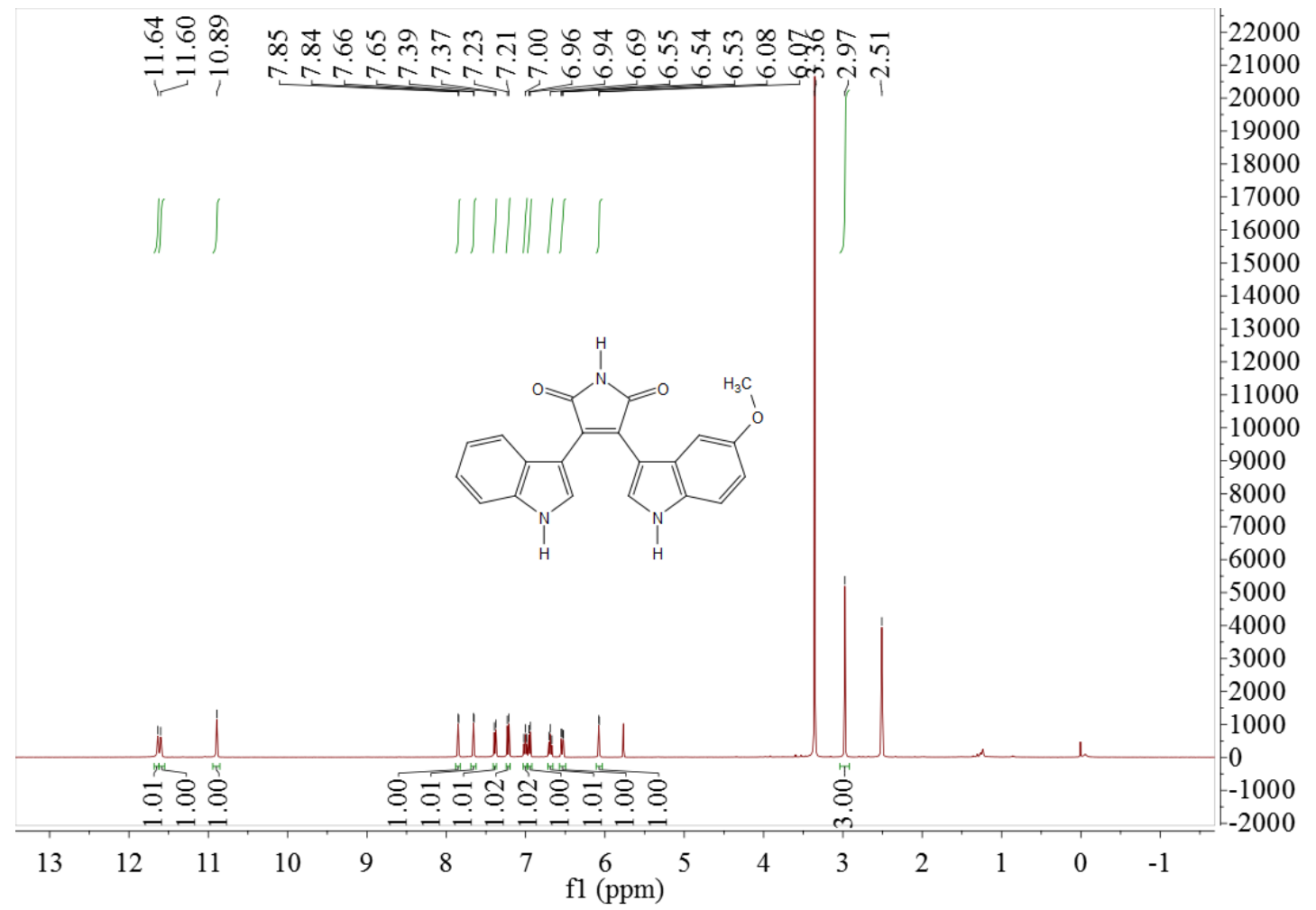

${ }^{1} \mathrm{H}$ NMR spectrum of $\quad 3$-( $1 H$-indol-3-yl)-4-(5-methoxy- $1 H$-indol-3-yl)- $1 H$-pyrrole-2,5-dione (5c)
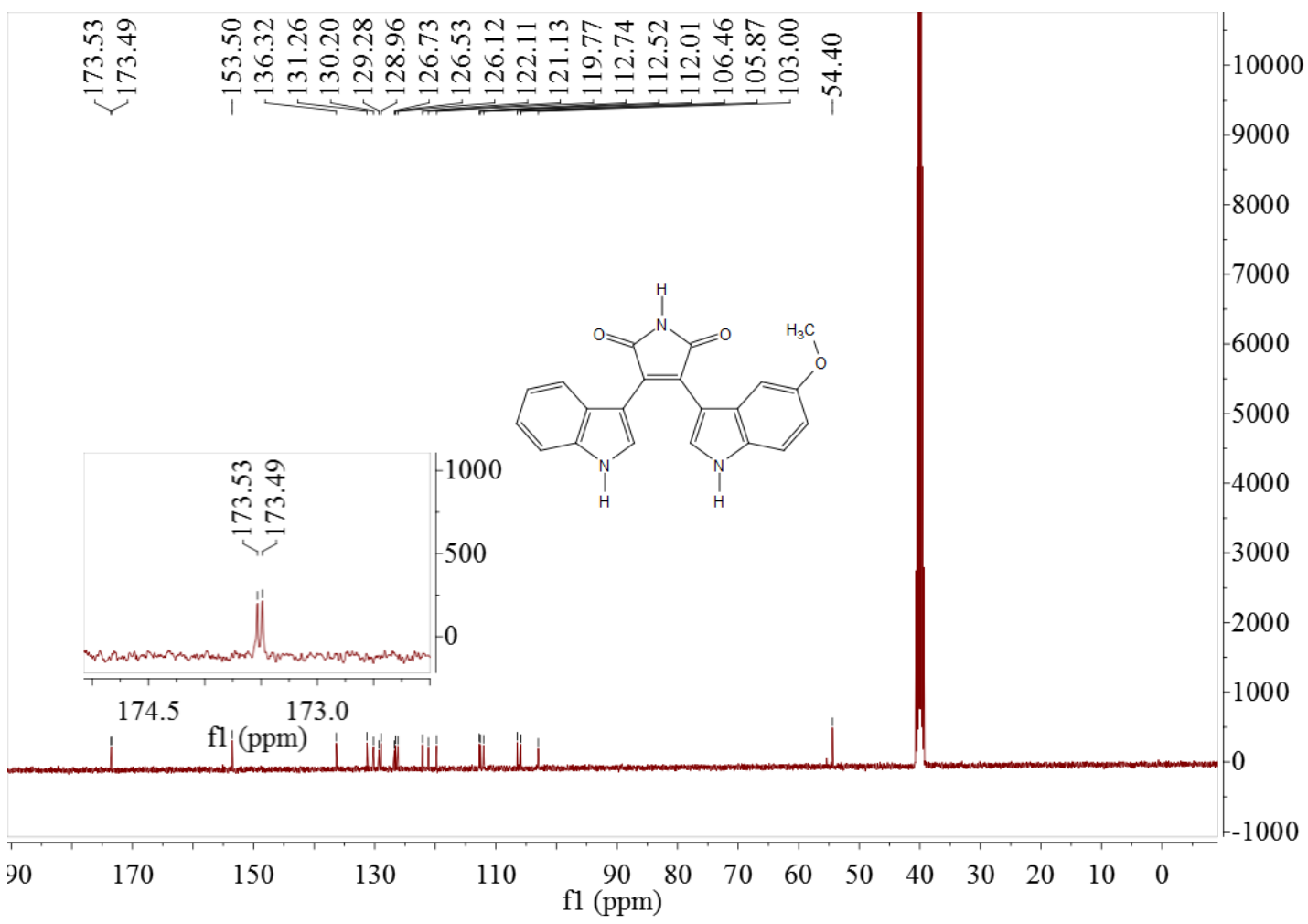

${ }^{13} \mathrm{C}$ NMR spectrum of 3-( $1 H$-indol-3-yl)-4-(5-methoxy- $1 H$-indol-3-yl)- $1 H$-pyrrole-2,5-dione (5c) 


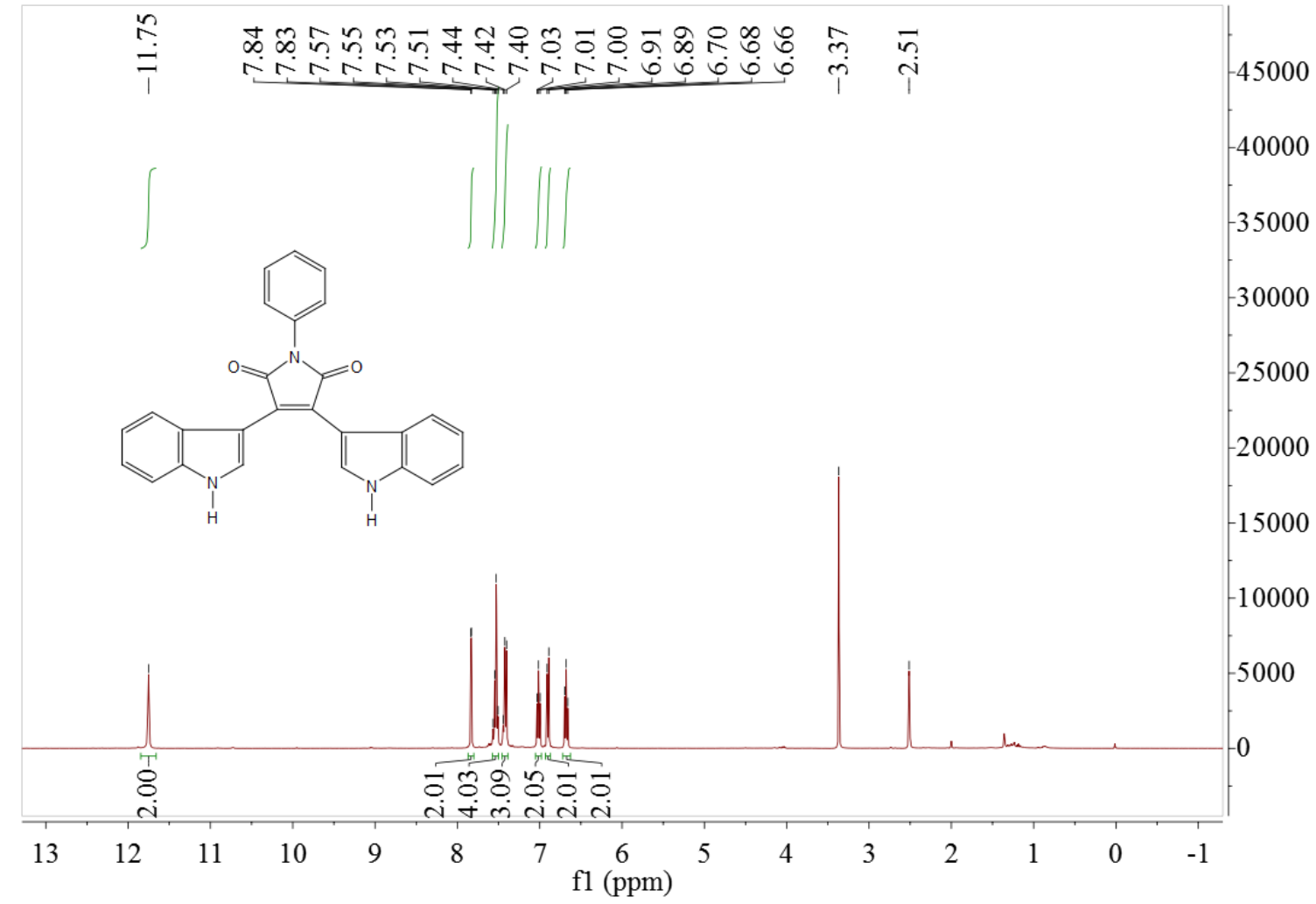

${ }^{1} \mathrm{H}$ NMR spectrum of 3,4-di-( $1 H$-indol-3-yl)- 1 -phenyl-1 $H$-pyrrole-2,5-dione (5d)

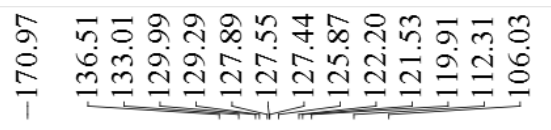

$$
\begin{aligned}
& \text { Lovuv } \\
& -26000 \\
& -24000 \\
& -22000 \\
& -20000 \\
& -18000 \\
& -16000 \\
& -14000 \\
& -12000 \\
& -10000 \\
& -8000 \\
& -6000 \\
& -4000 \\
& -2000 \\
& -0 \\
& -2000
\end{aligned}
$$

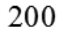

170

140

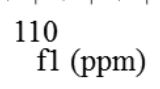

$80 \quad 60 \quad 40 \quad 20 \quad 0$

${ }^{13} \mathrm{C}$ NMR spectrum of 3,4-di-( $1 H$-indol-3-yl)-1-phenyl-1 $H$-pyrrole-2,5-dione (5d) 


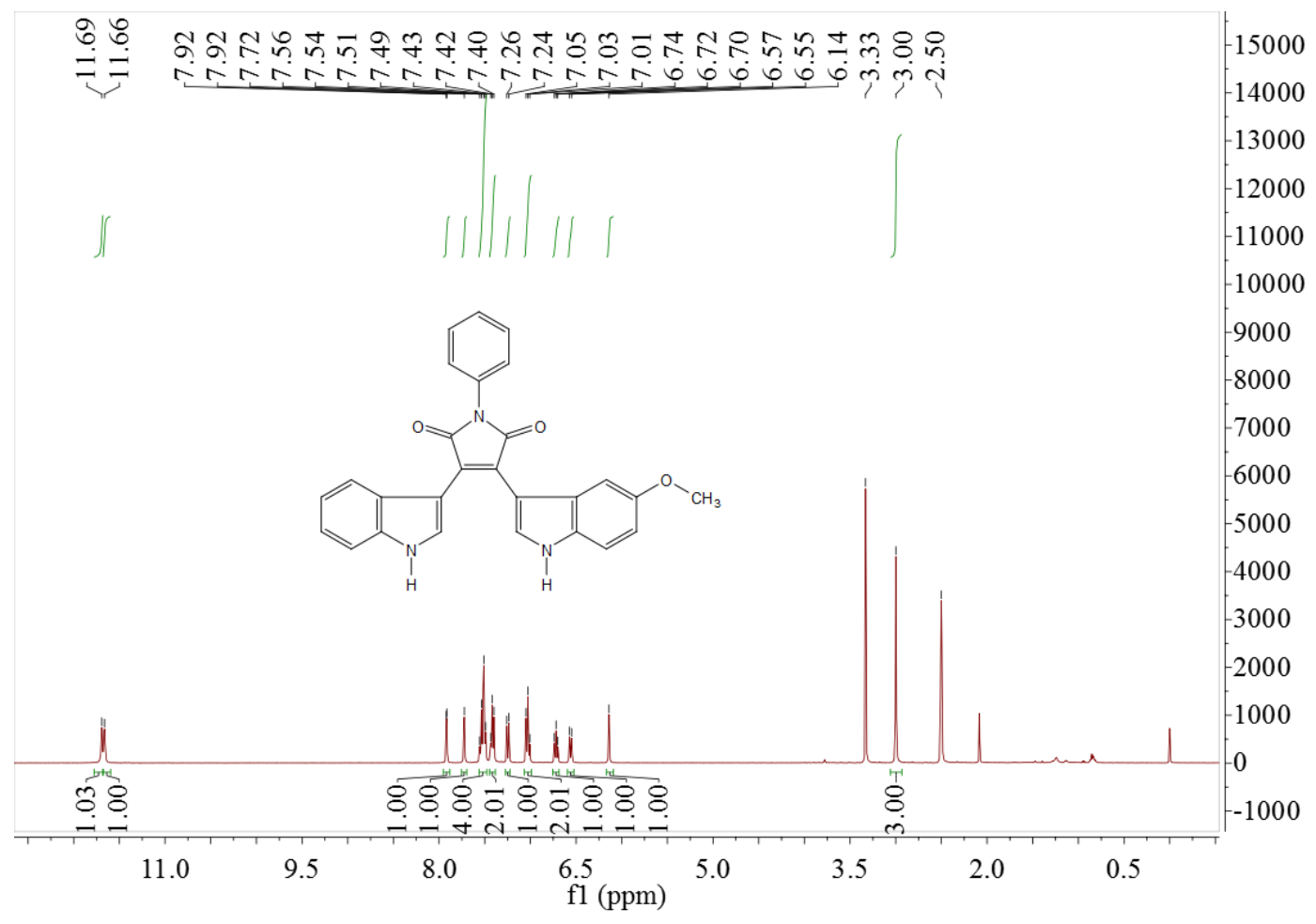

${ }^{1} \mathrm{H}$ NMR spectrum of 3-(1H-indol-3-yl)-4-(5-methoxy- $1 H$-indol-3-yl)- $N$-phenyl-pyrrole-2,5-dione (5e)

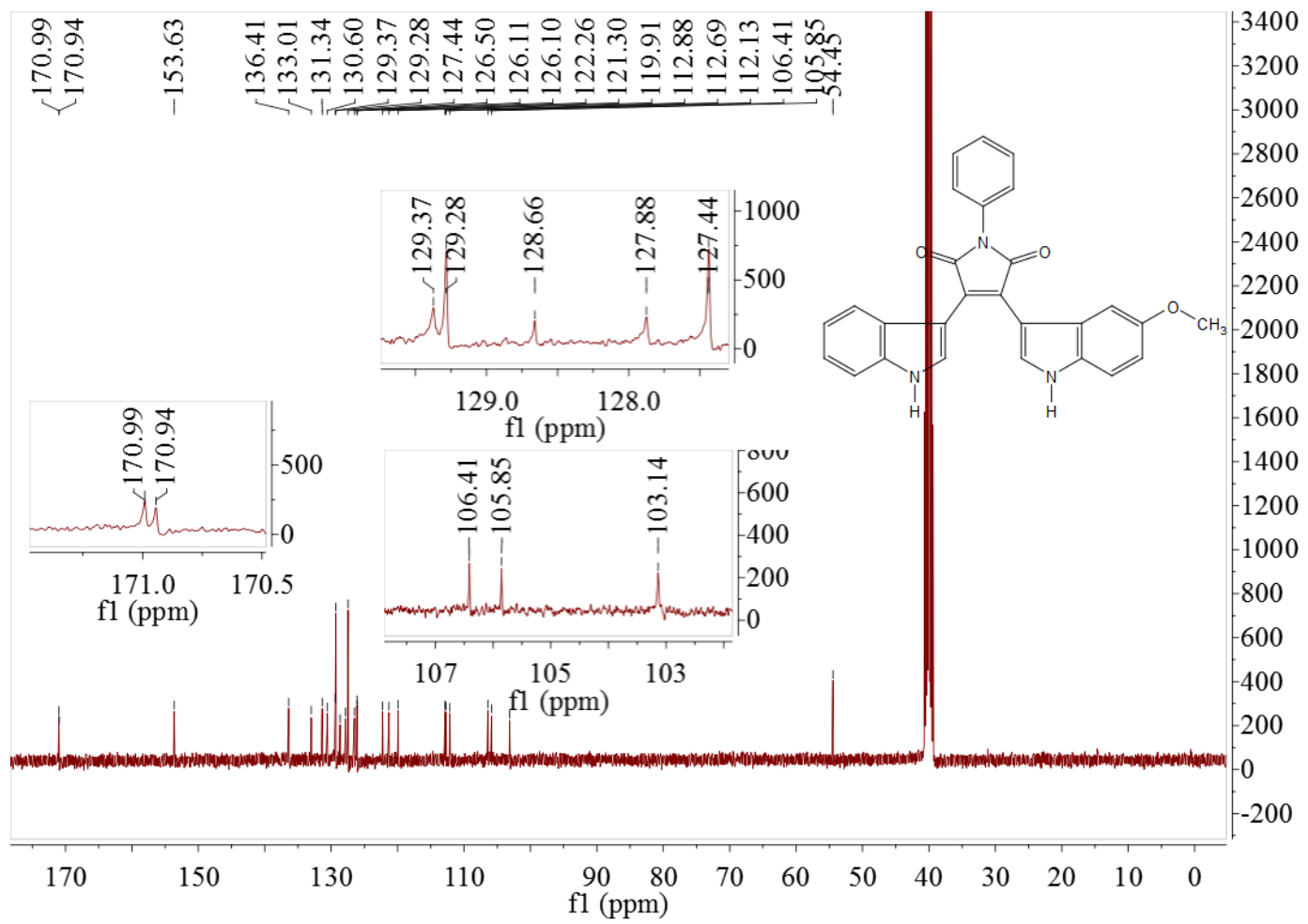

${ }^{13} \mathrm{C}$ NMR spectrum of 3-( $1 H$-indol-3-yl)-4-(5-methoxy- $1 H$-indol-3-yl)-1-phenyl-1 $H$-pyrrole-2,5-dione (5e) 


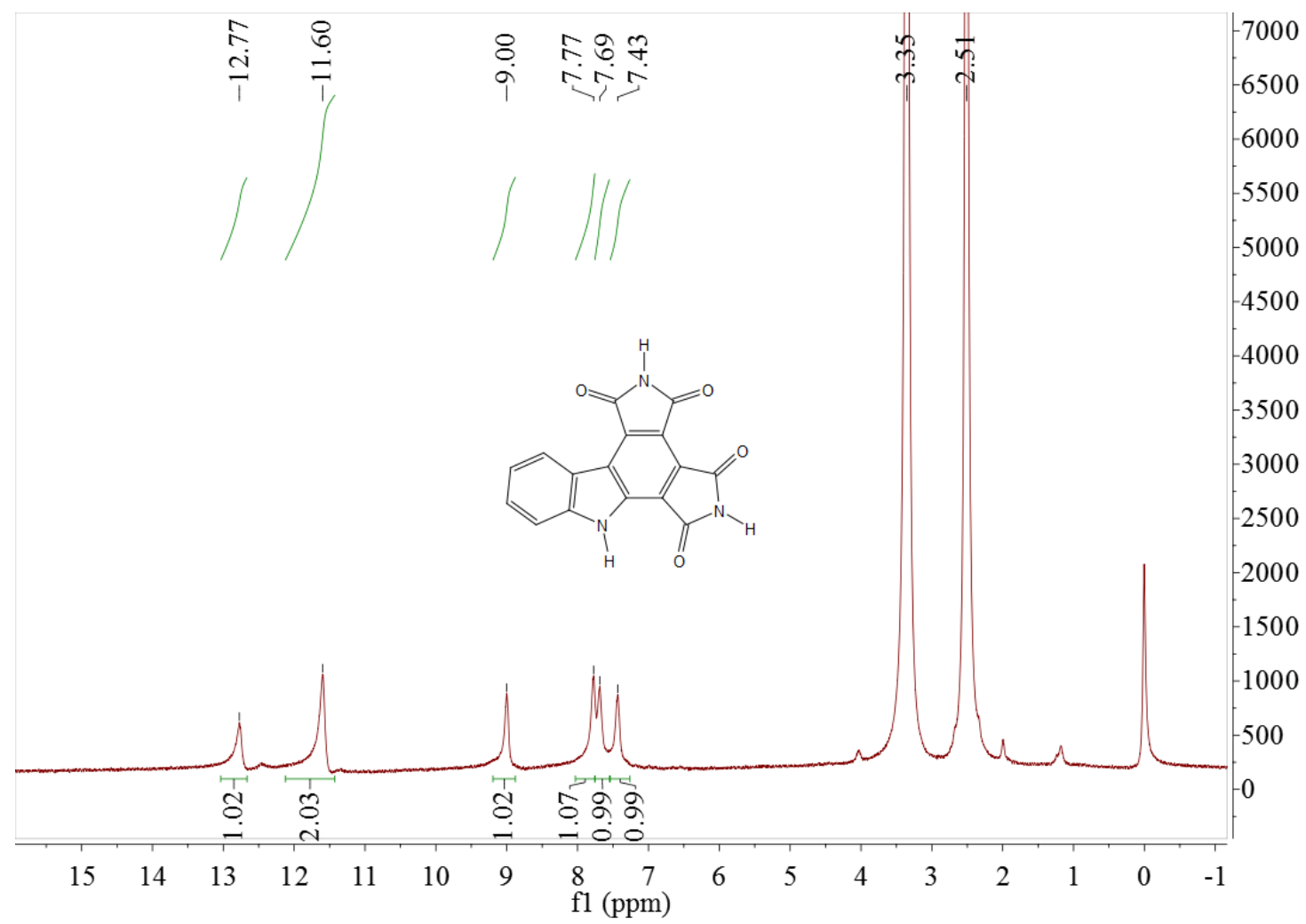

${ }^{1} \mathrm{H}$ NMR spectrum of $1 H$-dipyrrolo[3,4- $a: 3$ ',4'-c]carbazole-1,3,4,6(2H,5H,7H)-tetrone (7a)

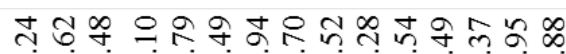

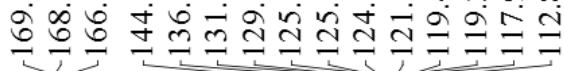

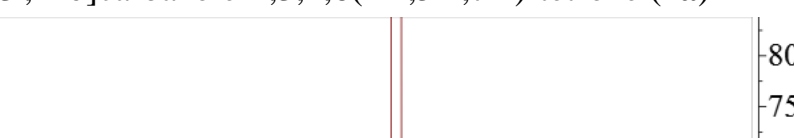

8000

7500

7000

$-6500$

6000

5500

5000

4500

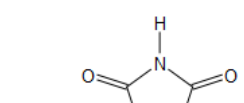

$-4000$

$-3500$

3000

2500

2000

$-1500$

1000

500

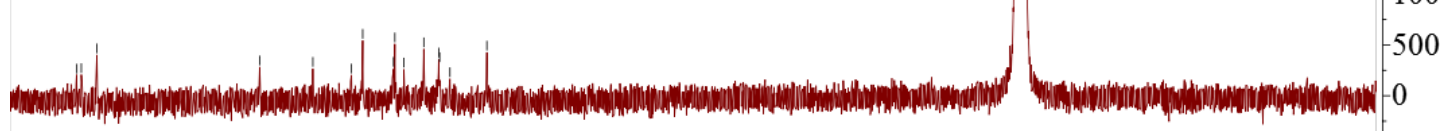

$-500$

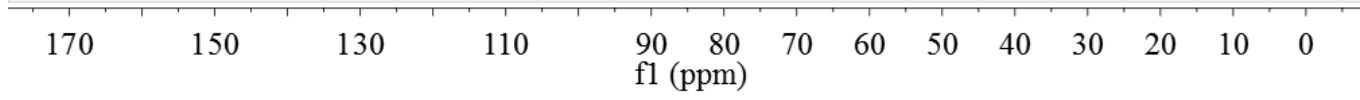

${ }^{13} \mathrm{C}$ NMR spectrum of $1 H$-dipyrrolo[3,4-a:3',4'-c] carbazole-1,3,4,6(2H,5H,7H)-tetrone (7a) 


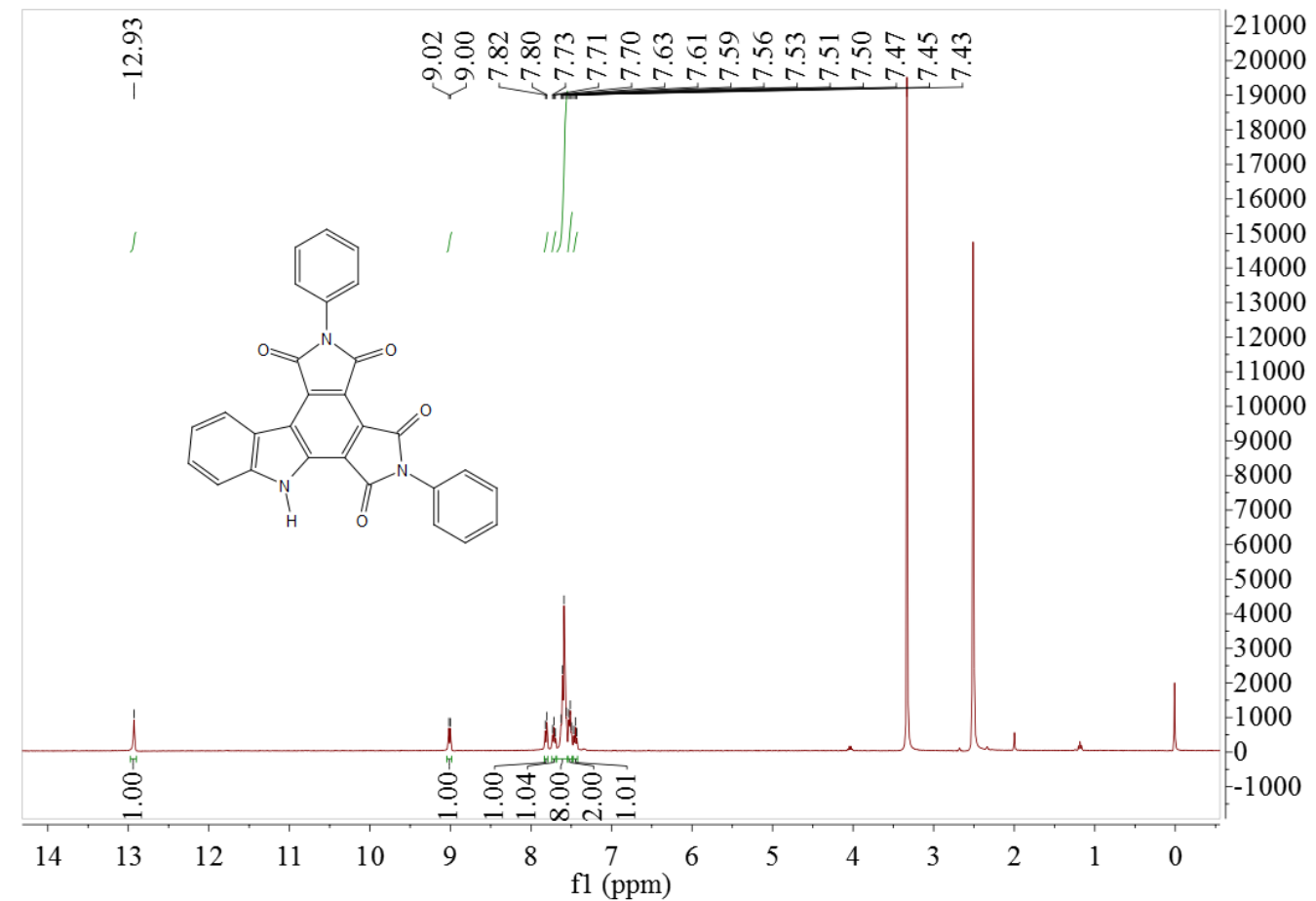

${ }^{1} \mathrm{H}$ NMR spectrum of 2,5-diphenyl-1H-dipyrrolo[3,4-a:3',4'-c]carbazole-1,3,4,6(2H,5H,7H)-tetrone (7b)

윻ำ

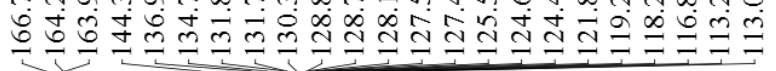

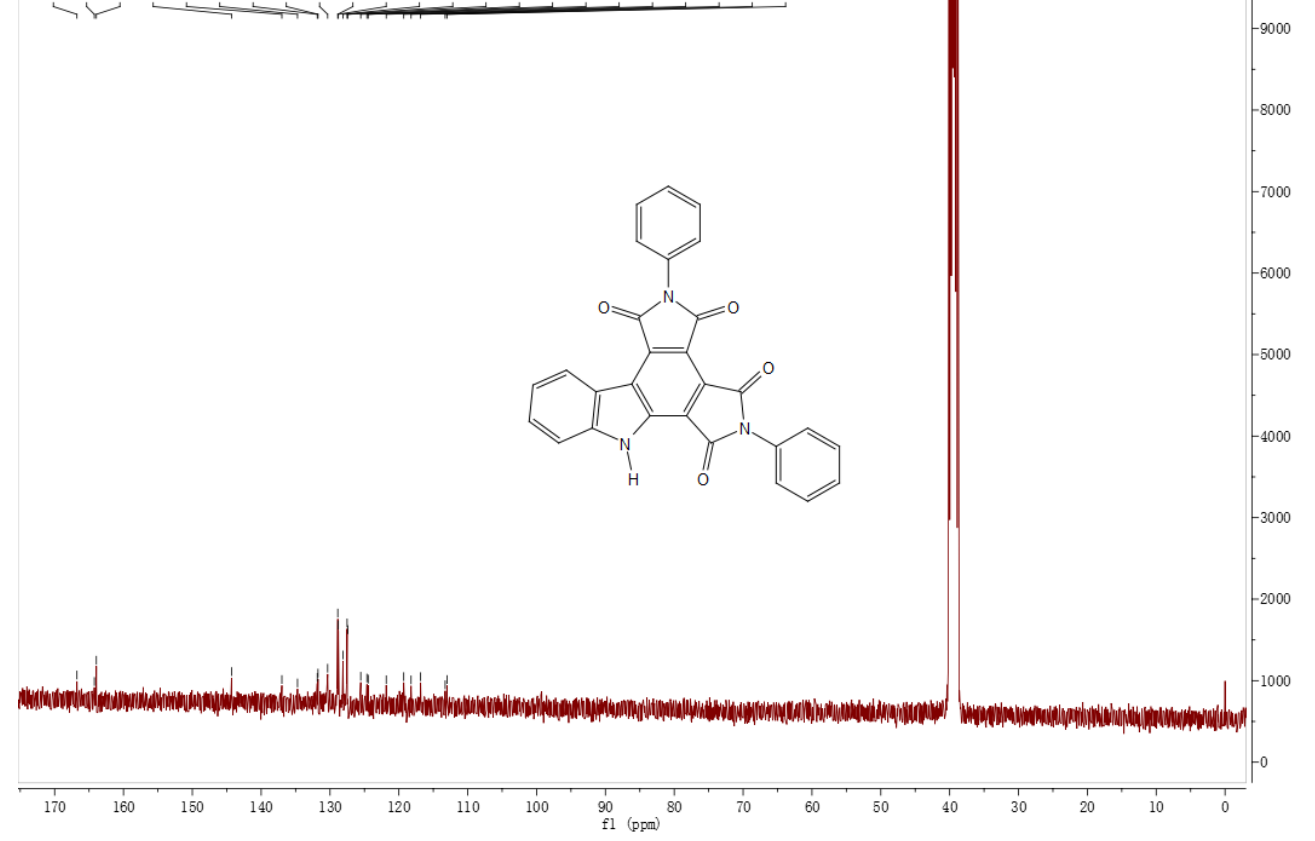

${ }^{13} \mathrm{C}$ NMR spectrum of 2,5-diphenyl-1H-dipyrrolo[3,4- $a: 3$ ',4'-c]carbazole-1,3,4,6(2H,5H,7H)-tetrone (7b) 


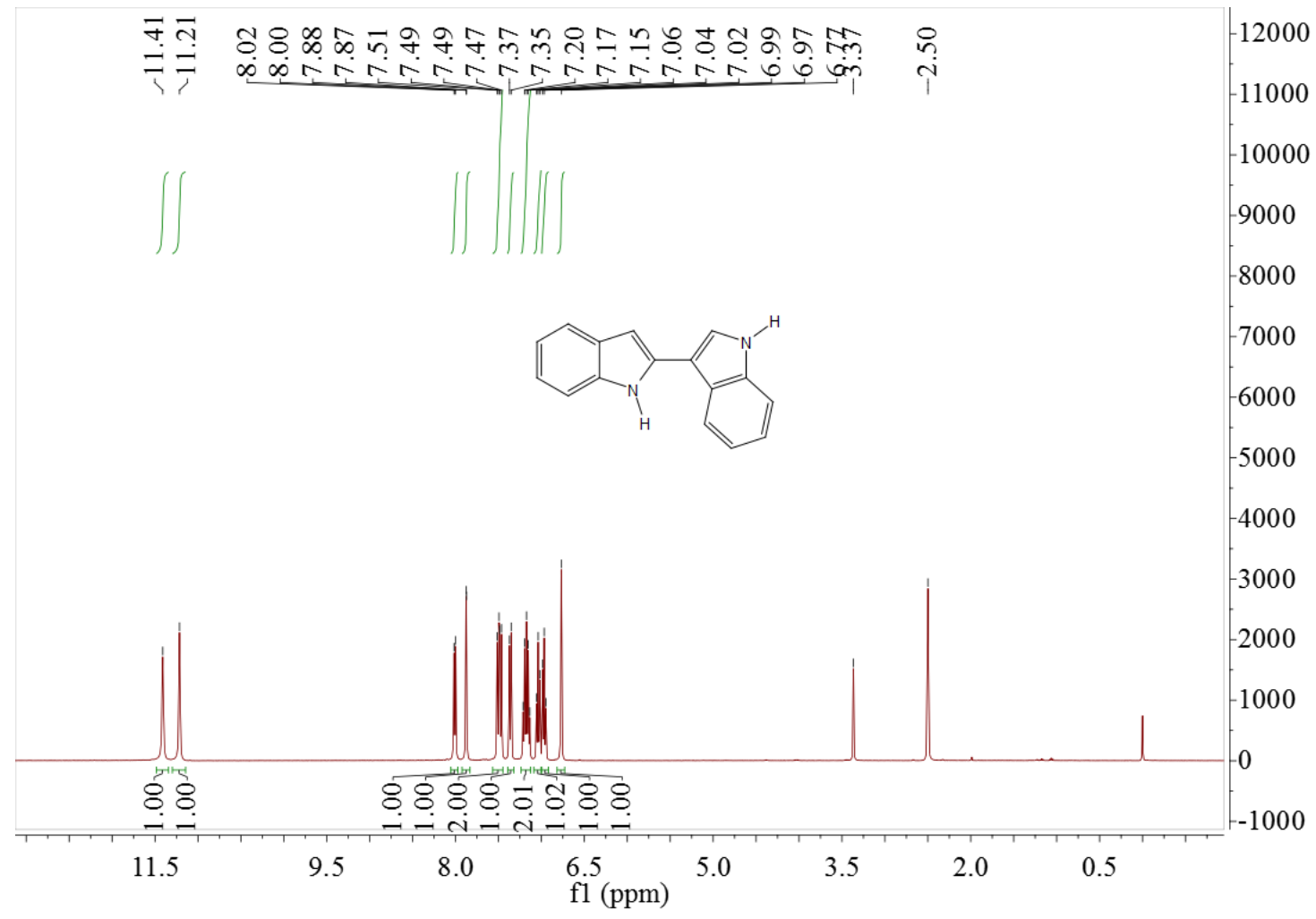

${ }^{1} \mathrm{H}$ NMR spectrum of 2,3'-bi- $1 H$-indole (8a)

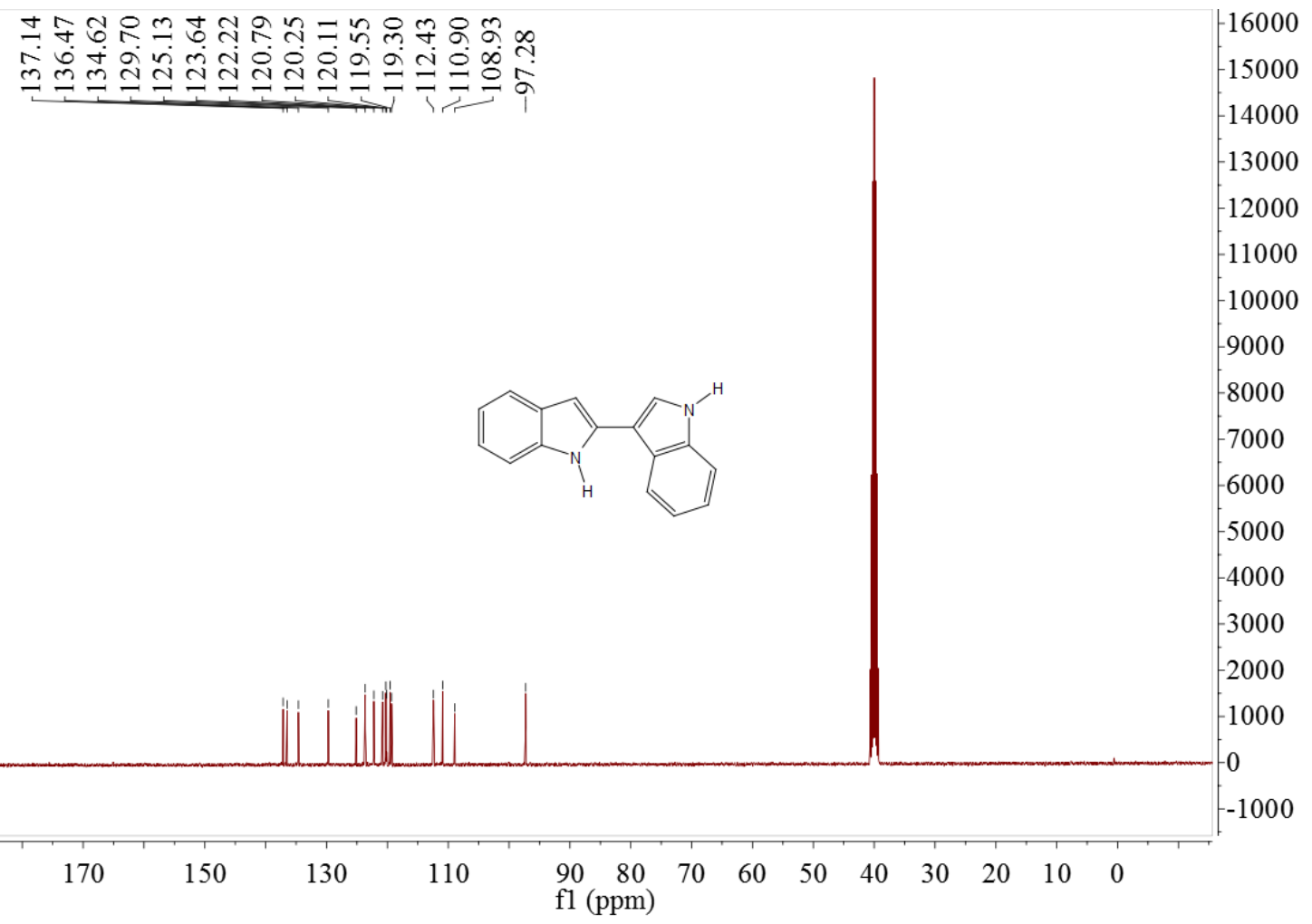

${ }^{13} \mathrm{C}$ NMR spectrum of 2,3'-bi-1H-indole (8a) 


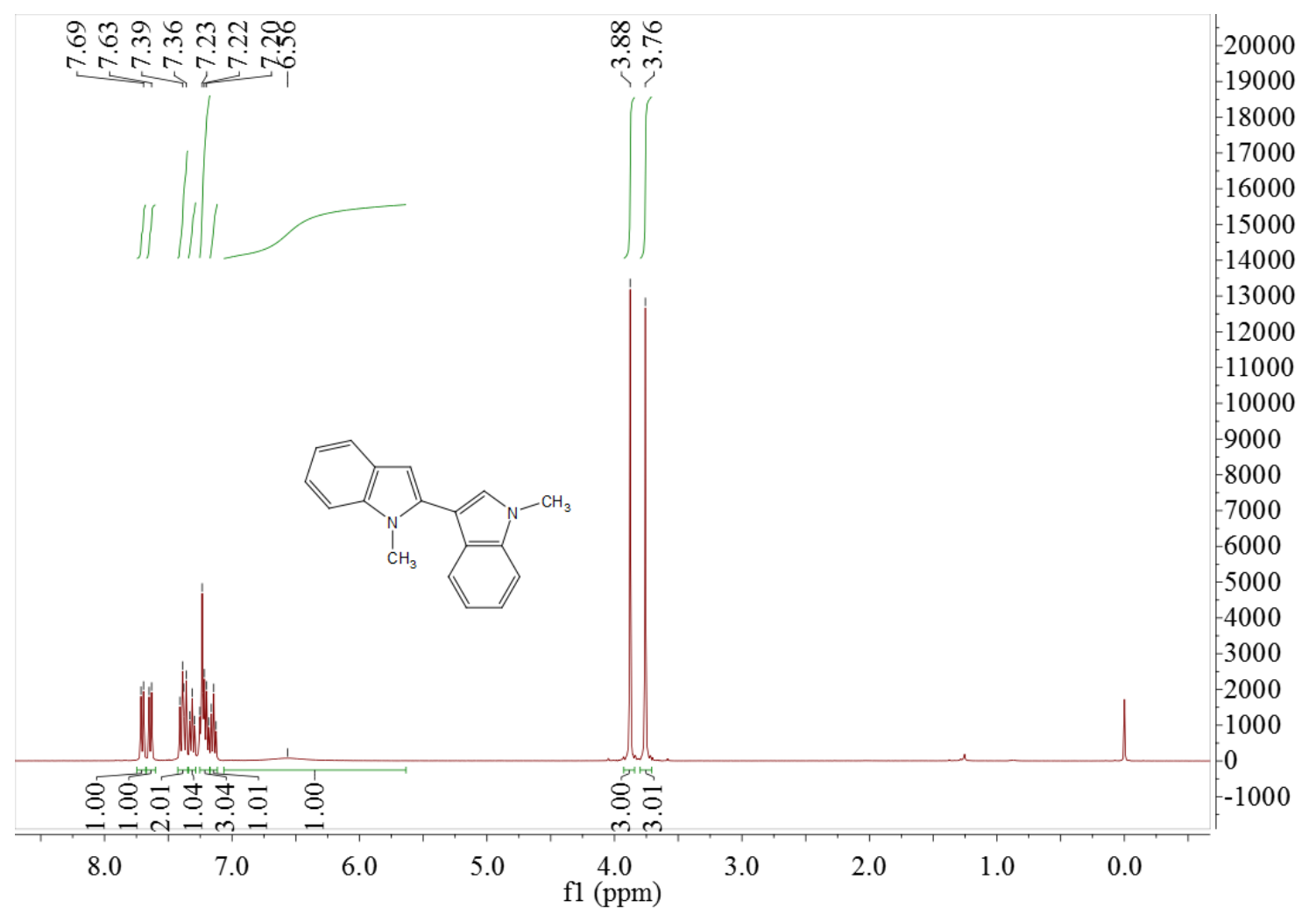

${ }^{1} \mathrm{H}$ NMR spectrum of 1,1'-dimethyl-2,3'-bi-1H-indole (8b)

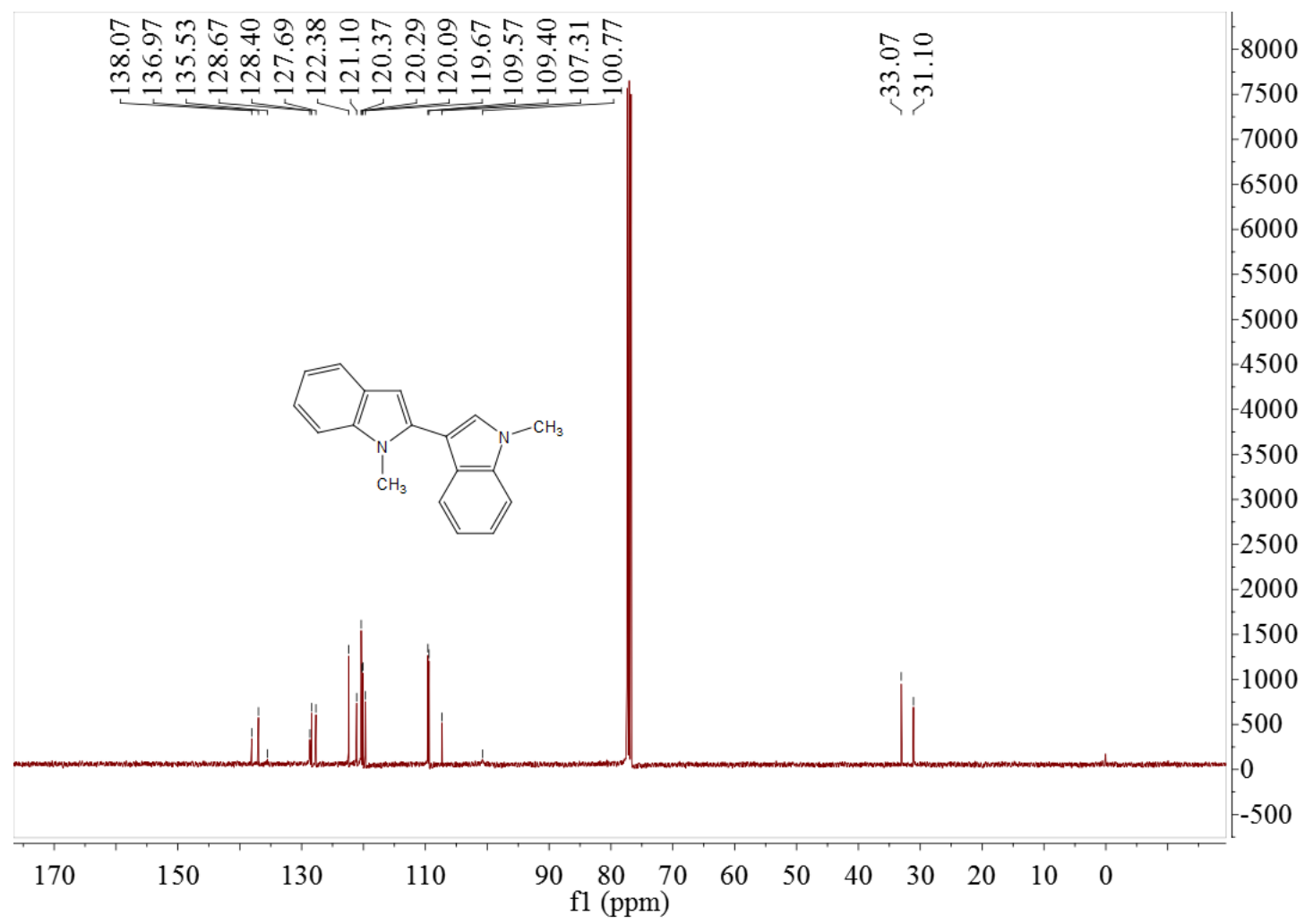

${ }^{13} \mathrm{C}$ NMR spectrum of 1,1'-dimethyl-2,3'-bi-1H-indole (8b) 


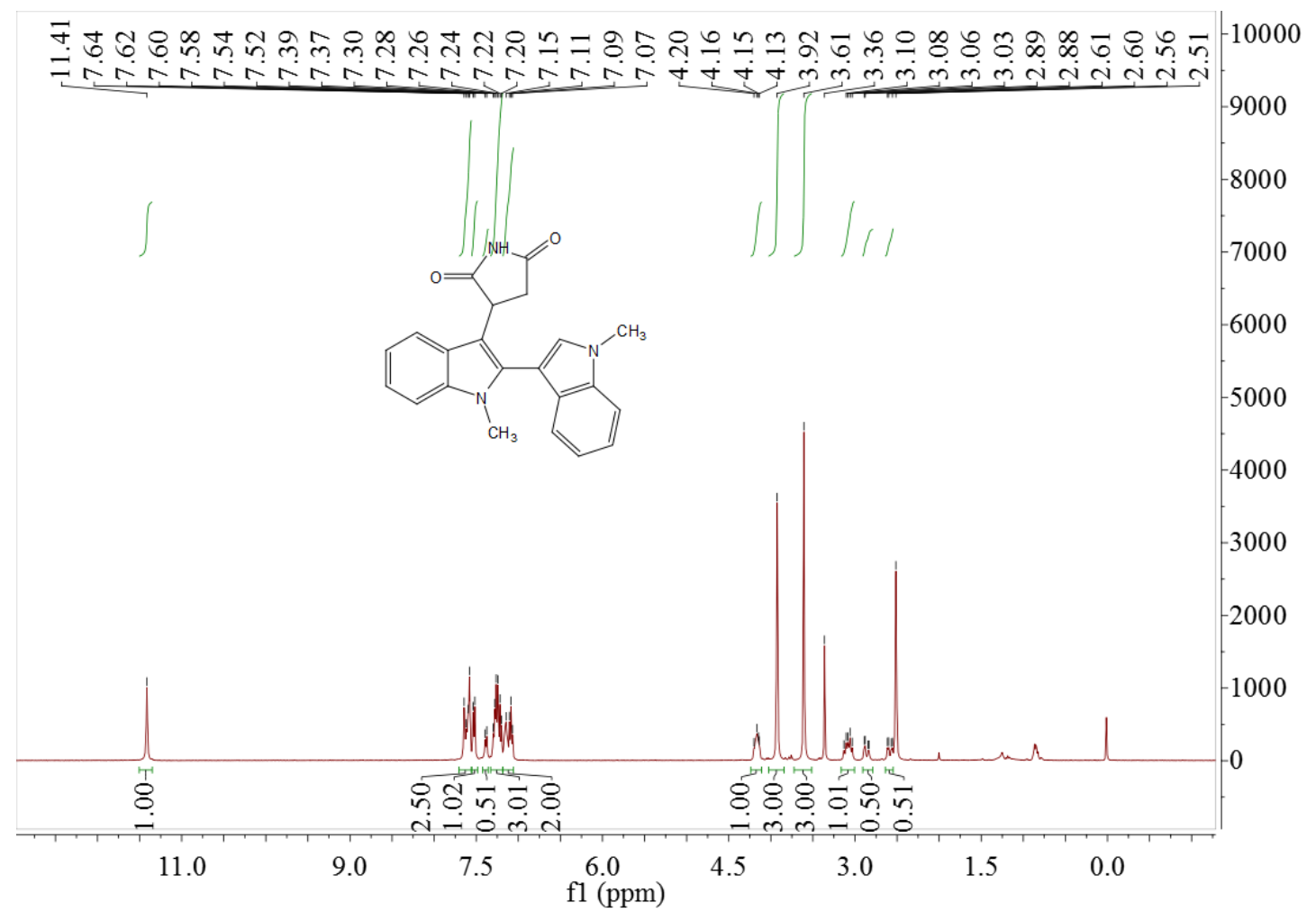

${ }^{1}$ H NMR spectrum of 3-(1,1'-dimethyl-2,3'-bi-1H-indolyl-3-yl)- pyrrolidine-2,5-dione (9a)

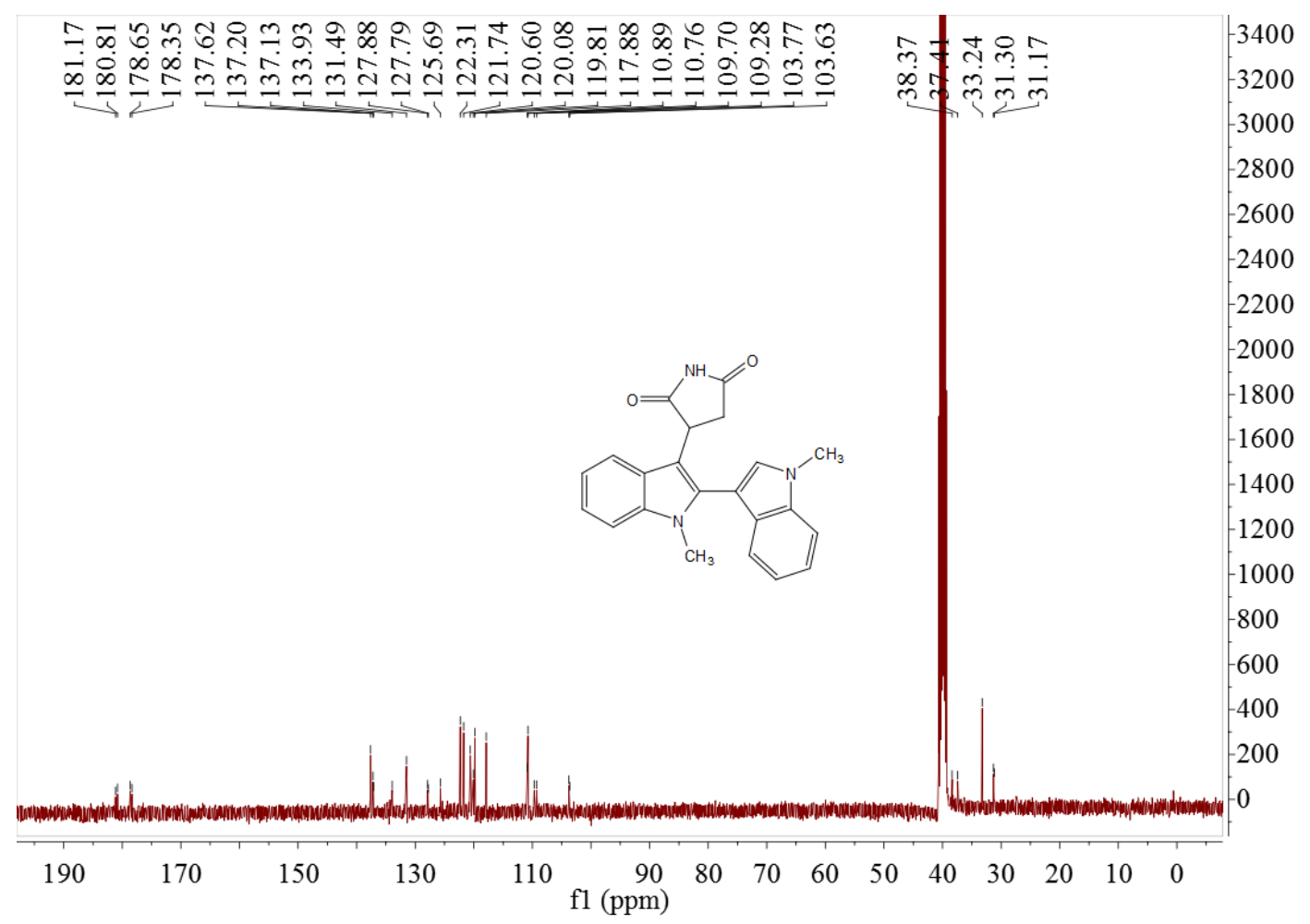

${ }^{13} \mathrm{C}$ NMR spectrum of 3-(1,1'-dimethyl-2,3'-bi-1 $H$-indolyl-3-yl)-pyrrolidine-2,5-dione (9a) 


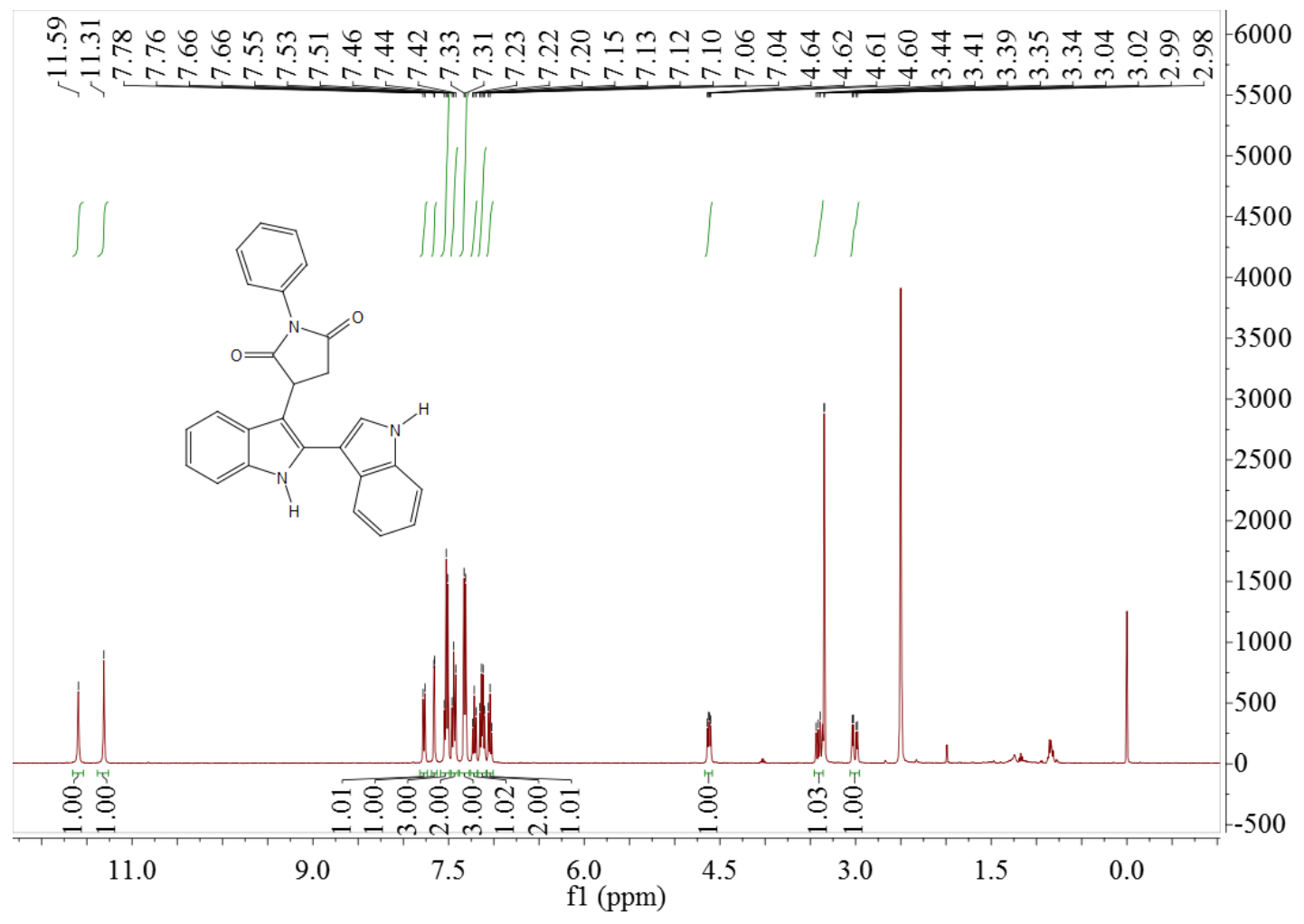

${ }^{1} \mathrm{H}$ NMR spectrum of 1-phenyl-3-(2,3'-bi-1H-indolyl-3-yl)-pyrrolidine-2,5-dione (9b)

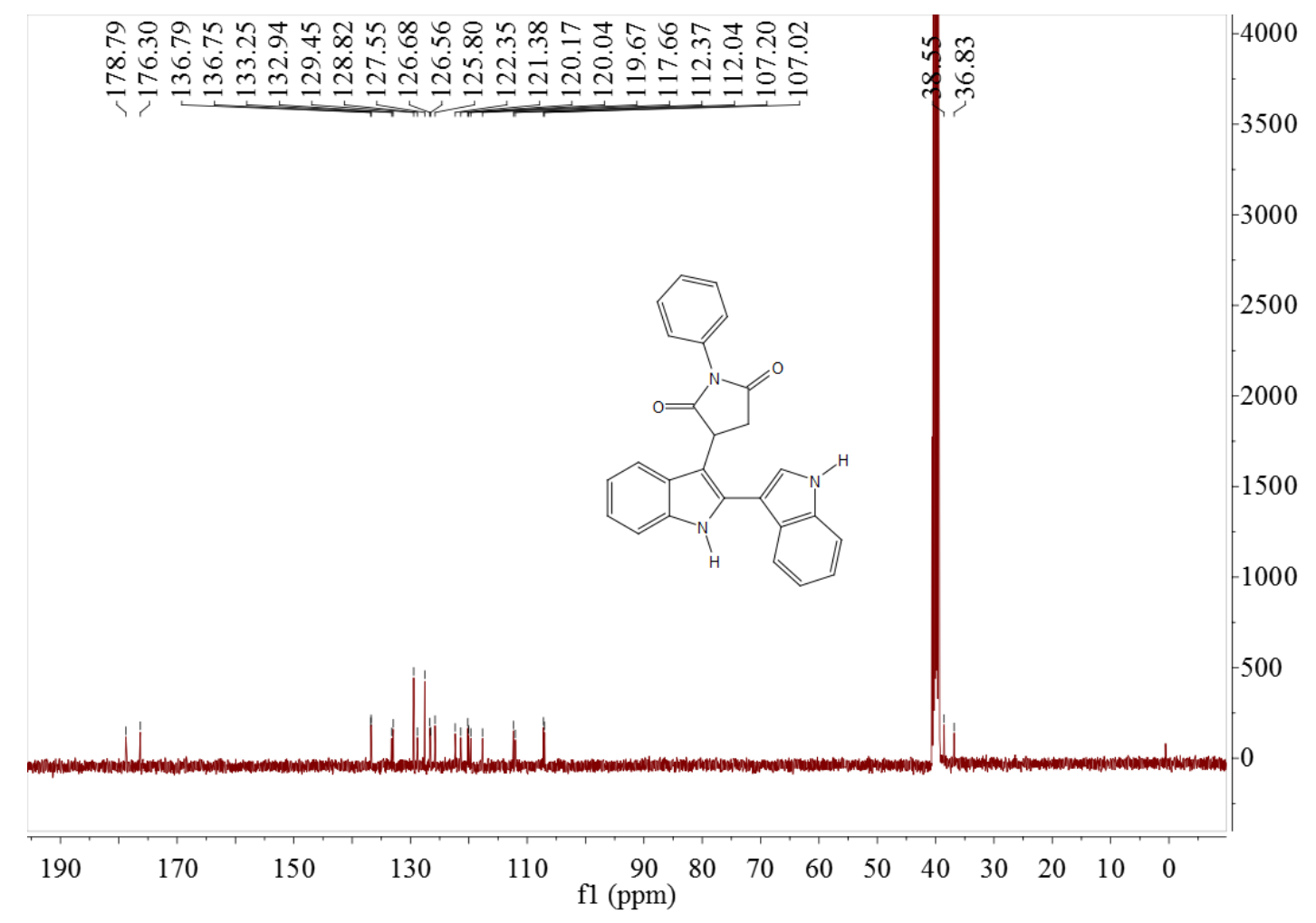

${ }^{13}$ C NMR spectrum of 1-phenyl-3-(2,3'-bi-1H-indolyl-3-yl)-pyrrolidine-2,5-dione (9b) 